\title{
Volume 6, Number 3
}

\section{Hipatia Press}

www.hipatiapress.com

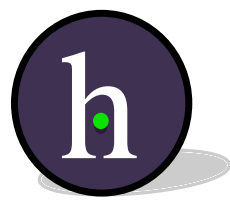

Ways of Masculinity in Online Dating Profiles: The Cases of Meetic.es

Articles

Masculinidades y Emociones como Construcciones Socioculturales: Una Revisión Bibliométrica - Juan Carlos Ramírez Rodríguez, María del Pilar Gómez González, Norma Celina Gutiérrez de la Torre, Marcela Viridiana Sucilla Rodríguez

Analysing Masculinity from the Key Theoretical Lenses and Searching for Linkages with Violence against Women- Anisur Rahman Khan \& Shahriar Khandaker

Masculinity and Rhythmic Gymnastics. An Exploration on the Transgression of Gender Order in Sport- Joaquín Piedra 288

Misogyny Online. A Short (and Brutish) History - Beatriz Villarejo....304 


\section{Hipatia Press \\ www.hipatiapress.com}

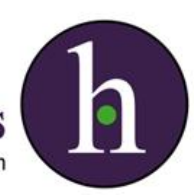

Instructions for authors, subscriptions and further details:

http://mcs.hipatiapress.com

Ways of Masculinity in Online Dating Profiles: The Cases of Meetic.es and AdoptaUnTio.es

Eva Espinar Ruiz'

Ismael Ocampo ${ }^{1}$

1) University of Alicante, Spain

Date of publication: October $21^{\text {st }}, 2017$

Edition period: October 2017 - June 2018

To cite this article: Espinar-Ruiz, E. \& Ocampo, I. (2017). Ways of

Masculinity in Online Dating Profiles: the cases of Meetic.es and

AdoptaUnTio.es. Masculinities and Social Change,6(3),196-216

To link this article: http://doi.org/10.17583/MCS.2017.2709

PLEASE SCROLL DOWN FOR ARTICLE

The terms and conditions of use are related to the Open Journal System and to Creative Commons Attribution License (CC-BY). 
MCS - Masculinities and Social Change Vol. 6 No. 3 October

2017 pp.196-216

\section{Ways of Masculinity in Online Dating Profiles: The cases of Meetic.es and AdoptaUnTio.es}

Eva Espinar Ruiz

University of Alicante
Ismael Ocampo

University of Alicante

\section{Abstract}

The analysis of masculinity has been a topic of growing interest in recent decades. Its study has incorporated a wide and diverse range of research areas and themes, including the representation of gender relations and identities on the Internet. Specifically, this article concerns the research area related to online dating websites and aims to compare the principal current tendencies related to identity -as provided by research on masculinity- with the way that men present themselves on two Spanish dating websites: Meetic.es and AdoptaUnTio.es. These types of virtual spaces have specific characteristics that facilitate the analysis of the masculine ideal among their users; or at least the characteristics that these men consider attractive to women. This research was carried out through a qualitative analysis supported by Atlas-ti. The principal results highlight the presence of traces of the so called egalitarian masculinity within predominant forms of traditional masculinity, characterized by a minimal process of reflection and introspection on the part of users of these websites.

Keywords: dating websites, masculinity, Internet, gender, qualitative analysis 


\section{Formas de Masculinidad en los Perfiles de las Páginas Web de Citas: Los Casos de Meetic.es y AdoptaUnTio}

Eva Espinar Ruiz

Universidad de Alicante
Ismael Ocampo

Universidad de Alicante

\section{Resumen}

El estudio de las masculinidades ha sido objeto de interés creciente en las últimas décadas. Su análisis ha ido abarcando una amplia diversidad de temáticas, incluyendo, entre las mismas, la representación en Internet de identidades y relaciones de género. Para este artículo se ha tomado como ámbito específico de investigación el constituido por las páginas web de citas. De esta forma, se han contrastado las principales tendencias en materia de identidad que destacan las aportaciones desarrolladas en el campo de estudio de las masculinidades con las presentaciones que hacen de sí mismos una muestra de hombres usuarios de dos páginas web de citas españolas: Meetic.es y AdoptaUnTio.es. Este tipo de espacios virtuales tiene particularidades específicas que facilitan el análisis del ideal de masculinidad de sus usuarios o, al menos, de las características que éstos consideran que resultan atractivas para las mujeres. La investigación se ha desarrollado a través de un análisis cualitativo, apoyado en el programa Atlas-ti. Entre los principales resultados, destaca la presencia de algunos rasgos relativos a las denominadas masculinidades igualitarias, dentro del predominio de formas tradicionales de masculinidad, caracterizadas por un mínimo proceso de reflexión e introspección por parte de los usuarios.

Palabras clave: páginas web de citas, masculinidades, Internet, género, análisis cualitativo. 


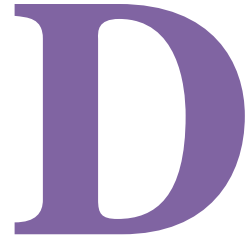

ating websites have grown in terms of the number of users and prestige in recent years, accompanied by the popularization of virtual social networks (Fullick, 2013). As of today, there are numerous online dating websites around the world that have become more diversified and specialized based on the preferences of their millions of users. While it is difficult to find reliable data on the number of people who use these websites, various studies have highlighted their continuous growth in terms of popularity (Ellison et al., 2006; Rosenfeld \& Thomas, 2012). Furthermore, online dating websites have lost much of the stigma they had in the past and are now widely used by a diverse group of people (Finkel et al., 2012).

Users of these websites are usually asked to fill out a brief personal profile. To produce this profile, users must engage in some kind of introspective work (Illouz, 2006) and at the same time design a strategy for integrating the qualities they consider most effective for finding a partner. This article focuses on the study of masculine identities as reflected in these profiles, taking as a sample an all-male group of users (seeking a female partner) from the websites Meetic.es and AdoptaUnTio.es. The strategies they use in their profiles permit the analysis of the ideal characteristics of contemporary men, or at least of those characteristics (emotions, attitudes, behaviors, hobbies etc.) that they consider will be most attractive to women1. In order to provide a foundation for this analysis, we present a summary of the theoretical framework and state of the art, which permits defining the principal characteristics of the tendencies and changes in contemporary masculinities. These tendencies will be compared with the profile strategies employed by users of the websites in question through a qualitative analysis.

\section{The study of masculinities}

The systematic analysis of the construction and representation of masculine identitiesis relatively recent and less common than the study of women's issues (Guasch, 2008). Somehow, the general androcentric nature of western culture and science has made gender invisible for men, in the same way that race is invisible for whites (Kimmel, 1993). As Gutmann explains 
(1998, p.82)), "masculinity is either ignored or considered to be the norm, such that (...) gender frequently refers to women and not to men". It wasn't until the end of the 1970's that the specific study of masculinity began, originating the so called men's studies (Carabí, 2000). This research field takes a constructionist approach in which male behavior is analyzed as socially and culturally attributed (Guttman, 1998).

Men's studies have been developed principally in two research areas: on one hand there is the analysis of traditional masculinity, linked to power and domination, and on the other there is the study of changes and new forms of masculinity in postmodern societies (Portell \& Pulido, 2012). In the first area, Pierre Bourdieu carried out an extensive analysis of masculine domination that posits that "the power of the masculine order manifests itself in the fact that it needs no justification: the androcentric vision is imposed as neutral and doesn't feel the need to gain legitimacy through discourse" (Bourdieu, 2000, p 22). This affirmation can be related to his concept of habitus, explained as the way in which individuals naturalize certain aspects and conducts of society, in this case the situation of supremacy of men over women. From this perspective, masculinity is usually said to be constructed based on the image of the other: the feminine (Subirats, 2007).

Masculine domination is not only exercised by men towards women but also among men themselves (Kauffman, 1999). In the 1980's Connell (1995) introduced the idea of hegemonic masculinity, adapting Gramsci's concept of hegemony to gender relations. For the author, hegemonic masculinity functions as a cultural reference of what a man should be in a determined social context, reproducing the patriarchal structure of that context. This hegemonic masculinity functions as a framework of reference for men in terms of norms, attitudes and values that should be possessed to be considered men and to benefit from masculine privileges. While the hegemonic model exists, Connell poses the existence of other models that serve as counter-references. According to the author, gender structures are dynamic and contextual, and thus the hegemonic model is constantly absorbing values and aspects of non-hegemonic groups, in order to maintain the structure of patriarchal domination.

Norms associated with hegemonic masculinity usually indicate that men should be "important, active, autonomous, strong, powerful, rational, emotionally under control and heterosexual" (Coll-Planas \& Missé, 2015, 


\section{Espinar \& Ocampo - Ways of Masculinity in Online Dating}

p.416), combative, aggressive (Padrós, 2012), homophobic and sexist (Sanfélix, 2011). However, with the influence of feminisms and LGBT movements, these components of what it means to be a man have been polemicized and have resulted in changes in modern masculinities (Badinter, 1993). These transformations have been at the root of what diverse authors have proposed as the concept of "new masculinities" (Carabí \& Segarra, 2000). The perspectives employed are diverse, although they usually coincide in signaling the heterogeneous character of contemporary vis-à-vis traditional masculinities (Carabí, 2000). In this sense, it is possible to differentiate the development of two general tendencies: one linked to the logic of consumer societies, hedonism and the cult of the body, and another linked to calls for equality in gender relations (Subirats, 2007).

The first tendency is characterized by a process of objectification of the male body, in much the same way as has happened with the female body. Mira (2015) carried out an analysis using Mark Simpson's concept of the "Spornosexual male" about the overrepresentation of the physical-sexual attributes of men in the media, converting them into passive objects of pleasure. There does exist, in this way, a negative feminization of the masculine in pursuit of desire and specific canons of beauty, that provokes complexes and insecurities in men related to their bodies (Bordo, 1999). For some authors, this new model of masculinity is a product of marketing that -from an apparent image of gender equality- results in a process of asexualization in which both genders hold a single identity and personality directed towards consumption, making them into objects with market qualities (Hernandez Ochoa, 2011).

Meanwhile, the second tendency is related to the call for antihomophobic and anti-sexist values (O'Neil, 2015), with fewer emotional restrictions (Lomas, 2013), and with the development of new models of paternity and assignment of roles and responsibilities (Johansson \& Ottemo, 2013). These processes have been associated with terms such as positive masculinity (Duncanson, 2015) or inclusive masculinity (Anderson, 2008). Duncanson (2015) suggests that positive masculinity is an adaptation of the hegemonic model that seeks to maintain masculine supremacy and predominance in society, but despite this, it supposes a transitory stage within the process of eradication of gender hierarchies, 
forming what the author calls hybrid masculinities. On the other hand, Anderson (2008) observes that highly educated and middle class men have friendships with homosexual men and with women, breaking with certain classic taboos in which men could not have friendships with women and also belittled homosexuality. Peacock and Barker (2014) point out that many men have begun to understand that sexist behavior is counterproductive for them, which is promoting the appearance of organizations and men's groups around the world that advocate for new forms of masculinity. Furthermore, Lomas (2013) raises that it is possible that many of the elements of positive masculinities are in some contexts already part of hegemonic masculinity.

\section{Online dating: state of the art}

Sociological interest in the topic of online dating sites is -necessarilyrecent, as is the popularization of these websites. However, there are already a good number of related studies, carried out in the last decade, that focus on different themes: the construction and representation of identities, the characteristics and preferences of site users, infidelity, the efficiency of these sites, pages for specific user groups, and differences between intimate relationships online and offline, etc.(Casemiro, 2014).

The analysis of identity representation is usually centered on the study of self-presentation in the profiles that users must construct, with two particularities of dating websites with respect to the rest of social media sites: the objective of these profiles and the way in which they are created (Illouz, 2006). The objective is usually to establish an offline encounter, which somewhat limits the possibility of showing an identity that differs from the reality. On the other hand, Illouz (2006) posits that the questionnaires that users fill out are created by experts and they are designed to reach an acceptable level of veracity. In this way these profiles of the self presumably suppose a task of introspection on the part of users (Gonzalez García, 2010).

According to Erving Goffman (2008), in general, when getting involved with each other, individuals try to give the best impression, and as such they present an idealized version of themselves. This idealized self is usually made up of the characteristics that are most accepted and valued in the corresponding context. Illouz (2006) claims that the users of online 


\section{Espinar \& Ocampo - Ways of Masculinity in Online Dating}

websites not only present an idealized version of who they are or would like to be, they also do so in a generic way, given that the desired recipient is not a single individual but rather a diverse group of people. For this reason, standardized presentation strategies are used that make profiles quite uniform. Furthermore, the author affirms that these online spaces convert the individual into an object for consumption within a structured and categorized market. Users must define themselves in a competitive framework, in which they will have a greater chance of success if they adapt to the dominant norms of conduct.

In terms of the analysis of users' preferences when choosing a partner via these websites, different researchers have tried to signal the attractiveness factors (Hitsch et al., 2010). Thus, although some studies have highlighted the role of photos and physical characteristics (Morgan et al., 2010; Bak, 2010; Chappetta \& Barth, 2016), other factors are also shown to be relevant such as personality (Morgan et al, 2010)), an authentic or warm nature that inspires confidence and appears extroverted in the case of men, or appearing feminine with high self-esteem and not narcissistic in the case of women (Fiore et al., 2008). The significant differences between the results obtained in different researches could be related to the groups selected to be studied and the specific online dating sites used as sources of information.

Other studies have focused on the evolution and influence of online dating sites. In this area, Barraket and Henry-Waring (2008) analyzed the impact these sites have on how people meet each other, through in-depth interviews with a sample of users. Similarly, Rosenfeld and Thomas (2012) studied the evolution of the way couples are formed in the United States from 1940 to 2010. Looking at the past 15 years of the studied period, they observed an increase in the number of couples that meet via the Internet, and they analyzed the way in which this tool serves as a substitute for more traditional ways of finding a mate. The work of Chaulet (2009) is also worth noting. He analyzed the perception of trust in online dating websites through in-depth interviews. The author concludes that, even though in the past these sites suffered from a negative stigma, with the passing of time they have gained confidence and respect in society.

In a similar way to the present article, some of these studies have specifically adopted a gender perspective. Thus, for example, Martínez- 
Lirola (2012) identified the communication strategies of male users of the social network Badoo.com, and found certain traditional elements, such as men taking the initiative and the expectation that women should be sweet and affectionate. Likewise, Marcus (2014) highlighted the representation of traditional gender stereotypes in which men showed themselves as hard workers and as active individuals with a passion for sport activities. Wang (2012) focused her attention on heterosexual users of a Thai dating website, and concluded that the online dating space permitted the representation of non-hegemonic models of masculinity, with the co-existence of multiple identities. These last conclusions are related to those obtained by Chappeta and Barth (2016), who found in a research on American students that users preferred profiles that did not conform to traditional gender stereotypes. However, the students in the sample were mostly interested in sporadic or short-term relationships. Along these lines, Walker and Eller (2016) carried out a comparative study of the profiles of heterosexual and homosexual men, concluding that there was a greater presence of traditional masculine discourse among homosexual men. Finally, Casemiro (2014) studied how identities were constructed on the website Meetic.com, observing the differences among men and women. The author found that men were more rational and practical in their representations than women, who were more emotional, reproducing traditional gender stereotypes.

\section{Methodological notes}

\section{The selected websites}

The sources of the secondary data for this research are the dating websites Meetic.es and AdoptaUnTio.es (AUT). These two webpages were chosen due to their popularity in Spain, their different models, and their orientation towards different publics. AUT is directed to young people, and Meetic, although focusing on a wide spectrum, takes a more serious approach that attracts older users.

Of French origin, Meetic was created in 2001 and claims (on its own website) to be a leader in the sector in Europe2. AUT also originated in France and was created in 2008. In 2013 it expanded into the Spanish market and after a year had achieved a following of some 500,000 registered users. On both webpages 3 users must create a profile that 


\section{Espinar \& Ocampo - Ways of Masculinity in Online Dating}

highlights a main photo, together with smaller optional photos that the user can add. The rest of the elements of the profile are: name, age, physical characteristics and a description of one's personality and interests. The search for other users occurs through a search function in which age and geographic preferences can be selected. Other criteria that can also be added include physical appearance, interests and sociodemographic data.

\section{Population and sample selection}

The research population includes the self-presentation of the profiles of men looking for a female partner on the two aforementioned webpages. However, the geographic origin of users was limited to the area within a 50 $\mathrm{km}$ radius of the city of Alicante (Spain). This strategy was an attempt to provide for cultural and demographic similarities in the sample, in order to avoid, whenever possible, unidentified factors that could distort the results.

Given the qualitative approach of the study, we opted for sequential, non-probabilistic sampling, in which final cases were selected according to saturation criteria. The specific selection procedure was as follows:

1. Identification, through the search function of each website, of men attempting to contact women and who resided in Alicante or within a 50 $\mathrm{km}$ radius of the city.

2. The first 20 profiles that appeared on each website were selected.

3. The procedure was repeated on three different days: April 12, 13 and 20 of 2015. Through these three sessions, 120 profiles were collected.

4. Profiles were eliminated that were incomplete, or included an exceedingly brief or confusing description. This was the case for users that defined themselves only through several meaningless words such as: "The price doesn't matter as long as it's worth it" or "Legalize it".

After the first reading, analysis and comparison of the collected material, it was observed that it would be possible to achieve saturation of information with around 30 profiles per webpage. Thus, the definitive sample is made up of a total of 60 profiles. The ages of the users that make up the sample range from 21 to 64 years, with an average of 43 years for Meetic.es and 30 years for AUT. 


\section{Data analysis and presentation of results}

A thematic qualitative analysis (Braun \& Clarke, 2006) was carried out of the self-presentations from the profiles of the 60 users selected for the sample. Atlas.ti software was used for support, to facilitate the process of coding the information. The coding process joined codes derived from the theoretical framework and inductive codes derived from the direct reading of the material (Valles, 2000).

In this sense, the aim of the analysis was not to quantify or make statistical inferences but rather to search for elements that could describe aspects of masculine identities contained within the sample. For this reason, it was decided that the research would only be carried out considering the texts, leaving aside the analysis of the photos, given the need to identify the process of self-reflection that the users supposedly have to carry out in using these online spaces.

The presentation of results here is organized according to the principal thematic areas detected, inserting phrases and expressions of the users. These text quotations are identified by indicating their webpage of origin (AUT or Meetic). Finally, results are discussed in terms of the related theoretical areas and the objectives of the study, leaving proposed future lines of research for the conclusions.

\section{Results}

After coding and comparing the texts of the profiles, it can be observed that they are usually constructed based on three axes: (1) description of the personality; (2) hobbies and interests; (3) qualities sought in a potential partner. The three components can appear interrelated in a single profile, and it is not always easy to differentiate them. In any case, these are the elements that structure the different presentations, as was also concluded in a study by Walker and Eller (2016) ${ }^{4}$.

\section{Personality: sensitive or adventurous guys}

Among all the analyzed profiles, only a little over half mentioned personality characteristics. This seems to reflect the lack of introspective 


\section{Espinar \& Ocampo - Ways of Masculinity in Online Dating}

work on the part of a large portion of the sample. Furthermore, it is possible to speak of a generalized difficulty in terms of detailing the traits of one's own personality; indeed, some of the users did not even attempt it: "it is difficult to define oneself, it's true, I am a normal person, I think" (AUT-6). Among those who did, the majority opted for synthetic and brief formulas. In fact, the users often employed a very limited diversity of terms, which can be grouped into two categories:

A) Those adjectives that indicate a sensitive, affectionate or friendly character of the users: "friendly, joyful, intuitive, big hearted, accessible, hard worker, attentive" (AUT-25). Traits that could be related to a model of masculinity that is cordial and friendly.

B) Those other terms that show users as independent, adventurous and as entrepreneurs: "a guy with clear ideas, extroverted and adventurous, with a restlessness to try out new and surprising sensations" (AUT-23); "adventurous, crazy, talkative, daring..." (AUT-27). This adventurous spirit also appears when users describe their hobbies and interests.

Finally, the frequent use of the term "guy" should be highlighted when users present themselves in their profiles (compared to the infrequent use of other terms such as "man" or "person"): "I'm a guy who is separated, I don't have children, I am extroverted..." (Meetic-12). This formula seems to respond to the informal logic of this type of webpage. At the same time, it could be considered as a strategy that permits users to transmit a young character that is modern and relaxed.

\section{Hobbies and interests: "living life"}

A large part of the users opted to highlight their hobbies and interests as principal components of their profiles. In this sense, the mention of sport activities linked to an active and non-sedentary lifestyle is frequent: "I love sports, the beach, the unknown... the adventure of living life!!" (Meetic-1) "I like to practice sports. I'm a happy guy, I really like to travel" (AUT-19). Another area that appears frequently is linked to enjoyment of the social scene. Activities such as going out for a drink, having good conversations, being with friends or proclaiming the importance of friendship, are 
elements that many users highlight: "I'm a normal guy that likes to go out, be with friends" (Meetic-25). These characteristics show the user as open, friendly and extroverted. It is also frequent to mention interest in film, music or literature, that in addition to being forms of entertainment can demonstrate the user's interest in artistic and cultural issues: "I'm extroverted, friend of my friends, I like the cinema, taking a walk, going to dinner, travelling..." (Meetic-12) or "I like to write, I love reading, the cinema, travelling, water and winter sports, nature and the sea" (Meetic-17). These examples show the wide range of hobbies that some users include in their profiles and that define them as individuals that are active, extroverted and with certain cultural interests.

Just as in the case of the description of one's personality, many of the profiles opt for brief texts that do not go into detail in terms of explaining hobbies and interests. In this sense, the minimal presence of activities related to the work or academic environment, home and family is worth noting, as they do not seem to be aspects valued as important in finding a partner on these webpages.

\section{The sought partner: looking to share and have fun}

In the majority of the profiles analyzed, there are allusions and comments about the type of relationship that is being sought and about the main characteristics of the women that these men want to contact. In this area, two themes can be differentiated: A) what is being sought in the potential partner; and B) the type of relationship they want to establish.

\section{The ideal girl: sincere or fun-loving}

When describing the woman with whom they want to make contact, the users primarily mention personality characteristics. Specifically, the presence of two semantic camps is worth highlighting. On one hand, being happy, friendly and fun-loving: "I guess I am looking for what everyone is looking for: someone who I can laugh with" (AUT-19); on the other hand, sincerity: "I like it when things are clear, and I like sincerity" (AUT-28). Significant differences between the two webpages can be observed in the description of these preferences: while users of AUT usually want a woman 
with a fun-loving character, in Meetic.es the option of sincerity is more widespread.

Other traits, such as age or physical appearance are barely mentioned by the users: "I'm looking for a good person, attractive if possible..." (Meetic15 ) or "If you are more or less attractive, between 48 and 57 years old..." (Meetic-30). Also little mentioned is the presence of characteristics such as being romantic, affectionate, passionate or independent, "authentic" and "with personality". Furthermore, when trying to describe their ideal woman, users usually draw parallels with the traits they have used to describe themselves. They employ similar attributes, showing their preference for someone like them.

\section{Type of relationship: serious or informal}

In coherence with what has been shown in previous sections, reflections about the ideal couple relationship are not extensive. Furthermore, the type of relationship is mentioned less than are women's personality characteristics. Even so, some relevant aspects can be highlighted. In this sense, the mention of the desire to share something with a possible partner is frequent among users: "I would like to find someone with whom to share, and enrich each other mutually" (Meetic-17). This type of commentary is more common on Meetic.es than on AUT and encompasses different possibilities that range from sharing "moments" (Someone with whom to share good moments" - Meetic-23) to "life" ("I'm a simple person looking for someone with whom to share my life" - Meetic-26). These expressions are the closest declarations of preferences for egalitarian sentimental relationships, although we have not detected the presence of comments explicitly related to this type of relationship.

Finally, it is on Meetic.es where a greater priority is shown for commitment and for serious and stable relationships: "I dream of sharing good times in a stable way with someone special" (Meetic-8). The explicit allusion to casual relationships is practically inexistent on both webpages, even if on AUT users avoid references to formal relationships and opt for a non-committal discourse: "I'm not looking for anything in particular. I've been in Alicante for a short time and I'm looking to meet people and get to 
know the city. Any ideas are welcome, whether it's sports or social. I adapt to it all!" (AUT-29).

\section{Discussion}

The analysis of the selected sample shows a lack of reflection on the part of the users in terms of describing their own personalities. There is a clear preference for presenting themselves in a synthetic and repetitive way, instead of going into more detail and being original. This confirms what was described by Illouz (2006), that online dating spaces do not promote processes of reflection on the part of the subjects, who prefer to follow standardized forms of self-presentation. However, this could be related at the same time to characteristics of traditional masculinity, linked with a supposed limitation of men when it comes to expressing feelings or showing personal issues. This could also be interpreted as a strategy that users tend to develop, based more on the description of what they "do" as opposed to who they "are". In this sense they opt for an active masculine identity exemplified in characteristics like being extroverted and adventurous, that Marcus (2014) also found in the results of her study.

The limited diversity of our sample seems to contrast with the results obtained by Wang (2012), who identified the presence of a plurality of forms of masculinity. Thus, our study allows the grouping of most of the profiles into two categories: sensitive guys and adventurous guys. Both groups share traits that could be associated with traditional models of masculinity (primarily the apparent incapacity to express oneself in terms of identities and feelings). Despite this, there are also aspects that can be related to the concept of hybrid identities, in agreement with what has been suggested -in other areas- by authors such as Duncanson (2015). In this sense, the absence of aggressiveness and sexism, traditionally linked to masculine stereotypes, should be highlighted. Also, the direct mention of socioeconomic and work status is omitted among users, an aspect that contradicts the importance of these areas in other studies (Marcus, 2014; Walker \& Eller, 2016). On the contrary, the profiles of various users show them as relatively open emotionally, with a preference for relationships based on friendship ties and the desire to share different dimensions of daily life with a woman. These results could reflect what is suggested by Lomas (2013) in terms of the fact that in determined contexts the hegemonic model of masculinity excludes features of the discursive forms 
of traditional masculinity, giving rise to more fraternal identity styles. Certainly, we can see a relative adoption of some characteristics of the so called positive masculinities, although this is reflected primarily in the absence of several elements related to traditional masculinity and not so much in the explicit reference to issues such as equality between men and women.

On the other hand, users seem to shy away from referring to their bodies, both their own and those of their potential partner, which apparently contradicts prior studies that pointed out the importance of physical appearance in online dating, especially in the case of men (Morgan et al. 2010; Bak 2010). Thus, the fact that users do not describe their own physical appearance requires further study. It could be a strategy that they consider to be appropriate in the search for a female partner and, as such, it might not mean that they give no importance to their physical appearance. An analysis of the photographs could provide more information on this subject. For the moment, based on our data, we see the absence of elements related to the hedonistic tendency of masculinity that some authors have highlighted (Subirats, 2007) and that we pointed out in the theoretical framework.

Certainly, the users of our sample construct profiles formed by hybrid masculine identities. This hybrid takes on mixed forms of masculinity that bring together aspects of traditional masculinity -resistance to showing emotions, self-definition based on what they do as opposed to who they are, and preference for identification with active forms of masculinity-, with elements linked to egalitarian models of masculinity, without directly expressing their related values and premises. This hybrid of masculinities could be related to what was suggested by Connell (1995), in terms of the hegemonic model being in a constant process of change, with a dynamic structure that feeds itself with external and contradictory inputs. In this sense, we can concur with Duncanson's (2015) criticism of so called positive masculinity, by showing a model of being a man that has lost the traditional discourse but that still maintains many of its characteristics.

\section{Conclusions and future areas of study}


The analysis of our sample shows a group of men with apparent difficulties in engaging in a process of self-reflection about their own personalities. The majority of the online dating users opt to construct their profiles in a brief way with a synthetic format in which they mention their hobbies, preferences and activities, showing little introspection. This lack of reflection could be related to traits that are characteristic of traditional masculinity, but it could also be -as suggested by Illouz (2006) - related to the format of the dating websites. In conclusion, these aspects require further study both in terms of comparative research-using samples of men and women- and qualitative inquiries - interviewing users with the aim of understanding and interpreting the strategies that are put into practice in these virtual spaces. In fact, a comparative research involving the actors' point of view, would allow to overcome the static perspective of the present investigation, and to adopt a relational and dynamic approach.

Among the profiles analyzed, there is a combination of characteristics from the different tendencies in current masculinities described by prior authors. This hybrid nature presents certain elements of homogeneity (such as the schematic and brief structure of the profiles) and a relative heterogeneity marked by the different traits, hobbies and interests of the users. Thus, we can differentiate a group of profiles that adopt sensitive and fraternal models of masculinity and that can be related to the tendencies of positive masculinity. Meanwhile, other profile strategies focus on elements of leisure, physical activity and adventure which permits linking the users with certain elements both of traditional and hedonistic masculinity, albeit without direct references to physical appearance. The analysis of the presence of hedonistic tendencies could benefit from future research that includes photographic material in addition to the study -through interviewsof the authors' interpretations of their own profiles.

\section{Notes}

${ }^{1}$ In this regard, the article does not propose a research on online masculine identities, but simply the information available through online dating websites has been considered as a valuable material for an approach to the analysis of contemporary masculinities. Obviously, the analyzed sample is not representative of the general population, but it can still allow the study of those features that male users of an increasingly socially accepted virtual space deploy in the attempt to find a female partner.

2 https://www.meetic.es [accessed on 29/11/2016]. 
3 https://www.adoptauntio.es/ [accessed on 29/11/2016]

4. Walker and Eller (2016) identified a fourth axis, with the inclusion of the professional dimension. In our study, however, economic and work-related aspects are not explicitly mentioned.

\section{References}

Anderson, E. (2008). Inclusive masculinity in a fraternal setting. Men and masculinities, 10, 604-620

Badinter, E., (1993). XY: la identidad masculina. Madrid. Alianza editorial

Bak, P. (2010). Sex Differences in the Attractiveness Halo Effect in the Online Dating Environment. Journal of Business and Media Psychology, 1, 1-7. Retrieved from http://journal-bmp.de/wpcontent/uploads/2010/11/JBMP-1-2010-Halo-Bak.pdf

Barraket, J. \& Henry-Waring, M. S. (2008). Getting it on (line) Sociological perspectives on e-dating. Journal of Sociology, 44(2), 149-165. doi: https://doi.org/10.1177/1440783308089167

Bordo, S. (1999). The Male Body: A New Look at Men in Public and in Private. Nueva York: Farrar, Straus and Giroux.

Bourdieu, P. (2000). La dominación masculina. Buenos Aires: Anagrama. Braun V. \& Clarke V. (2006). Using thematic analysis in psychology. Qualitative Research in Psychology, 3(2), 77-101. Retrieved from http://eprints.uwe.ac.uk/11735

Carabí, À. (2000). Construyendo nuevas masculinidades: una introducción. In À. Carabi \& M. Segarra (Eds.), Nuevas masculinidades (pp.1527). Barcelona: Icaria.

Carabi, À. \& M. Segarra (Eds.) (2000). Nuevas masculinidades. Barcelona: Icaria.

Casemiro, C. (2014). Portuguese online dating: Explorando las diferencias de género en la presentación personal. Revista Teknokultura, 11 (1), 117-141.

Retrieved

from

http://teknokultura.net/index.php/tk/pages/view/opr-170

Chappetta, K., \&, Barth, J. M. (2016). How gender role stereotypes affect attraction in an online dating scenario. Computers in human behavior, 63, 738-746. doi:

https://doi.org/10.1016/j.chb.2016.06.006 
Chaulet, J. (2009). La construcción equipada del vínculo amoroso. Las webs de encuentros y sus «caminos de confianza». Redes: revista hispana para el análisis de redes sociales, 16(4), 91-127. doi: https://doi.org/10.5565/rev/redes.366

Coll-Planas, G. \& Missé, M. (2015). "Me gustaría ser militar". Reproducción de la masculinidad hegemónica en la patologización de la transexualidad. Prisma social: Revista de investigación social, 13, 407-432. Retrieved from http://www.isdfundacion.org/publicaciones/revista/numeros/13/secci ones/tematica/t_12_masculinidad_transexualidad.html

Connell, R.W, (1995). Masculinidades. México D.F: Universidad Nacional Autónoma de México, Programa Universitario de Estudios de Género.

Duncanson. C. (2015). Hegemonic masculinity and the possibility of change in gender relationship. Men and masculinities, 18 (2), 1-18. doi: https://doi.org/10.1177/1097184X15584912

Ellison, D., Heino, R., \& Gibbs, J. (2006). Managing Impressions Online: Self-Presentation Processes in the Online Dating Environment. Journal of Computer Mediated Communication, 11(2), 415-441. doi: 10.1111/j.1083-6101.2006.00020.x

Finkel, E.J., Eastwick, P.W., Karney, B. R., Reis, H. T., \& Sprecher, S. (2012). Online dating: A critical analysis from the perspective of psychological science. Psychological Science in the Public Interest, 13, 3- 66. doi: 10.1177/1529100612436522

Fiore, A.T., Taylor, L.S., Mendelsohn, G.A. \& Hearst, M. (2008). Assessing attractiveness in online dating profiles. In Proceedings of the twenty-sixth annual SIGCHI conference on Human Factors in Computing Systems (pp. 797-806). ACM. Retrieved from https://www.ischool.berkeley.edu/research/publications/2008/assessi ng-attractiveness-online-dating-profiles

Fullick, M. (2013) "Gendering" the Self in Online Dating Discourse. Canadian Journal of Communication, 38(4), 545-562. doi: https://doi.org/10.22230/cjc.2013v38n4a2647

Goffman, E. (2008). La presentación de la persona en la vida cotidiana. Buenos Aires: Amorrortu.

González García, J. (2010). Atrapados en la red. Las nuevas redes románticas. Kairos: Revista de temas sociales, 14 (25) 1-16. 
214 Espinar \& Ocampo - Ways of Masculinity in Online Dating

Retrieved

from

https://dialnet.unirioja.es/descarga/articulo/3702324.pdf

Guasch, O. (2008). Los varones en perspectiva de género. Teorías y experiencias de discriminación. Asparkía. Investigació feminista, 19, 29-38. Retrieved from http://www.erevistes.uji.es/index.php/asparkia/article/view/467/386

Gutmann, M. C. (1998). Traficando con hombres: la antropología de la masculinidad. La ventana. 8, 47-99.

Hernández Ochoa, D. (2011). La "confluencia" de los géneros a través del sistema mediático: De la mujer sumisa y el macho ibérico al "ser andrógino". Papers: revista de sociología. 96(2), 569-587. doi: http://dx.doi.org/10.5565/rev/papers/v96n2.137

Hitsch, G. J., Hortaçsu, A. \& Ariely, D. (2010). What makes you click?Mate preferences in online dating. Quantitative marketing and Economics, 8(4), 393-427. doi:10.1007/s11129-010-9088-6

Illouz, E. (2006) Intimidades congeladas. Las emociones en el capitalismo. Madrid: Katz editores.

Johansson, T. \& Ottemo, A. (2013). Ruptures in hegemonic masculinity: the dialectic between ideology and utopia. Journal of gender studies, 24(2), 192-206. doi: http://dx.doi.org/10.1080/09589236.2013.812514

Kaufman, M. (1999). La construcción de la masculinidad y la triada de la violencia. In Violencia doméstica (pp. 52-69). Cuernava-Morelos (México): Cidhal, PRODEC, Centro de Documentación Betsie Hollants.

Kimmel, M. S. (1993). Invisible Masculinity. Society, 30(6), 28-35. Retrieved from https://www.sunypress.edu/pdf/61061.pdf

Lomas, T. (2013). Critical Positive Masculinity. Masculinity and social change, 2(1), 165-183. doi: http://dx.doi.org/10.4471/mcs.2013.28

Marcus, S. (2014). Online dating profile analysis: The intersection of identity, gender \& religion. Proceedings of the New York State Communication Association, 8, 1-15. Retrieved from http://docs.rwu.edu/nyscaproceedings/vol2013/iss2013/8

Martínez-Lirola, M. (2012). Aproximación a la interacción virtual: el caso de la red social Badoo. Palabra Clave, 15 (1), 107-127. doi: 10.5294/pacla.2012.15.1.5 
Mira, A. (2015). Mario Casas y el hombre "Depornosexual": La "espectacularización erótica del cuerpo masculino. Área abierta, 15(1), 15-30. $\quad$ Retrieved from http://revistas.ucm.es/index.php/ARAB/article/view/47601

Morgan, E. M., Richards, T.C., \& VanNess, E.M. (2010). Comparing narratives of personal and preferred partner characteristics in online dating advertisements. Computers in Human Behavior, 26(5), 883888. doi: https://doi.org/10.1016/j.chb.2010.02.002

O'Neill, R. (2015). Whiter critical masculinity studies? Notes on inclusive masculinity theory, postfeminism, and sexual politics. Men and masculinities, $\quad 18(1), \quad 100-120 . \quad$ doi: https://doi.org/10.1177/1097184X14553056

Padrós, M. (2012). Modelos de Atractivo Masculinos en la Adolescencia. Masculinities and Social Change, 1(2), 165-183. doi: http://dx.doi.org/10.4471/MCS.2012.10

Peacock, D., \& Barker, G. (2014). Working with men and boys to prevent gender-based violence: principles, lessons, learned, and ways forward. Men and masculinities, 17(5), 578-599. doi: https://doi.org/10.1177/1097184X14558240

Portell, D., \& Pulido, C. (2012). Communicative acts which promote new masculinities. Overcoming hegemonic masculinity in the workplace and school. Masculinities and Social Change, 1(1), 61-80. doi: http://dx.doi.org/10.4471/mcs.2012.04

Rosenfeld, M. J., \& Thomas, R. J. (2012). Searching for a mate the rise of the internet as a social intermediary. American Sociological Review, 77(4), 523-547. doi: https://doi.org/10.1177/0003122412448050

Sanfélix, J. (2011). Las nuevas masculinidades. Los hombres frente al cambio en las mujeres. Prisma Social: revista de ciencias sociales, 7 , 220-247. Retrieved from http://www.isdfundacion.org/publicaciones/revista/numeros/7/seccio nes/tematica/08-hombres-frente-cambio-mujeres.html

Subirats, M. (2007). Ser hombres. In M. Castells \& M. Subirats (Eds.), Mujeres y Hombres: ¿Un amor imposible? (pp.49-135). Madrid: Ed Alianza.

Valles, M. S. (2000). Técnicas cualitativas de investigación social. Madrid: Síntesis Editorial. 
216 Espinar \& Ocampo - Ways of Masculinity in Online Dating

Walker, L. \& Eller, J. (2016). Raising Capital: Claims of Masculinity among Men on Match.com. Men and Masculinities, 19(1), 42-63. doi: https://doi.org/10.1177/1097184X15575157

Wang, Y.J. (2012). Internet dating sites as heterotopias of gender performance: A case study of Taiwanese heterosexual male daters. International Journal of Cultural Studies, 15(5), 485-500. doi: https://doi.org/10.1177/1367877911422855

Eva Espinar-Ruiz is Associate Professor of the Department of Sociology II at the University of Alicante, Spain.

Ismael Ocampo is graduated in Sociology at the University of Alicante, Spain.

Contact Address: Direct correspondence to Eva Espinar-Ruiz, Department of Sociology II. Edificio de Ciencias Sociales, $1^{\mathrm{a}}$ planta,

Edificio 34, 03690 San Vicente del Raspeig Alicante (Spain), email:

eva.espinar@ua.es 


\section{Hipatia Press}

Instructions for authors, subscriptions and further details:

http://mcs.hipatiapress.com

\section{Masculinidades y Emociones como Construcciones Socioculturales: Una Revisión Bibliométrica}

Juan Carlos Ramírez Rodríguez, María del Pilar Gómez González' Norma Celina Gutiérrez de la Torre, Marcela Viridiana Sucilla Rodríguez ${ }^{1}$

1) Universidad de Guadalajara, Mexico

Date of publication: October $21^{\text {st }}, 2017$

Edition period: October 2017 - June 2018

To cite this article: Ramírez Rodriguez, J,C.; Gómez González, M.P;

Guitiérrez de la Torre, N.C \& Sucilla Rodriguez, M.V (2017). Masculinidades y emociones como construcciones socioculturales: una revisión bibliométrica. Masculinities and Social Change,6(3), 217-256

To link this article: http://doi.org/10.17583/MCS.2017.2734

\section{PLEASE SCROLL DOWN FOR ARTICLE}

The terms and conditions of use are related to the Open Journal System and to Creative Commons Attribution License (CC-BY). 


\section{Masculinities and Emotions as Sociocultural Constructions: A Bibliometric Review}

Juan Carlos Ramírez Rodríguez

Universidad de Guadalajara

Norma Celina Gutiérrez de la Torre Universidad de Guadalajara
María del Pilar Gómez González, Universidad de Guadalajara

Marcela Viridiana Sucilla Rodríguez1 Universidad de Guadalajara

\section{Abstract}

It was conducted a literature review on the relationship between masculinities and emotions, both understood as social constructs that respond to particular contexts. The principal aim is to describe the scientific production considering some parameters proposed by bibliometric studies. A comprehensive search of articles based on the definition of specific keywords and criteria according to a wide variety of databases of full text documents. The results of the systematization review show a very slow growth of these studies since the year 2000. More than a half of the studies were carried out in only two countries. There is a predominance of qualitative approach, particularly theoretical methods, such as ethnography, grounded theory and social constructionism. From the topics discussed in each of the studies, we propose a categorization to describe them and discuss implications of the emotions in the configuration of masculinities.

Keywords: Masculinities, emotions, state of the art 
MCS - Masculinities and Social Change Vol. 6 No. 3 October 2017 pp. 217-256

\section{Masculinidades y Emociones como Construcciones Socioculturales: Una Revisión Bibliométrica}

Juan Carlos Ramírez Rodríguez

Universidad de Guadalajara

Norma Celina Gutiérrez de la Torre Universidad de Guadalajara
María del Pilar Gómez González, Universidad de Guadalajara

Marcela Viridiana Sucilla Rodríguez1 Universidad de Guadalajara

\section{Resumen}

Se presenta el resultado de una revisión sistemática de la literatura sobre la relación entre masculinidades y emociones, entendidas ambas como construcciones sociales que responden a contextos particulares. El objetivo principal es mostrar una faceta de la producción científica considerando algunos parámetros propuestos por los estudios bibliométricos. El acopio de información se basó en la definición de palabras clave y criterios de búsqueda específicos de acuerdo con una amplia variedad de bancos de información de documentos de texto completo. Los resultados de la sistematización muestran un muy lento crecimiento de estos estudios a partir del año 2000. Más de la mitad de los estudios se han realizado en sólo dos países. Existe una predominancia de acercamientos cualitativos que parten de planteamientos teóricos diversos como la etnografía, la teoría fundamentada y el construccionismo social. A partir de los temas tratados en cada uno de los estudios se elaboró una categorización que muestra la amplitud e implicaciones que las emociones tienen en la configuración de las masculinidades.

Palabras clave: Masculinidades, emociones, estado del arte. 


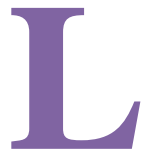

os estudios sobre género y emociones como construcciones sociales son un ámbito temático relativamente novedoso, en particular cuando nos referimos a la condición genérica de los hombres y las emociones. Si bien las emociones han sido aludidas en distintos momentos, contextos y por distintos autores (Bourdieu, 2000; Kaufman, 1997; Kimmel, 1997; Seidler, 2000, 2007), es hasta inicios de los años 80 que parece identificarse un incremento en las investigaciones sobre las mismas (Bericat Alastuey, 2014; Galasinski, 2004). Reconocemos la existencia de una amplia literatura sobre emociones que proviene principalmente de la psicología en la que se hace referencia a los hombres y en muchas ocasiones se compara con las mujeres (Galasinski, 2004; Shields, Garner, Di Leone, \& Hadley, 2007), no obstante, los acercamientos no plantean una discusión sobre los hombres en tanto sujetos genéricos. En este trabajo lo que nos interesa es identificar la literatura que parte de la tradición que establece una continuidad entre los feminismos, los estudios de género con mujeres y los estudios de masculinidad y que abordan las emociones como construcciones socioculturales, esto es, como resultado de procesos de socialización que corresponden a contextos históricos particulares y que son vividas individualmente (Enríquez Rosas, 2009; Gordon, 1990; Lutz \& White, 1986; Lutz, 1998). Forma parte de un proyecto de investigación más amplio a la vez que de una línea de investigación que hemos venido trabajando en los últimos años.

Tomando en consideración lo anterior, los cuestionamientos que guiaron el estudio que ahora presentamos son: ¿Cómo han crecido estos estudios? ¿Dónde se han realizado? ¿Qué temáticas se han investigado? ¿Qué orientaciones teóricas se han utilizado? Para responder estas preguntas consideraremos de utilidad algunos de los aportes hechos por los estudios bibliométricos que brindan una serie de criterios para documentar de manera sistemática la producción científica considerando indicadores de productividad, citación, contenido, metodología (Escorcia Otalora, 2008; González de Dios, Moya, \& Mateos Hernández, 1997) y procedencia (Romaní, Huaman, \& González Alcalde, 2011), entre otros (Agudelo, Bretón-López, \& Buela-Casal, 2003). Partiendo de lo anterior, el objetivo de este documento es mostrar una primera aproximación sobre las investigaciones que vinculan la masculinidad y las emociones entendidas 
como construcciones sociales. En una próxima publicación se discutirá en profundidad los debates que se han estado originando en estos estudios.

\section{Metodología}

Se realizó un análisis bibliométrico de los trabajos publicados entre 1985 y junio de 2016, los cuales son el resultado de una revisión sistemática de la literatura. Las fuentes de información fueron las bases de datos de texto completo que se encontraban disponibles en la red de bibliotecas de la Universidad de Guadalajara durante el periodo de búsqueda establecido y que a continuación se enumeran: Academic One File Unique; Annual Reviews; Cambridge Collection; Ebsco; Emmerald; Global Issues in Context; Informe Académico; Ingenta Connect; Jstor; Lippincott Williams \& Wolkins; ProQuest; Science Direct; Scopus; Web of Science; Wiley; Dialnet; Scielo; Eric y CONRICYT. El periodo de tiempo consultado abarca toda la información disponible hasta junio de 2016, incluyendo artículos empíricos y tesis doctorales, sin límite por país de origen, publicadas en español, inglés, portugués, francés, italiano y alemán.

Definimos como categorías centrales de búsqueda los términos "masculinidad" y "emociones". Con el propósito de hacer una revisión comprehensiva utilizamos varios criterios para diversificar, ampliar y profundizar la búsqueda, como son la utilización de sinónimos y términos o frases como partes del discurso contenido en el cuerpo del texto, de acuerdo con la propuesta elaborada por Rumsey (2008) como se muestra en la Tabla 1. 
Tabla 1

Categorías de búsqueda de los términos emociones y masculinidad

\begin{tabular}{|c|c|c|c|c|}
\hline \multirow[b]{2}{*}{ Concepto } & \multicolumn{2}{|c|}{ Español } & \multicolumn{2}{|c|}{ Inglés } \\
\hline & Emociones & Masculinidad & Emotions & Masculinity \\
\hline \multirow{8}{*}{ Sinónimos } & Emoción & Masculinidad & Emotion & Masculitiny \\
\hline & Emociones & Masculinidades & Emotions & Masculinities \\
\hline & Emocional & & Emotional & \\
\hline & Sentimientos & & Feeling & \\
\hline & Pasión & & Sentiments & \\
\hline & Afecto & & Passion & \\
\hline & Afectividad & & Affection & \\
\hline & & & Affectivity & \\
\hline \multirow{4}{*}{$\begin{array}{l}\text { Partes del } \\
\text { discurso }\end{array}$} & $\begin{array}{l}\text { Construcción } \\
\text { social de las } \\
\text { emociones }\end{array}$ & $\begin{array}{l}\text { Masculinidad } \\
\text { dominante }\end{array}$ & $\begin{array}{l}\text { Social } \\
\text { construction of } \\
\text { emotions }\end{array}$ & $\begin{array}{l}\text { Dominant } \\
\text { masculinity }\end{array}$ \\
\hline & $\begin{array}{l}\text { Antropología de } \\
\text { las emociones } \\
\text { Sociología de las } \\
\text { emociones }\end{array}$ & $\begin{array}{l}\text { Masculinidad } \\
\text { tradicional } \\
\text { Masculinidad } \\
\text { hegemónica }\end{array}$ & $\begin{array}{l}\text { Anthropology } \\
\text { of emotions } \\
\text { Sociology of } \\
\text { emotions }\end{array}$ & $\begin{array}{l}\text { Traditional } \\
\text { masculinity } \\
\text { Hegemonic } \\
\text { masculinity }\end{array}$ \\
\hline & $\begin{array}{l}\text { Manejo de } \\
\text { emociones }\end{array}$ & $\begin{array}{l}\text { Nuevas } \\
\text { masculinidades }\end{array}$ & $\begin{array}{l}\text { Labor of } \\
\text { emotion }\end{array}$ & $\begin{array}{l}\text { New } \\
\text { masculinities }\end{array}$ \\
\hline & Afrontamiento & & $\begin{array}{l}\text { Work of } \\
\text { emotion }\end{array}$ & \\
\hline
\end{tabular}

Fuente. Elaboración propia.

Los criterios de búsqueda que maneja cada base de datos son diferentes. La falta de uniformidad generó cierta complejidad que se ponderó en el proceso de búsqueda. Por ejemplo, Jstor tiene como motor de búsqueda "Item Title", en cambio Emmerald tiene "Keyword", lo que no es igual pero podría considerarse como equivalente. Por tal motivo definimos para cada base de datos un sistema de búsqueda particular que permitiera mantener criterios claros en el proceso de búsqueda actual, que si bien no son necesariamente homogéneos si guardan similitud, de manera que 
permita mantenerlos como referencia para futuras actualizaciones (Ver Anexo 1).

Para la selección de los documentos se revisaron el título, que este incluyera las palabras clave o sinónimos de género y emociones, el resumen y texto completo para verificar el cumplimiento de los criterios de selección, aquellos que eran resultado de estudios empíricos que aportaran una discusión teórica de los hombres como sujetos genéricos (masculinidad), así como aquellos que tenían un abordaje sobre las emociones como construcciones sociales.

Todos los documentos localizados fueron catalogados y almacenados en un repositorio para su análisis. Se elaboraron dos bases de datos para su manejo, la primera fue en EndNote que permitió hacer una organización, clasificación y depuración bibliográfica y la otra en Excel, para la posterior descripción y análisis del contenido de cada documento.

Para el análisis de la información se realizó una clasificación considerando variables, de acuerdo a ciertos indicadores bibliométricos (Agudelo et al., 2003; Escorcia Otalora, 2008; González de Dios et al., 1997; Romaní et al., 2011) como: número de publicaciones, en el cual se incluyó número de publicaciones por año, número de tesis y de artículos, así como el promedio de publicaciones; autoría, entre las cuales está el número de publicaciones como autor único, coautor, número de coautores; idioma y país, número de publicaciones por idioma, país de publicación y adscripción institucional del autor/a principal; temática tratada, población estudiada, acercamientos teóricos utilizados, metodología planteada, técnicas de investigación utilizadas.

\section{Resultados}

A continuación presentamos una perspectiva general de los hallazgos de la revisión. Se recuperaron 156 documentos que incluyen artículos y tesis de doctorado, de los cuales 78 cumplieron con un componente doble, por una parte una argumentación teórica sobre masculinidad y emociones y por otra, investigación de campo con información empírica, lo cual permite la comprensión del fenómeno social de interés en poblaciones específicas (Bernard, 1995). 
El inicio de las publicaciones fue el año 1985, que se definio a posteriori, es decir, una vez recolectada la información, se identificó el articulo más antigüo y con respecto al año de finalización, este fue establecido por los investigadores previo al proceso de busqueda.

De los 78 trabajos incluidos y que se han publicado en los últimos 30 años, $85 \%$ corresponden a artículos y el porcentaje restante a tesis doctorales. El promedio de publicación fue de 2.4 documentos anuales. Sin embargo, como se muetra en la gráfica 1, su distribución no es uniforme a lo largo del tiempo. Del primer documento registrado siguieron cuatro años sin un solo texto. Durante las decadas de 1980 y 1990 apenas se registra un acumulado de $6 \%$ y es a partir de entrado el nuevo milenio en que se empieza a observar una tendencia creciente alcando el mayor número de documentos en el año de 2012 para enseguida decrecer. En este sentido, se podría decir que la intersección de temas aquí explorados apenas ha empezado a atraer la atención de la comunidad científica, siendo una ventana de oportunidad para la investigación y que requiere de esfuerzos sostenidos para describir y analizar dicha intersección.

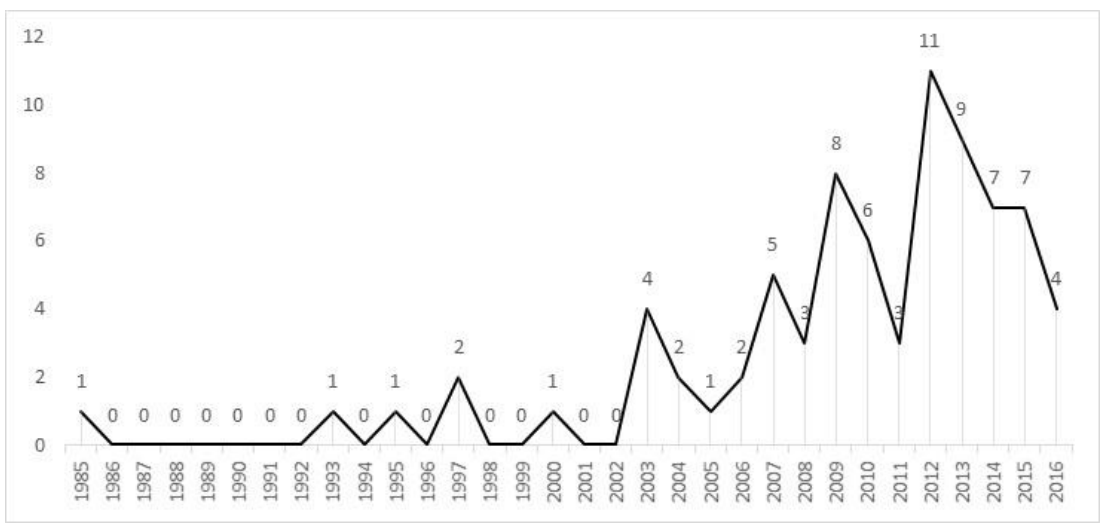

Gráfica 1. Número de documentos publicados sobre masculinidad y emociones según año de aparición 
Existe un predominio del inglés como idioma de publicación de los documentos y en menor proporción en español y portugués (ver Tabla 2), no es de extrañar por varias razones, en primer término porque el lugar en que se han desarrollado estos estudios es en países anglohablantes (ver Tabla 3) con predominio de Estados Unidos y Reino Unido, en segundo lugar porque no obstante hay investigaciones desarrolladas en sociedades hispanohablantes, la publicación se hizo en revistas que su idioma de publicación es el inglés. Por otra parte, existe un $12 \%$ de estudios en español, provenientes de países como: México, Colombia, España, Perú y Puerto Rico y el porcentaje restante representa los estudios publicados en portugués.

Tabla2

Idiomas en que han sido publicados los documentos

\begin{tabular}{lcc}
\hline Idioma & Núm & $\%$ \\
\hline Ingles & 66 & 85 \\
Español & 9 & 12 \\
Portugués & 3 & 4 \\
\hline Total & 78 & 100 \\
\hline
\end{tabular}

Fuente. Elaboración propia.

Tabla 3

País donde se realizó el estudio

\begin{tabular}{lcc}
\hline $\begin{array}{l}\text { País donde se realizó el } \\
\text { estudio }\end{array}$ & Núm & $\%$ \\
\hline Estados Unidos & 28 & 36 \\
Reino Unido & 12 & 15 \\
Australia & 5 & 6 \\
México & 4 & 5 \\
\hline
\end{tabular}


Tabla 3

Continuación

\begin{tabular}{lcc}
\hline País donde se realizó el & Núm & $\%$ \\
\hline estudio & 4 & 5 \\
Canadá & 3 & 4 \\
Brasil & 3 & 4 \\
Inglaterra & 3 & 4 \\
España & 2 & 3 \\
Colombia & 2 & 3 \\
Suecia y Noruega & 2 & 3 \\
Chile & 2 & 3 \\
Suráfrica & 1 & 1 \\
Ghana & 1 & 1 \\
Irlanda & 1 & 1 \\
Israel y Palestina & 1 & 1 \\
Perú & 1 & 1 \\
Noruega & 1 & 1 \\
Puerto Rico & 2 & 3 \\
Sin dato & 78 & 100 \\
\hline Total & &
\end{tabular}

Fuente. Elaboración propia

\begin{abstract}
Al revisar las instituciones donde se realizan los estudios, no se pudo identificar alguna que se considere como referente en la temática de interés, más bien existe una gran cantidad de instituciones, sobre todo, de educación superior de distintos lugares que están empezando a estudiar estos temas. Asimismo, el número de autores con más de una publicación sobre el tema es limitado, siendo 5 autores con un máximo de 2, ver Tabla 4.
\end{abstract}


226 Ramírez Rodríguez et al. - Masculinidades y Emociones

Tabla 4

Número de publicaciones por autor

\begin{tabular}{lcc}
\hline Publicación por autor & Núm & $\%$ \\
\hline Única publicación & 68 & 93.1 \\
Dos publicaciones & 5 & 6.9 \\
Total & 73 & 100 \\
\hline
\end{tabular}

Fuente. Elaboración propia

$50 \%$ de las publicaciones corresponden al trabajo de autor único y los demás son en coautoría, de las cuales aquellos de 2 y 4 coautores son los frecuentes, ver Tabla 5 y 6.

Tabla 5

Número de publicaciones según cantidad de autores

\begin{tabular}{lcc}
\hline Autores por publicación & Núm & $\%$ \\
\hline Autor único & 40 & 51 \\
Coautorías & 38 & 49 \\
\hline Total & 78 & 100 \\
\hline
\end{tabular}

Fuente. Elaboración propia

Tabla 6

Cantidad de autores en los documentos con Coautoría

\begin{tabular}{|c|c|c|}
\hline Cantidad de autores & Núm & $\%$ \\
\hline 2 & 17 & 45 \\
\hline 3 & 8 & 21 \\
\hline 4 & 10 & 26 \\
\hline 5 & 2 & 5 \\
\hline 6 & 1 & 3 \\
\hline Total & 38 & 100 \\
\hline
\end{tabular}

Fuente. Elaboración propia 
Por otra parte, las revistas que están publicando estos trabajos son diversas, por ejemplo, la revista que más publicaciones sobre el tema es el "Journal of Aging Studies" con tres. Trece revistas tienen un total de 2 artículos publicados y 52 revistas tienen un artículo publicado cada una. Por otra parte se identificaron 10 tesis doctorales, distribuidas en 2 de sociología, 3 de psicología y 7 de filosofía.

Como se puede ver en la Tabla 7, el marco teórico más utilizado en los estudios corresponden a etnografía (14\%), el construccionismo social y teoría fundamentada cada uno con una proporción de $13 \%$, cabe destacar que en muchos casos, más del $20 \%$, no se especificó el marco teórico con el cual se abordó el fenómeno de estudio. El resto reporta una diversidad de acercamientos como las teorías feministas y de género, representaciones sociales, sociología de las emociones y el interaccionismo simbólico, entre otros.

Tabla 7

Aproximación teórica

\begin{tabular}{lcc}
\hline Aproximación Teórica & Núm & $\%$ \\
\hline Etnografía & 11 & 14 \\
Construccionismo social & 10 & 13 \\
Teoría fundamentada & 10 & 13 \\
Teoría de Género & 4 & 5 \\
Constructivismo social & 3 & 4 \\
Clasificación de Hochschild & 2 & 3 \\
Representaciones sociales & 2 & 3 \\
Interaccionismo simbólico & 1 & 1 \\
Sociología de las emociones & 1 & 1 \\
Otras & 16 & 21 \\
Sin dato & 18 & 23 \\
\hline Total & 78 & 100 \\
\hline
\end{tabular}

Fuente. Elaboración propia 
La mayor parte de los estudios realizados han utilizado un enfoque metodológico cualitativo, correspondiente a $82,1 \%$, la proporción de estudios cuantitativos es de $10,3 \%$, lo cual representa que por cada estudio con enfoque cuantitativo se hacen 10,6 estudios cualitativos. La metodología mixta tiene menor frecuencia entre los documentos revisados (Tabla 8).

Teniendo en cuenta las temáticas de interés, emociones y masculinidades, el enfoque metodológico que puede dar una mejor compresión del fenómeno es el cualitativo, debido a que este permite reconocer los procesos subjetivos, los significados, creencias, valores, percepciones y normativas que subyacen como elementos explicativos del fenómeno social de interés (Creswell, 2012).

Tabla 8

Enfoque metodológico utilizado

\begin{tabular}{lcc}
\hline Enfoque & Núm & $\%$ \\
\hline Cualitativa & 64 & 82.1 \\
Cuantitativa & 8 & 10.3 \\
Mixta & 6 & 7.7 \\
\hline Total & 78 & 100 \\
\hline
\end{tabular}

Fuente. Elaboración propia

Como se puede observar en la Tabla 9, la técnica de investigación más utilizada en estos estudios fue la entrevista, ya sea identificada como sólo entrevista o entrevista en profundidad, entrevista psicosocial; otra opción utilizada fue la combinación de técnicas, la entrevista y diario de campo, observación participante, grupo de discusión o focal, video, encuesta. El grupo focal o de discusión fue otro recurso, aunque menos frecuente; en algunas ocasiones de conjuntó con otra técnica como diario de campo y fotografía. En un caso se utilizó el grupo de discusión en línea, esto es, la opción no presencial es un recurso poco visto y que convendría analizar en detalle las implicaciones que tiene. El recurso del video es interesante en tanto que las emociones generalmente son recuperadas a través del discurso y en ocasiones recurriendo a descripciones sobre la afectación emocional con que se hace el relato, al menos en las entrevistas, esto es la información 
que se recupera por la observación, pero esto no es una constante. El video abre posibilidades de análisis múltiples, pero como se deja ver, no es una opción socorrida sino más bien una excepción.

Tabla 9

Técnicas utilizadas en los estudios

\begin{tabular}{lcc}
\hline Técnicas utilizadas & Núm & $\%$ \\
\hline Entrevistas & 30 & 38 \\
Entrevistas y otra técnica & 19 & 24 \\
Otro & 9 & 12 \\
Grupo focal/Discusión grupal & 7 & 9 \\
Sin dato & 7 & 9 \\
Encuesta & 6 & 8 \\
\hline Total & 78 & 100 \\
\hline
\end{tabular}

Fuente. Elaboración propia

En el subcampo de los estudios de género de los hombres, las temáticas tratadas en los últimos 30 años se han multiplicado y profundizado, como se ha mostrado en el mundo, en América Latina y en México (Gutmann, 2004; Kimmel, Hearn \& Connell, 2005; Ramírez Rodríguez \& Cervantes Ríos, 2013; Ruspini, Hearn, Pease et al., 2011). Temáticas relacionadas con la familia como es la paternidad ha sido una preocupación central, al igual que el desciframiento de la sociedad heteronormativa y heterosexual como eje configurador de la masculinidad dominante; el trabajo como eje estructural que da sentido a la identidad y favorece o dificulta el cumplimiento de mandatos centrales de género, como es el del hombre proveedor anclado a fases específicas del ciclo de vida que marcan el tránsito hacia la adultez, prueba de la madurez como sujeto masculino que muestra integridad. También la violencia o las violencias como formas estructurantes de prácticas e (inter)subjetividades de género de los hombres, algunas de ellas cada vez más cuestionadas como la violencia en las relaciones de pareja, en los espacios laborales y públicos que adquieren modalidades de acoso sexual en el trabajo y en la vía pública. Pero otras violencias en que el género apenas empieza a asomarse como elemento 
explicativo y que es causal de extensas y variadas formas de ejercicio de violencias como las ligadas al crimen organizado, la trata de personas, vinculadas con formas de socialización en que priman relaciones homosociales, en las cuales los pactos patriarcales se reafirman cotidianamente. No es de extrañar que en tales ámbitos temáticos haya empezado a llamar la atención el vínculo con las emociones en tanto dispositivos aprendidos socialmente, que regulan las relaciones y reafirman o cuestionan las asimetrías que se mantienen entre hombres y mujeres y entre hombres.

En la Tabla 10, mostramos la clasificación que hemos elaborado de los documentos revisados, se realizó en varias etapas, lo primero fue identificar el tema central del documento y posteriormente estas temáticas fueron agrupadas en catorce categorías, éstas son: ciclo de vida; cuerpo; raza; cultura; cultura emocional; trabajo emocional; espacio público y relaciones sociales; familia; relaciones interpersonales; salud; sexualidad; trabajo; violencias, vulnerabilidad y estructura social. Esta clasificación permitió de manera simplificada dar cuenta de las tendencias en cuanto a los fenómenos que han resultado de mayor interés en términos de investigación en el periodo contemplado.

Tabla 10

Ámbitos temáticos tratados sobre emociones y masculinidad

\begin{tabular}{ll}
\hline Categorías & Temas \\
\hline $\begin{array}{l}\text { Ciclo de } \\
\text { vida }\end{array}$ & Juventud, Adolescentes, Intergeneracionalidad, Vejez \\
Cuerpo & Cuerpo, Estética \\
Raza & Raza \\
& Nacionalidad, Identidad de género, Identidad sexual, Estereotipos, \\
Cultura & $\begin{array}{l}\text { Identidad, Humor, Justicia, Estigma, Memoria, Música, } \\
\text { Representaciones sociales, Roles de género }\end{array}$
\end{tabular}


Tabla 10

Continuación

\begin{tabular}{|c|c|}
\hline $\begin{array}{l}\text { Cultura } \\
\text { emocional }\end{array}$ & $\begin{array}{l}\text { Emociones específicas, Trabajo emocional, Regulación } \\
\text { emocional, Expresión emocional, Apoyo emocional, Bienestar, } \\
\text { Duelo, Expresión afectiva, Manejo emocional, Reacción } \\
\text { emocional, Significado }\end{array}$ \\
\hline $\begin{array}{l}\text { Espacio público y } \\
\text { relaciones } \\
\text { sociales }\end{array}$ & $\begin{array}{l}\text { Deportes, Pares, Ámbito escolar, Ámbito universitario, Espacio } \\
\text { público, Medios de comunicación, Socialización, Conflicto, } \\
\text { Homosocialización }\end{array}$ \\
\hline Familia & $\begin{array}{l}\text { Paternidad, Familia, Abuelidad, Jefatura familiar, Separación, } \\
\text { Viudez }\end{array}$ \\
\hline $\begin{array}{l}\text { Relaciones } \\
\text { interpersonales }\end{array}$ & $\begin{array}{l}\text { Intimidad, Pareja, Amistad, Relaciones de poder, Pares, } \\
\text { Relaciones interpersonales }\end{array}$ \\
\hline Salud & Salud, Salud mental, Infertilidad, Duelo, Suicidio, Alcoholismo \\
\hline Sexualidad & Sexualidad, Identidad sexual, Cortejo, Prostitución \\
\hline Trabajo & $\begin{array}{l}\text { Ocupación, Desempleo, Migración, Conciliación familia- } \\
\text { trabajo, Economía, Trabajo }\end{array}$ \\
\hline Violencia & Violencia, Armas, Seguridad \\
\hline Vulnerabilidad & Cuidados, Autocuidado, Marginación, Vulnerabilidad \\
\hline Estructura social & Neoliberalismo, Justicia, Nacionalidad, Movimientos sociales \\
\hline
\end{tabular}

Fuente. Elaboración propia

Resulta pertinente mencionar que los fenómenos abordados en las publicaciones pueden clasificarse en diferentes categorías, porque algunos de los documentos abordaron dos o más temáticas como se muestra a continuación.

En la primera categoría, ciclo de vida, incorporamos las temáticas: juventud, adolescentes, intergeneracionalidad y vejez; los relacionados con la juventud son los más numerosos (4) mientras que los otros temas cuentan con un solo texto para cada una de ellas. Ciclo de vida se relaciona con la categoría Familia en que integramos paternidad, familia propiamente dicha, abuelidad, jefatura familiar, separación y viudez con un total de 15 
documentos; siendo los dos primeros temas mencionados los que cuentan con la mayoría de las publicaciones encontradas, 6 para el caso de la paternidad y 5 para familia, los otros cuatro grupos cuentan con una sola publicación en cada una de ellas. Íntimamente vinculado con aspectos de familia está el ciclo de vida. Las relaciones de cortejo, previas al establecimiento de relaciones de pareja, pero también problemáticas particulares de hombres jóvenes, adolescentes o niños transitan por procesos complejos en que se van construyendo las distintas configuraciones de masculinidad. En ellas las emociones juegan un papel central, porque los espacios de relaciones de pares son ámbitos homosociales que legitiman formas particulares de concebirse como sujetos de género, se ponen en tensión los aprendizajes de socialización primaria y secundaria, esto es, la formación recibida en el seno familiar, que se contrapone, en no pocas ocasiones, a lo demandado por el grupo de amigos, compañeros en la escuela, en el espacio recreativo; en tales relaciones es que los pares actúan como reguladores emocionales, en que el miedo a la crítica, al rechazo, parecen juegar un papel clave, al igual que la aceptación del grupo que va fortaleciendo y moldeando la identidad. Por su parte hombres de la tercera edad y jubilados, tienen una proclividad a alejarse de una configuración masculina caracterizada por el distanciamiento emocional, la rudeza física y arriesgada, tornándose más cercanos con los nietos a quienes dispensan muestras de amor e intimidad, de manera que hay relaciones intergeneracionales nutricias entre hombres, siendo las emociones dispositivos que las posibilitan.

En la categoría Cuerpo se encontraron 5 documentos los cuales se relacionan básicamente con la práctica deportiva como el tae kwon do, las luchas y el surf; el contacto físico y el ejercicio corporal, en particular, en la práctica del surf emergen emociones como alegría, miedo, ansiedad, culpa, orgullo, disgusto, vergüenza, las cuales se vinculan directamente con el tema de las emociones específicas, ubicada dentro de la categoría de Cultura emocional; también se encontró un documento relacionado con la estética corporal en hombres jóvenes que fue ubicado dentro de la misma categoría.

Llama la atención el número reducido de estudios sobre deporte, emociones y masculinidades, en particular las relacionadas con el fútbol soccer que ha sido investigado como un espacio en el que las emociones en 
los hombres afloran con libertad y han sido señaladas por ser altamente violentos e inseguros en algunos contextos (Javaloy Mazón, 1996; Martín Cabello, 2011).

La raza es la tercera categoría establecida en la clasificación, esta es considerada como un elemento importante dentro de la construcción de la masculinidad, que incluye ideales sociales y culturales como es la masculinidad hegemónica, a través de la cual se plantean características y normas, que al no ser cumplidas, deja en desventaja a aquellos que no lo hacen con respecto a las jerarquía y relaciones de poder dentro de una sociedad (Kimmel, 1997), como por ejemplo la población negra, que en diversos contextos, ha sido etiquetada y estigmatizada, en contraste a esto, en los documentos revisados, se aborda como los individuos pertenecientes a estos grupos generan resistencia y lucha para enfrentar estereotipos como la violencia, el peligro, la inseguridad y la pobreza, a través de la amistad, la unión entre pares, la camaradería, así como el control al interior de grupos para el manejo de emociones negativas que puedan reproducir y legitimar estos estereotipos en los grupos sociales en los que se encuentran. Esta categoría incluye 5 documentos, los cuales se vinculan con la categoría del espacio público, específicamente en el ámbito universitario y laboral y con la categoría de la cultura emocional en la que se agruparon tópicos relacionados con emociones específicas como: orgullo, angustia, ansiedad, tristeza, vergüenza, culpa, frustración, melancolía, tristeza y depresión.

En la categoría de cultura se incorporaron un total de 18 publicaciones distribuidas en 12 temáticas a saber: nacionalidad, identidad de género, identidad sexual, estereotipos, identidad, humor, justicia, estigma, memoria, música, representaciones sociales y roles de género; resulta pertinente señalar que la mayoría de estas temáticas cuentan con un solo documento publicado, siendo las temáticas de identidad de género (3), identidad sexual (3), estereotipos (2) e identidad (2) las que más publicaciones tienen. Al igual que lo que sucede con otras categorías, esta se entrecruza con diversas temáticas ubicadas en otras categorías especialmente con las relacionadas con la cultura emocional y el trabajo emocional.

Cultura emocional es la categoría que representa el mayor conjunto de documentos (62), esto es debido a que los temas en ella contenidas se vinculan prácticamente con las demás categorías y temáticas establecidas. Dicha categoría hace referencia a lo que se asocia con distintas facetas de 
las emociones, desde las etiquetas, valores, normas de expresión y regulación, formas, espacios y situaciones para la expresión de ciertas emociones, que según el contexto, el momento y la cultura son permitidas socialmente (Hochschild, 2003, 2007; Lutz, 1998). Las temáticas que se incluyeron fueron emociones específicas, trabajo emocional, regulación emocional, expresión emocional, apoyo emocional, bienestar, duelo, expresión afectiva, manejo emocional, reacción emocional y significado; sin embargo, es necesario enfatizar que emociones específicas, trabajo emocional y regulación emocional, son las más abordadas con 27, 13 y 9 documentos respectivamente, mientras que el resto de los temas cuentan con un número considerablemente más bajo de publicaciones.

La categoría de espacio público y relaciones sociales, podría considerarse limitada en cuanto a número de publicaciones, 17 en total, si referimos que son precisamente estos ámbitos los considerados tradicionalmente como "entornos masculinos", en esta categoría hemos ubicado: deportes, pares, ámbito escolar, ámbito universitario, espacio público, medios de comunicación, socialización, conflicto y homosocialización; siendo el grupo de deportes el que más publicaciones tiene con un total de 4 documentos.

La categoría relaciones interpersonales si bien puede ser considerada transversal dado que en todos los fenómenos se encuentra presente el aspecto relacional entre seres humanos y es a partir de esa interacción que se generan las construcciones de significados y prácticas (Gordon, 1990), por ejemplo en el espacio militar, donde se crean lazos de amistad, pero con férreo control de emociones como el miedo, porque ante todo está la demostración de una configuración de la masculinidad específica, que entre otros consiste en el rechazo de comportamientos que se puedan asociar con vulnerabilidad considerada como femenizante (Kimmel, 1997), ello provoca consecuencias como problemas de salud, debido a la resistencia a evidenciar necesidades, dolencias que puedan ser entendidas como una amenaza para la identidad masculina (Sabo, 2005). Existen otros comportamientos o construcciones que difieren de lo que en algunos artículos se reconoce como masculinidad hegemónica, como lo es el tener expresiones afectivas entre hombres y que no son sinónimo de homosexualidad. También en esta categoría se incluyen la intimidad, las relaciones de pareja, los vínculos de amistad, las relaciones de poder, los 
vínculos entre pares y las relaciones interpersonales mismas, siendo estas últimas las aglutinadas en esta categoría con un total de 13 documentos, que van de 1 a 3 publicaciones por temática.

En la salud ubicamos temas como de salud física como de, salud mental, infertilidad, duelo, suicidio y alcoholismo con un total de 15 documentos; es en las dos primeras temáticas que se encuentran la mayoría de los artículos clasificados, con 5 publicaciones en cada uno de los casos; llama la atención que el fenómeno del alcoholismo sea poco abordado debido a que solamente cuenta con una publicación, si consideramos que el consumo de bebidas alcohólicas genera trastornos graves de salud, está asociada a un nivel elevado de accidentes automovilísticos y a las violencias entre pares y contra la pareja (Brandes, 2004; Menéndez, 1990; Sabo, 2005; Treviño Siller, Villanueva Borbolla, Marcelino Sandoval, \& Álvarez Guillén, 2014). Además el consumo de bebidas alcohólicas entre hombres es una práctica homosocial que reafirma configuraciones específicas de masculinidad en diversos contextos sociales (Gutmann, 2000).

Sobre sexualidad encontramos un total de nueve documentos, siendo la temática de la sexualidad misma la que cuenta con un mayor número de artículos (cuatro), se ubicaron tres publicaciones sobre identidad sexual, un documento sobre el proceso del cortejo en hombres jóvenes y otro, sobre el ejercicio de la prostitución; en los artículos mencionados se abordan aspectos relacionados con el inicio de la vida sexual activa en hombres jóvenes, la disfunción eréctil, la funcionalidad sexual y los servicios médicos, la infidelidad masculina, relacionados con emociones particulares como el amor, el deseo, la tristeza, la ternura, el miedo, la impotencia, la ira y los celos; la reducida cantidad de documentos identificados dan cuenta de una aparente escasez de investigaciones científicas en las que se vincule la sexualidad con las masculinidades y las emociones, lo que resulta un tanto paradójico, dado a que es precisamente el ámbito de la sexualidad uno de los espacios relacionales donde se expresan mayormente las emociones.

Por otra parte, resulta pertinente señalar que son las investigaciones relacionadas con la sexualidad y el ejercicio de prácticas sexuales riesgosas de los hombres en diferentes contextos, las que presentan evidencias científicas de la presencia de serias problemáticas de salud pública tales como: las enfermedades de transmisión sexual (ETS), el VIH-sida, el embarazo adolescente no intencionado, el aborto, etc. que 
tienen también repercusiones sociales desfavorables como el rechazo, la discriminación, la homofobia y las violencias, que pueden originar enojo, impotencia, frustración, ira, entre otras emociones vinculadas con rígidos estereotipos y roles de género.

La categoría trabajo es una de las que cuenta con un número importante de publicaciones, al ser un total de 16 trabajos, de los cuales la temática particular de la ocupación es en la que más investigaciones se han realizado, con un total de nueve, mientras que el desempleo y la migración tienen solamente dos documentos, en el caso de la migración es pertinente señalar que, si bien la mayoría de los hombres migran por cuestiones que tienen que ver con la falta de oportunidades laborales (Hernández Sánchez, 2008) también puede ser por otras cuestiones vinculadas con las violencias (inseguridad, guerras, etc.) (Asakura \& Torres Falcón, 2012), por una orientación sexual diferente, que busca ser ocultada a la familia (Kobelinsky, 2012); los otros temas que incorporamos en esta categoría son los de conciliación, economía y trabajo mismo, éstas últimas cuentan con una publicación de cada una de ellas, esta categoría aborda elementos muy sensibles para los hombres por lo que en las publicaciones registradas no es de extrañar que dichas temáticas se encuentren vinculadas con sentimientos de orgullo por el desempeño, el status, la valoración social que se deriva de logros en el trabajo, por tanto, del cumplimiento del mandato de proveedor. Por otro lado, angustia, ansiedad, tristeza, vergüenza, sentimiento de culpa, cuando no se es posible satisfacer las necesidades demandadas por la familia, por la descendencia. Emociones que surgen de la interacción social con la pareja y los hijos e hijas a causa de la pérdida del empleo. Además las modificaciones de las expectativas laborales que se transforman conforme los períodos de desempleo se prolongan y los apoyos familiares se van transformando en recriminaciones por el deterioro en el nivel de vida. La identidad de género, la masculinidad se erosiona; pero también se observan salidas alternas, en que se reajustan los papeles de proveduría, se flexibilizan las posiciones a veces de manera provisional o se adoptan como un nuevo arreglo definitivo entre algunos hombres y mujeres, cambiándose o combinándose la provisión de cuidados a la prole y las tareas domésticas. Ante condiciones de cambio económico estructural la resignificación del trabajo asociado al género demanda también la profundización del conocimiento de las emociones que están entretejidas a estos procesos, 
emociones que son diversas, en ocasiones coincidentes, pero también contrapuestas.

Las violencias en sus diferentes manifestaciones se presentan en "escenarios naturalizados" en los que se concibe, ejerce y reafirma una manera de construirse como sujetos de masculinidad (Ramírez Rodríguez, 2010). En esta categoría identificamos diez artículos en los que aparecen formas de expresión o ejercicio de las violencias disímbolas, desde las asociadas a movimientos sociales hasta las interpersonales. Las emociones o mejor dicho, ciertas emociones han sido concebidas como objeto de represión, de control, porque no son compatibles con configuraciones de género de los hombres, fincadas en el control, la fuerza, la competencia que hacen una continuidad con la ira, el enojo, el miedo y que se articulan a prácticas y conductas como la agresión y la coerción. La supresión de las emociones indispensables para la dominación como la compasión, el dolor, entre otras, no parecen formar parte de este espectro de emociones. Se detectan escenarios diversos de asociación entre violencia y emociones entre ellos la violencia contra la pareja en la que puede presentarse una desconexión emocional y el episodio de violencia o bien, la percepción, valoración de la gestualidad de la mujer significada como miedo, como sorpresa por parte del hombre para entonces manipular las emociones como el enojo que éste último actualiza a manera de un performance. Así se ponen en juego elementos cognitivo conductuales. Atender las emociones como construcciones sociales evidencia su maleabilidad y puede poner en cuestionamiento la asociación "natural" entre enojo - agresión - violencia masculinidad. La participación de hombres jóvenes en movimientos sociales muestra una resignificación del enojo ante las injusticias sociales, ahí el enojo se vincula con activismo social, luchas reivinicativas sin violencia y sin agresión, incluso ante situaciones de provocación. El contexto adquiere una vital relevancia en que la emocionalidad es un dispositivo para la acción.

La vulnerabilidad es una condición importante en el proceso de construcción de la identidad genérica de una proporción de hombres, lo cual se ha establecido como una característica evitable debido a que va en contra de lo socialmente aceptado en un hombre, es decir, la fuerza, la rudeza, la tolerancia al dolor, ya sea físico y/o emocional, en esta categoría se incorporaron temáticas o condiciones que colocan a los hombres en 
posiciones que pueden cuestionar su identidad genérica al tener que depender de terceras personas: pareja, hijas/os, nietas/nietos, nueras, vecinas, o personal profesional contratado para el cuidado que deben dispensarles cuando atraviesan situaciones en las que han perdido la capacidad de autonomía, lo que se relaciona con emociones específicas tales como la depresión, la tristeza, la vergüenza y con estrategias de regulación emocional para enfrentar tales circunstancias (temáticas encontradas dentro de la categoría de la cultura emocional); las temáticas agrupadas en esta categoría son: cuidados, autocuidado, marginación y vulnerabilidad; resulta pertinente señalar que el tema del cuidado tanto de quien lo proporciona como de quien lo recibe había sido tradicionalmente concebido como una tarea de las mujeres, la participación de los hombres como cuidadores abre la discusión sobre el replanteamiento y cuestionamiento de la división sexual del trabajo.

Finalmente, en la categoría de estructura social, incorporamos tópicos en el nivel macro tales como el neoliberalismo, la justicia, la nacionalidad y los movimientos sociales, en los que se encontró solamente un artículo por cada una de las temáticas establecidas, el hecho de que éstos se inserten en marcos estructurales más amplios puede ser parte de la razón por la que existen tan pocos documentos dado que las emociones y las masculinidades han sido mayormente abordadas desde enfoques cualitativos en niveles meso y microsociales.

\section{Un Cierre Preliminar}

A partir de la revisión inicial de la literatura, podemos señalar que el estudio de la intersección entre emociones y masculinidades, entendidas como construcciones socioculturales, se encuentra en una etapa inicial de desarrollo. La tendencia es al incremento del número de publicaciones, pero en los últimos años presenta una ligera disminución. La literatura publicada está escrita fundamentalmente en inglés y se manera muy secundaria en español y portugués, siendo los países anglosajones en donde más se han desarrollado estas investigaciones. Existe un predominio en el uso de acercamientos teórico-metodológicos cualitativos, en particular la teoría fundamentada, la etnografía y el constructivismo social. Cabe señalar que en una proporción importante de los reportes de investigación hay una 
ausencia en el señalamiento de la discusión teórica que fundamenta los estudios. El aparato instrumental predominante es acorde con el enfoque cualitativo, como entrevistas, trabajo en grupos focales o de discusión entre otros. Pero también se identificaron estudios cuantitativos y mixtos en menor proporción. Las temáticas que se estudian son muy diversas y no se puede identificar una tendencia o predominancia. Se podría decir que se encuentra en una etapa exploratoria de diversos ámbitos temáticos.

\section{Fuentes Consultadas}

Es necesario aclarar que la literatura que se ha recuperado y sistematizado proviene de las bases de datos de revistas de texto completo disponibles en repositorios académicos, tanto de la Universidad de Guadalajara como de otras Instituciones de Educación Superior que forman parte del CONRICYT. En tal sentido, hasta ahora se ha excluido la literatura que trata el tema y que se encuentra publicada en formatos distintos como libros, revistas y reportes que no forman parte de los acervos consultados. Hemos identificado una serie de artículos que al parecer cumplen con los criterios que definimos y describimos más arriba, pero que no pudimos acceder a su versión de texto completo y por tanto no se incluyeron.

Tenemos particular interés por los estudios sobre género y emociones en la región de América Latina, en especial aquellos que se enfocan en hombres y las masculinidades. Una parte de estos estudios están publicados en formatos como tesis de postgrado o artículos en revistas no indexadas o que si lo están, forman parte de repositorios institucionales independientes, cuya búsqueda y acceso es más laborioso y requiere mayor inversión de tiempo. Este proceso lo estamos llevando a cabo a fin de complementar lo que hemos expuesto.

\section{Las Categorías de Agrupación}

Aquí se ha hecho una categorización y tematización de los trabajos publicados. Este ha sido un proceso de clasificación a posteriori. Como toda clasificación responde a los intereses de quien la elabora. Asumiendo este sesgo, consideramos que la misma ofrece una perspectiva general de la diversidad y también de la especificidad de los estudios que se han venido 
desarrollando. Las diferentes categorías y temas se imbrican lo que enriquece los propios estudios, los complejiza y muestra la importancia que tienen las emociones como objeto de análisis que aporta otra ventana de comprensión de las prácticas y subjetividades que configuran las masculinidades.

\section{Perspectiva Analítica de Futuro}

El acercamiento descriptivo expuesto de los estudios sobre la relación entre emociones, hombres y masculinidades es la primera etapa del trabajo que estamos desarrollando para construir el estado del arte. Se encuentra en proceso un segunda etapa que consiste en mostrar los debates teóricos y metodológicos de la literatura consultada, esto es, como se está concibiendo y utilizando la teorización sobre el género de los hombres (Bourdieu, 2000; Connell, 2003; Holter, 2005) y sobre las emociones (Gordon, 1990; Le Breton, 2009; Turner \& Stets, 2009); de manera particular nos interesa identificar diferentes facetas, por ejemplo: si el estudio de las emociones contribuyen o no en la (re)producción de (a)simetrías sociales de género, en qué sentido y con qué profundidad; independiente de las temáticas que se estudian ¿se identifican constelaciones de emociones que tienen un significado relevante para los hombres y la identidad genérica?, si es así ¿cuáles y qué implicaciones tienen? Las emociones son objeto de regulación basadas en creencias, valores, contextos y significados, parecería que existe un trabajo emocional constante por parte de los hombres, lo que demanda atención, recursos y energía, ¿cuáles son los recursos pedagógicos que entran en juego para conformar la emocionalidad expresada por los hombres en función del ciclo de vida y los grupos de pares del que forma parte? Algunos autores, entre ellos Seidler (2000; 2007) y Kaufman (1997) han considerado que la apertura a la expresión emocional por parte de los hombres es una vía que modifica la configuración de la masculinidad y favorece el establecimiento de relaciones más igualitarias ¿Este argumento puede sostenerse con la información disponible o bajo qué circunstancias esto ocurre? Estos son algunos de los cuestionamientos que nos interesa explorar. Pensamos que el estudio de las emociones como construcciones sociales está abriendo un camino de comprensión que se revelará fundamental no sólo para la comprensión de los procesos de construcción 
sociogenérica de nuestras sociedades y en particular de los hombres y las masculinidades, sino para contribuir en la elaboración de propuestas de transformación tendientes a una sociedad más igualitaria y equitativa entre hombres y mujeres y entre hombres.

\section{Bibliografía}

Abelson, M. J. (2014). Men in context: Transmasculinities and transgender experiences in three US regions. (3644415 Ph.D.), University of Oregon, Ann Arbor. Retrieved from http://wdg.biblio.udg.mx:2048/docview/1625050131?accountid=28 915.

Adams, L. S. (1997). Sex differences in reports of emotional experiences. (9725022 Ph.D.), Emory University, Ann Arbor. Retrieved from http://wdg.biblio.udg.mx:2048/docview/304393522?accountid=289 15

Adinkrah, M. (2012). Better dead than dishonored: Masculinity and male suicidal behavior in contemporary Ghana. Social Science \& Medicine, 74(4), 474-481. doi:10.1016/j.socscimed.2010.10.011

Agudelo, D., Bretón-López, J., \& Buela-Casal, G. (2003). Análisis bibliométrico de las revistas de Psicología Clínica editadas en castellano. Psicothema, 15(4), 507-616.

Anderson, E., Adams, A., \& Rivers, I. (2012). "I Kiss Them Because I Love Them": The Emergence of Heterosexual Men Kissing in British Institutes of Education. Archives of Sexual Behavior, 41(2), 421-430. doi:10.1007/s10508-010-9678-0

Annambhotla, K. (2000). Social information processing and emotion in hypermasculine men after rejection by a woman. (9976651 Ph.D.), Northern Illinois University, Ann Arbor. Retrieved from http://wdg.biblio.udg.mx:2048/docview/304633569?accountid=289 15

Apesoa-Varano, E. C., Barker, J. C., Unutzer, J., Aguilar-Gaxiola, S., Johnson, M. D., Tran, C., Hinton, L. (2015). Idioms of Distress Among Depressed White-Non-Mexican and Mexican-Origin Older Men. Journal of Cross-Cultural Gerontology, 30(3), 305-318. doi:10.1007/s10823-015-9267-8 
Arroyo Rueda, M. C., \& Soto Alanís, L. E. (2013). La dimensión emocional del cuidado en la vejez: la mirada de los adultos mayores. Cuadernos de Trabajo Social, 26, 337+.

Asakura, H., \& Torres Falcón, M. (2012). Género y vulnerabilidad extrema: migración centroamericana y trata de personas. In P. Ravelo Blancas \& H. Domínguez Ruvalcaba (Eds.), Diálogos Interdisciplinarios sobre Violencia Sexual (pp. 163-188): FONCA / Ediciones EÓN.

Backus, F. R. (2013). The relationship between cognitive emotion regulation and clinical symptoms: A gendered analysis. (3557742 Ph.D.), Boston College, Ann Arbor. Retrieved from http://wdg.biblio.udg.mx:2048/docview/1346228793?accountid=28 915

Barrientos Delgado, J., Salinas Meruane, P., Rojas Varas, P., \& Meza Opazo, P. (2011). Gender relations and masculinity in northern Chile mining areas: Ethnography in schoperías. Etnográfica. Revista do Centro em Rede de Investigação em Antropologia, 15(3), 413-440.

Bennett, K. M. (2007). "No Sissy Stuff": Towards a theory of masculinity and emotional expression in older widowed men. Journal of Aging Studies, 21(4), 347-356. doi:10.1016/j.jaging.2007.05.002

Bericat Alastuey, E. (2014). Matrimonio, desigualdad de género y bienestar socioemocional de los miembros de la pareja. In A. García Andrade \& O. Sabido Ramos (Eds.), Cuerpo y afectividad en la sociedad contemporánea. Algunas rutas del amor y la experiencia sensible en las ciencia sociales (pp. 191-228). México, D. F.: Universidad Autónoma Metropolitana-Unidad Azcapotzalco.

Bernard HR. (1995). Research Methods in Anthropology. London: Sage Publications.

Birenbaum-Carmeli, D., \& Inhorn, M. C. (2009). Masculinity and marginality: Palestinian men's struggles with infertility in Israel and Lebanon. JMEWS: Journal of Middle East Women's Studies, 5, 23+. Retrieved from https://muse.jhu.edu/article/262354

Borges, A. L. V., \& Schor, N. (2007). Homens adolescentes e vida sexual: heterogeneidades nas motivações que cercam a iniciação sexual Adolescent males and sex life: heterogeneous motivations. 
Cadernos de Saúde Pública, 23(1), 225-234. doi:

http://dx.doi.org/10.1590/S0102-311X2007000100024

Bourdieu, P. (2000). La dominación masculina. Barcelona: Editorial Anagrama.

Brandes, S. (2004). Drink, Abstinence, and Male Identity in Mexico City.

In M. Gutmann (Ed.), Changing men and masculinities in Latin America (pp. 153-178). United States: Duke University Press. Branney, P., Witty, K., Braybrook, D., Bullen, K., White, A., \& Eardley, I. (2014). Masculinities, humour and care for penile cancer: a qualitative study. Journal of Advanced Nursing, 70(9), 2051-2060. doi:10.1111/jan.12363

Brenton, J., \& Elliott, S. (2014). Undoing gender? The case of complementary and alternative medicine. Sociology of health \& illness, 36(1), 91-107. doi: 10.1111/1467-9566.12043

Brownhill, S., Wilhelm, K., Barclay, L., \& Schmied, V. (2005). 'Big build': hidden depression in men. Australian \& New Zealand Journal of Psychiatry, 39(10), 921-931. doi:10.1111/j.14401614.2005.01665.x

Brussoni, M., Creighton, G., Olsen, L. L., \& Oliffe, J. L. (2013). Men on Fathering in the Context of Children's Unintentional Injury Prevention. American Journal of Mens Health, 7(1), 77-86. doi: $10.1177 / 1557988312462739$

Bryant, L., \& Garnham, B. (2014). Economies, ethics and emotions: Farmer distress within the moral economy of agribusiness. Journal of Rural Studies, 34, 304-312. doi:10.1016/j.jrurstud.2014.03.006 Bueso-Izquierdo, N., Hidalgo-Ruzzante, N., Burneo-Garcés, C., \& PérezGarcía, M. (2015). Procesamiento emocional en maltratadores de género mediante el Test de Expresiones Faciales de Ekman y la Tarea Stroop Emocional. Revista Latinoamericana de Psicología, 47, 102+. doi: 10.1016/j.rlp.2015.02.001

Buzzanell, P. M., \& Turner, L. H. (2003). Emotion Work Revealed by Job Loss Discourse: Backgrounding-Foregrounding of Feelings, Construction of Normalcy, and (Re)instituting of Traditional Masculinities. Journal of Applied Communication Research, 31(1), 27. doi: http://dx.doi.org/10.1080/00909880305375 
Canham, S. L. (2009). The interaction of masculinity and control and its impact on the experience of suffering for an older man. Journal of Aging Studies, 23(2), 90-96. doi: 10.1016/j.jaging.2008.12.003

Cano Rodas, A. M., Motta Ariza, M. E., Valderrama Tibocha, L. E., \& Gil Vargas, C. A. (2016). Jefatura masculina en hogares monoparentales: adaptaciones de los hombres a las necesidades de sus hijos. Revista colombiana de sociología, 39(1), 123-145. doi: https://doi.org/10.15446/rcs.v39n1.56344

Ceballos Fernandez, M. (2012). Indicadores aplicados a la visión dominante de la masculinidad por adolescentes de educación secundaria: la importancia del" deber ser" hombre. Ultima década(36), 141. doi: http://dx.doi.org/10.4067/S071822362012000100007

Cleary, A. (2012). Suicidal action, emotional expression, and the performance of masculinities. Social Science \& Medicine, 74(4), 498-505. doi: 10.1016/j.socscimed.2011.08.002

Clifton, J. (2012). Birth fathers and their adopted children: fighting, withdrawing or connecting. Adoption \& Fostering, 36, 43-56. doi: https://doi.org/10.1177/030857591203600205

Coleman, P. T., Goldman, J. S., \& Kugler, K. (2009). Emotional intractability: Gender, anger, aggression and rumination in conflict. International Journal of Conflict Management, 20(2), 113-131. doi: https://doi.org/10.1108/10444060910949595

Conejero, S., Etxebarria, I., \& Montero, I. (2014). Gender differences in emotions, forgiveness and tolerance in relation to political violence. Spanish Journal of Psychology, 17. doi: 10.1017/sjp.2014.9

Connell, R. W. (2003). Maculinidades. México: PUEG.

Cottingham, M. D. (2013). Men who care: How organizations and individuals negotiate masculinity, emotional capital, and emotion practice in nursing. (3671072 Ph.D.), The University of Akron, Ann Arbor. Retrieved from

http://wdg.biblio.udg.mx:2048/docview/1645957480?accountid=28 915

Cottingham, M. D. (2015). Learning to "Deal" and "De-escalate": How Men in Nursing Manage Self and Patient Emotions. Sociological Inquiry, 85(1), 75-99. doi:10.1111/soin.12064 
Creswell, J. W. (2012). Qualitative inquiry and research design: Choosing among five approaches. London: Sage publications.

Cruz Sierra, S. (2010). Performatividad e identidad en la experiencia de la intimidad en hombres jóvenes (Journal, Electronic). Retrieved from http://conricyt1.summon.serialssolutions.com/2.0.0/link/0/eLvHCX MwfV07T8MwED7RSAjEwqMIKEgWA1uTNs7DHIFFYQAEIIm lcuqLGpRHRdohv4ufwB_j7LRCYugW5azIkezP953vvgPgvjvo_8 MEiVxh5KMS6UwEoUhRJzqJ4iRWQvupKX--

n_DJG_94Cok3btJ2iSq5fiBc6fLAm9NJkSvvucI6q03knNwnjXYH ZKr91xzrxva2yzSZas9c9Xq0zYfSKysuvMhd6LQDHTEwPM01T KQF7YhYTVtZTQtHCtNG2VmVqjj4g87xIeyuncQj2MHyGLqtkk fDbpiRiVW2FW9zAq8v65x_0_-

BJsKQZbbq1j6XLFcMNzrGmWIazZuM7MVmxLwqiBDX7PPn2 0JeF67Hd--jh76Z03Qd2JmOHm8HQ6swIwQ5DqfglFWJZ8AIsJkxkUYJiqg00calbdUKumHWuvQP4felg9dbLX2YL9QDdRiEtw118rvII9Iu-E281y-At_9pMC

Cruz Sierra, S. (2011). Sentido y práctica de la intimidad masculina: Una mirada desde los hombres View. Sociológica (México), 26(73), 183-207. Retrieved from

http://www.scielo.org.mx/scielo.php?script=sci_arttext\&pid=S0187 $-01732011000200007$

Chili, S., \& Maharaj, P. (2015). 'Becoming a father': perspectives and experiences of young men in Durban, South Africa. South African Review of Sociology, 46(3), 28-44. doi: http://dx.doi.org/10.1080/21528586.2015.1059775

Day, K., Stump, C., \& Carreon, D. (2003). Confrontation and loss of control: Masculinity and men's fear in public space. Journal of Environmental Psychology, 23(3), 311-322. doi:10.1016/s02724944(03)00024-0

de Oliveira Pimenta, S. M. (2012). Humano, demasiadamente humano: sobre emoçoes e masculinidade. D.E.L.T.A.(28:Especial), 605-637. doi: http://dx.doi.org/10.1590/S0102-44502012000300009

Duncombe, J., \& Marsden, D. (1993). Love and intimacy: The gender division of emotion and "emotion work": A Neglected Aspect of Sociological Discussion of Heterosexual Relationships. Sociology, 27(2), 221-241. 
Emslie, C., Ridge, D., Ziebland, S., \& Hunt, K. (2006). Men's accounts of depression: Reconstructing or resisting hegemonic masculinity?

Social Science \& Medicine, 62(9), 2246-2257.

doi:10.1016/j.socscimed.2005.10.017

Enríquez Rosas, R. (2009). El crisol de la pobreza. Mujeres, subjetividades, emociones y redes sociales. Guadalajara: ITESO.

Escorcia Otalora, T. A. (2008). El análisis bibliométrico como herramienta para el seguimiento de publicaciones científicas, tesis y trabajos de grado. (Licenciatura), Pontificia Universidad Javeriana, Bogotá, D. C. Retrieved from http://www.javeriana.edu.co/biblos/tesis/ciencias/tesis209.pdf

Evans, M. (2012). Feeling my way: emotions and empathy in geographic research with fathers in Valparaiso, Chile. Area, 44(4), 503-509. doi:10.1111/j.1475-4762.2012.01104.x

Evers, C. (2009). 'The Point': surfing, geography and a sensual life of men and masculinity on the Gold Coast, Australia. Social \& Cultural Geography, 10(8), 893-908. doi:10.1080/14649360903305783

Gaia, A. C. (2013). The role of gender stereotypes in the social acceptability of the expression of intimacy. Social Science Journal, 50(4), 591-602. doi:10.1016/j.soscij.2013.08.006

Galasinski, D. (2004). Men and the language of emotions (Primera ed.). New York: Palgrave McMillan.

González de Dios, J., Moya, M., \& Mateos Hernández, M. A. (1997). Indicadores bibliométricos: características y limitaciones en el análisis de la actividad científica. An Esp Pediatr, 47(3), 235-244. Retrieved from https://www.aeped.es/sites/default/files/anales/473-3.pdf

Gordon, S. L. (1990). Social structural effects on emotions. In T. D. Kemper (Ed.), Research agendas in the sociology of emotions (pp. 145-179). USA: State University Of New York Press.

Green, G., Emslie, C., O'Neill, D., Hunt, K., \& Walker, S. (2010). Exploring the ambiguities of masculinity in accounts of emotional distress in the military among young exservicemen. Social Science \& Medicine, 71(8), 1480. doi: 10.1016/j.socscimed.2010.07.015

Green, J. D., \& Addis, M. E. (2012). Individual differences in masculine gender socialization as predictive of men's psychophysiological 
responses to negative affect. International Journal of Men's Health, 11, 63+. doi: 10.3149/jmh.1101.63

Gutmann, M. (Ed.) (2004). Changing Men and Masculinities in Latina America (2nd edition ed.). United States of America: Duke University Press.

Gutmann, M. C. (2000). Ser hombre de verdad en la Ciudad de México. Ni macho ni mandilón. México, D.F.: El Colegio de México. Hernández Sánchez, E. (2008). Entre la memoria y el olvido: padres migrantes indígenas. In J. C. Ramírez Rodríguez \& G. Uribe Vázquez (Eds.), Masculinidades: el juego de género de los hombres en el que participan las mujeres (pp. 201-216). México: Plaza y Valdés / Universidad de Guadalajara / UNFPA / AMEGH / AJC.

Holter, Ø. G. (2005). Social Theories for Researching Men and Masculinities. Direct Gender Hierarchy and Structural Inequality. In M. H. Kimmel, Jeff; Connell, R. W. (Ed.), Handbook of Studies on Men \& Masculinities (pp. 15-34). Thousand Oaks, London, New Delhi: Sage Publications.

Hochschild, A. R. (2003). The managed heart: comercialization of human feeling USA: The Regents of University of California.

Hochschild, A. R. (2007). Exploring the Managed Heart. In H. Wulff (Ed.), The emotions. A cultural reader (pp. 83-92). United Kingdom: Berg.

Jackson, B. A. (2012). Bonds of Brotherhood: Emotional and Social Support among College Black Men. The Annals of the American Academy of Political and Social Science, 642(1), 61-71. doi: https://doi.org/10.1177/0002716212438204

Jackson, B. A., \& Wingfield, A. H. (2013). Getting Angry to Get Ahead: Black College Men, Emotional Performance, and Encouraging Respectable Masculinity. Symbolic Interaction, 36(3), 275-292. doi:10.1002/symb.63

Javaloy Mazón, F. (1996). Hinchas violentos y exitación emocional. Revista de Psicología del Deporte, 5(2), 93-104.

Jimenez, L., \& Walkerdine, V. (2011). A psychosocial approach to shame, embarrassment and melancholia amongst unemployed 
young men and their fathers. Gender \& Education, 23(2), 185-199. doi:10.1080/09540253.2010.490202

Johnston, M. S. (2016). Men can change: transformation, agency, ethics and closure during critical dialogue in interviews. Qualitative Research, 16(2), 131-150. doi:10.1177/1468794115569561

Kaplan, D. (2007). Folk Models of Dyadic Male Bonds in Israeli Culture. The Sociological Quarterly, 48(1), 47-72. doi: 10.1111/j.15338525.2007.00070.x

Kaufman, M. (1997). Las experiencias contradictorias del poder entre los hombres. In T. Valdés \& J. Olavarría (Eds.), Masculinidad/es (pp. 63-81). Santiago de Chile: Isis Internacional / Flacso - Chile.

Keddie, A. (2006). Fighting, anger, frustration and tears: Matthew's story of hegemonic masculinity. Oxford Review of Education, 32(4), 521-534. doi:10.1080/03054980600884243

Kimmel, M. (1997). Homofobia, temor, vergüenza y silencio en la identidad masculina. In T. Valdés \& J. Olavarría (Eds.), Masculinidad/es (pp. 49-62). Santiago de Chile: Isis Internacional / Flacso - Chile.

Kimmel, M.H., Hearn, J., Connell, R. W. (Ed.) (2005). Handbook of Studies on Men \& Masculinities. Thousand Oaks, London, New Delhi: Sage Publications.

Kobelinsky, C. (2012). Ver o no ver al refugiado. La evaluación de las solicitudes de asilo (por motivos sexuales) en Francia. Revista Temas de Antropología y Migración(No. 4, Diciembre), 13-29. Le Breton, D. (2009). Las pasiones ordinarias. Antropología de las emociones. Buenos Aires: Nueva Visión.

Lively, K. (2008). Emotional Segues and the Management of Emotion by Women and Men. Social Forces, 87(2), 911-936. doi:

https://doi.org/10.1353/sof.0.0133

Lomas, T., Cartwright, T., Edginton, T., \& Ridge, D. (2013). 'I was so done in that I just recognized it very plainly, "You need to do something"': Men's narratives of struggle, distress and turning to meditation. Health, 17(2), 191.

Lutz, C., \& White, M. (1986). The anthropology of emotions. Annual Review of Anthropology, 15, 405-436. 
Lutz, C. A. (1998). Unnatural Emotions: Everyday sentiments on a micronesian atoll and their challenge to western theory. Chicago: The University of Chicago Press.

Mann, R., Tarrant, A., \& Leeson, G. W. (2015). Grandfatherhood: Shifting Masculinities in Later Life. Sociology, 50(3), 594. doi: $10.1177 / 0038038515572586$

Marquis Currier, D. (2004). Gendered athletes: The social construction of gender, sexuality, and emotion among college athletes. (Doctor of Philosophy), University of Connecticut.

Marsh, K., \& Musson, G. (2008). Men at Work and at Home: Managing Emotion in Telework. Gender, Work \& Organization, 15(1), 31-48. doi:10.1111/j.1468-0432.2007.00353.x

Martín Cabello, A. (2011). Construyendo la masculinidad: fúbol, violencia e identidad. RIPS. Revista de Investigaciones Políticas y Sociológicas, 10(2), 73-95. Retrieved from http://www.redalyc.org/articulo.oa?id=38021386005

Martin, J. (2009). Relationships of power: Exploring teachers' emotions as experienced in interactions with their peers. (NR60728 Ph.D.), University of Victoria (Canada), Ann Arbor. Retrieved from http://wdg.biblio.udg.mx:2048/docview/504810846?accountid=289 15

McGill, O. D. (2010). The Influence of Masculinity on Restrictive Emotionality Among African American Men. (3428416 Ph.D.), Walden University, Ann Arbor. Retrieved from http://wdg.biblio.udg.mx:2048/docview/818725106?accountid=289 15

Menéndez, E. (1990). Morir de alcohol: saber y hegemonía médica. México: Alianza.

Meth, P. (2009). Marginalised men's emotions: Politics and place. Geoforum, 40(5), 853-863. doi:10.1016/j.geoforum.2009.07.002 Montes, V. (2013). The Role of Emotions in the Construction of Masculinity: Guatemalan Migrant Men, Transnational Migration, and Family Relations. Gender \& Society, 27(4), 469-490. doi:10.1177/0891243212470491

Moraes, D. C. A., Oliveira, R. C., \& Costa, S. F. G. (2014). Adesão de homens vivendo com HIV/Aids ao tratamento antirretroviral 
antirretroviral. Escola Anna Nery, 18(4), 676-681. doi:10.5935/1414-8145.20140096

Nash, J. E. (2012). Ringing the Chord: Sentimentality and Nostalgia among Male Singers. Journal of Contemporary Ethnography, 41(5), 581-606. doi:10.1177/0891241611429943

Nixon, D. (2009). 'I Can't Put a Smiley Face On': Working-Class Masculinity, Emotional Labour and Service Work in the 'New Economy’. Gender, Work \& Organization, 16(3), 300-322. doi:10.1111/j.1468-0432.2009.00446.x

Ospina Botero, M. (2007). Representaciones sociales de masculinidad y su expresion en el ambito familiar. Paginas de la UCPR, 69+.

Owen-Pugh, V., \& Allen, J. (2012). Accentuating the positive: The gendered identities of male problem-drinkers, and the questions these pose for the counselling profession. Counselling and Psychotherapy Research, 12(4), 267-275. doi:10.1080/14733145.2012.663777

Ramírez Rodríguez, J. C. (2010). Violencias y jóvenes. Enclaves de la masculinidad. In R. Reguillo (Ed.), Los jóvenes en México (pp. 359-394). México: Fondo de Cultura Económica; Consejo Nacional para la Cultura y las Artes.

Ramírez Rodríguez, J. C., \& Cervantes Ríos, J. C. (Eds.). (2013). Los hombres en México: veredas recorridas y por andar. Una mirada a los estudios de género de los hombres, las masculinidades. Guadalajara, México: Universidad de Guadalajara / AMEGH / Página Seis.

Randell, E., Jerdén, L., Öhman, A., Starrin, B., \& Flacking, R. (2015).

Tough, sensitive and sincere: how adolescent boys manage masculinities and emotions. International Journal of Adolescence and Youth, 21(4), 486-498. doi: http://dx.doi.org/10.1080/02673843.2015.1106414

Rodriguez-Ramirez, H. (2008). The social construction of the masculinities and its articulation in the emotions. (3314528 Ph.D.), University of Puerto Rico, Rio Piedras (Puerto Rico), Ann Arbor. Retrieved from http://wdg.biblio.udg.mx:2048/docview/304401836?accountid=289 15 
Romaní, F., Huaman, C., \& González Alcalde, G. (2011). Estudios bibliométricos como línea de investigación en las ciencias biomédicas: una aproximación para el pregrado. CIMEL Ciencia e Investigación Médica Estudiantil Latinoamericana, 16(1), 52-62. Retrieved from http://www.redalyc.org/articulo.oa?id=71723602008

Rumsey, S. (2008). How to find information. A guide for researchers. Berkshire: McGraw, Hill Open University Press.

Ruspini, E., Hearn, J., Pease, B. \& Pringle, K. (2011). Men and Masculinities Around the world. New York, EUA: Palgrave Macmillan.

Sabo, D. (2005). The study of masculinities and men's health. In M. H. Kimmel, Jeff; Connell, R. W. (Ed.), Handbook of Studies on Men \& Masculinities (pp. 326-352). Thousand Oaks, London, New Delhi: Sage Publications.

Seal, D. W., \& Ehrhardt, A. A. (2003). Masculinity and urban men: perceived scripts for courtship, romantic, and sexual interactions with women. Culture Health \& Sexuality, 5(4), 295-319. doi:10.1080/136910501171698

Seidler, V. (2007). Masculinities, Bodies and Emotional Life. Men and Masculinities, 10(1), 9-21. doi: https://doi.org/10.1177/1097184X07299636

Seidler, V. J. (2000). La sinrazón masculina. Masculinidad y teoría social. México: Paidós / UNAM-PUEG / CIESAS.

Shelley, M. T. (2007). Take it like a man: A study of men's emotion culture. (3442251 Ph.D.), Vanderbilt University, Ann Arbor. Retrieved from http://wdg.biblio.udg.mx:2048/docview/851892202?accountid=289 15.

Shields, S. A., Garner, D. N., Di Leone, B., \& Hadley, A. M. (2007). Gender and emotion. In J. E. Stets \& J. H. Turner (Eds.), Handbook of the sociology of emotions (pp. 63-86). New York: Springer.

Spraggins, J. D. (1995). Body and soul: The sociology of the bodies and emotions of male gun owners. (9542962 Ph.D.), University of Michigan, Ann Arbor. Retrieved from 


\section{http://wdg.biblio.udg.mx:2048/docview/304206662?accountid=289 15}

Thagaard, T. (1997). Gender, Power, and Love: A Study of Interaction between Spouses. Acta Sociologica, 40(4), 357-376.

doi:10.1177/000169939704000402

Thomeer, M. B., Reczek, C., \& Umberson, D. (2015). Gendered emotion work around physical health problems in mid- and later-life marriages. Journal of Aging Studies, 32, 12-22. doi:10.1016/j.jaging.2014.12.001

Treviño Siller, S., Villanueva Borbolla, M., Marcelino Sandoval, Y., \& Álvarez Guillén, F. (2014). Masculinidad, accidentes viales y políticas públicas. In J. G. Figueroa (Ed.), Políticas públicas y la experiencia de ser hombre. Paternidad, espacios laborales, salud y educación (pp. 209-245). México: El Colegio de México.

Turner, J. H., \& Stets, J. E. (2009). The sociology of emotions. New York: Cambridge University Prees.

Umberson, D., Anderson, K. L., Williams, K., \& Chen, M. D. (2003). Relationship dynamics, emotion state, and domestic violence: A stress and masculinities perspective. Journal of Marriage and Family, 65(1), 233-247. doi:10.1111/j.1741-3737.2003.00233.x

Vaccaro, C. A., Schrock, D. P., \& McCabe, J. M. (2011). Managing Emotional Manhood: Fighting and Fostering Fear in Mixed Martial Arts. Social Psychology Quarterly, 74(4), 414-437. doi: $10.1177 / 0190272511415554$

Villa, J. (2015). Body, masculinity and style in the upper-class youth of Lima. Debates En Sociologia(40), 61-91.

Waitt, G., \& Stanes, E. (2015). Sweating bodies: Men, masculinities, affect, emotion. Geoforum, 59, 30-38. doi:10.1016/j.geoforum.2014.12.001

Walton, C., Coyle, A., \& Lyons, E. (2004). Death and football: An analysis of men's talk about emotions. British Journal of Social Psychology, 43(3), 401-416. doi:10.1348/0144666042038024

Wentzell, E. (2014). Masculinity and emotion in Mexican men's understandings of erectile dysfunction aetiology and treatment. Culture Health \& Sexuality, 16(2), 164-177. doi:10.1080/13691058.2013.854409 
White, A. M., \& Peretz, T. (2010). Emotions and Redefining Black Masculinity Movement Narratives of Two Profeminist Organizers. Men and Masculinities, 12(4), 403-424. doi:10.1177/1097184x08326007

Wilkins, A. (2012). "Not Out to Start a Revolution": Race, Gender, and Emotional Restraint among Black University Men. Journal of Contemporary Ethnography, 41(1), 34-65. doi: $10.1177 / 0891241611433053$

Winlow, S., \& Hall, S. (2009). Retaliate first: Memory, humiliation and male violence. Crime Media Culture, 5(3), 285-304. doi:10.1177/1741659009349243

Wu, T. W., Oliffe, J. L., Bungay, V., \& Johnson, J. L. (2015). Male ICU Nurses' Experiences of Taking Care of Dying Patients and Their Families: A Gender Analysis. American Journal of Mens Health, 9(1), 44-52. doi:10.1177/1557988314528236

Zackariasson, M. (2009). Angry young men? Masculinities and emotion among young male activists in the global justice movement. The Journal of Men's Studies, 17, 31-46. doi:

https://doi.org/10.3149/jms.1701.31

Juan Carlos Ramírez Rodríguez es Profesor Investigador en el Programa Interdisciplinario de Estudios de Género (PIEGE). Departamento de Estudios Regionales - CUCEA. Universidad de Guadalajara, México.

María del Pilar Gómez González es doctoranda del Programa de Doctorado en Ciencias de la Salud Pública. Departamento de Salud Pública. Centro Universitario de Ciencias de la Salud. Universidad de Guadalajara, México.

Norma Celina Gutiérrez de la Torre es Directora del Centro de Estudios de Población. Coordinadora del Programa Interdisciplinario de Estudios de Género (PIEGE). Departamento de Estudios Regionales - CUCEA, Universidad de Guadalajara, México

Marcela Viridiana Sucilla Rodríguez es Maestrante Programa de Maestría en Gestión y Desarrollo Social. Departamento de Desarrollo Social. Centro Universitario de Ciencias Sociales y Humanidades. Universidad de Guadalajara, México

Dirección de Contacto: Correspondencia directa a Juan Carlos Ramírez Rodríguez, Dpto. de Estudios Regionales - INESER, CUCEA Periférico Norte, 799, Núcleo Los Belenes, 45100, Zapopan, Jalisco (México), email: jucarlosra@ gmail.com 
Criterios de búsqueda de referencias bibliográficas para cada una de las bases de datos consultadas.

\begin{tabular}{ll}
\hline Base de datos & Criterio utilizado \\
\hline Emmerald & Keyword / Anywhere / Abstract \\
& All content \\
& Accepted articles \\
& Backfiles \\
& Articles and Chapters \\
& All dates \\
& Palabra clave / Documento completo \\
Global Issues in & Full Text Documents \\
Context & Peer Reviewed Journals \\
& Fecha de publicación: Todas \\
& Academic Journals \\
& Documentos de texto completo \\
Informe Académico & Periódicos revisados por pares \\
& Todas las fechas \\
& Palabra clave / Documento completo \\
& Article title / Keywords or abstract / Article full text \\
Ingenta connet & All content \\
& Item Title / Abstract / Full Text \\
Jstor & Articles \\
& Búsqueda básica \\
Web of Science & Todas las bases de datos \\
& Tema \\
& Tipos de documento: articles \\
Scielo & Advanced search \\
& Keywords \\
Wiley & Journals \\
& All fields (para los términos “parte del discurso") \\
& Buscar documentos \\
& Artículos de revista \\
Dialnet & Tesis \\
& Túsqueda de artículos \\
& Todos los índices \\
&
\end{tabular}


Anexo 1

Continuación

\begin{tabular}{|c|c|}
\hline Base de datos & Criterio utilizado \\
\hline \multirow[t]{8}{*}{ Consorcio } & Búsqueda avanzada \\
\hline & Términos temáticos \\
\hline & Artículo de revista/Articulo de la publicación \\
\hline & especializada/ Revista / eJournal \\
\hline & Texto completo en línea \\
\hline & Recursos arbitrados, incluye los revisados por pares \\
\hline & Excluir reseñas de libros y artículos de prensa \\
\hline & Tipo de documento: artículo de revista. \\
\hline \multirow[t]{4}{*}{ ERIC } & Advance search \\
\hline & peer reviewed only \\
\hline & Full text available on ERIC \\
\hline & Journal articles \\
\hline Lippincott Williams \& & Keywords \\
\hline \multirow[t]{3}{*}{ Wolk (Ovid) } & All the numbers \\
\hline & Summary \\
\hline & Text complete \\
\hline \multirow[t]{4}{*}{ Proquest } & Palabras clave \\
\hline & Búsqueda avanzada \\
\hline & Texto completo \\
\hline & Evaluado por expertos \\
\hline \multirow[t]{4}{*}{ Science Direct } & Palabras clave \\
\hline & Búsqueda avanzada \\
\hline & Journals / Artículo/Ciencias Sociales \\
\hline & Todos los años \\
\hline \multirow[t]{4}{*}{ Scopus } & Palabras clave \\
\hline & Búsqueda avanzada \\
\hline & Artículos \\
\hline & Ciencias Sociales \\
\hline \multirow{5}{*}{$\begin{array}{l}\text { Academic one file } \\
\text { unique }\end{array}$} & Búsqueda avanzada \\
\hline & Palabra clave \\
\hline & Documentos de texto completos \\
\hline & Periódicos revisados por pares \\
\hline & Todas las fechas \\
\hline
\end{tabular}


256 Ramírez Rodríguez et al. - Masculinidades y Emociones

Anexo 1

Continuación

\begin{tabular}{ll}
\hline Base de datos & Criterio utilizado \\
\hline Annual reviews & Advanced search \\
& Keywords \\
& Journals (solo acepta palabras en inglés) \\
Cambridge Collection & $\begin{array}{l}\text { Advanced search } \\
\text { Search all journals and book content } \\
\text { Keywords } \\
\text { Search exact phrase }\end{array}$ \\
& Búsqueda avanzada \\
Ebsco & Términos temáticos \\
& Texto completo \\
& Publicaciones académicas (arbitradas) \\
& Booleano / frase \\
& Todos / todas \\
\hline
\end{tabular}

Fuente. Elaboración propia considerando información de las bases de datos consultadas. 


\section{Hipatia Press \\ www.hipatiapress.com}

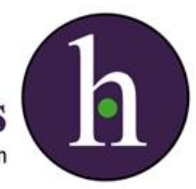

Instructions for authors, subscriptions and further details:

http://mcs.hipatiapress.com

\section{Analysing Masculinity from the Key Theoretical Lenses and Searching for Linkages with Violence against Women}

Anisur Rahman Khan \& Shahriar Khandaker ${ }^{1}$

1) East West University, Bangladesh

Date of publication: October $21^{\text {st }}, 2017$

Edition period: February 2018 - June 2018

To cite this article: Khan, A. R., \& Khandaker, S. (2017). Analysing Masculinity from the Key Theoretical Lenses and Searching for Linkages with Violence against Women. Masculinities and Social Change,6(3),257287. doi: 10.17583/MCS.2017.2593

To link this article: http://doi.org/10.17583/MCS.2017.2593

PLEASE SCROLL DOWN FOR ARTICLE

The terms and conditions of use are related to the Open Journal System and to Creative Commons Attribution License (CC-BY). 


\title{
Analysing Masculinity from the Key Theoretical Lenses and Searching for Linkages with Violence against
}

\section{Women}

\author{
Anisur Rahman Khan \& Shahriar Khandaker \\ East West University
}

\section{Abstract}

In recent years, masculinity has become both an important and influential academic discourse in the domain of gender studies. Despite having been explained and theorised from many perspectives, it lacks overall clarity and varies widely across different social and cultural contexts. Keeping that perspective in mind, this study drew on a rigorous review of the literature and reflexive analysis has synthesised prominent and pertinent theoretical issues concerning masculinity with the objective of having a succinct as well as a methodical understanding of masculinity. This study also aims at developing the linkage between masculinity and violence against women. In spite of being viewed as a cause of violence against women; theoretical notions of masculinity and its relation to violence against women remain largely understudied. Overall, the findings of the study confirm that masculinity as a concept is expressed through certain socially accepted ideologies and practices and there are at least three major theoretical developments concerning masculinity. When applied, each of these theories can individually stand as a reason for violence against women. The nexus between masculinity and violence against women is very proximate, and masculinity appears to be a predominating force for perpetuating violence against women. Nonetheless, further wider empirical studies on masculinity and its relation to violence against women can draw new insights and understandings.

Keywords: Masculinity, psychoanalytical theory, sex role theory, hegemonic masculinity, violence against women 


\title{
Analizando la Masculinidad desde la Óptica Teórica y Buscando Vínculos con la Violencia de Género
}

\author{
Anisur Rahman Khan \& Shahriar Khandaker \\ East West University
}

\section{Resumen}

En los últimos años, la masculinidad se ha convertido en un importante e influyente discurso académico en el campo de los estudios de género. A pesar de haber sido explicado y teorizado desde muchas perspectivas, carece de claridad general y varía ampliamente en diferentes contextos sociales y culturales. Teniendo esta perspectiva en mente, este estudio se basó en una revisión rigurosa de la literatura así como el análisis reflexivo sobre cuestiones teóricas importantes y pertinentes relativas a la masculinidad con el objetivo de tener una comprensión clara y metódica de la masculinidad. Este estudio también tiene como objetivo profundizar sobre el vínculo entre la masculinidad y la violencia contra las mujeres. A pesar de ser vista como una causa de violencia contra las mujeres; las nociones teóricas de la masculinidad y su relación con la violencia contra las mujeres siguen siendo ampliamente estudiadas. En conjunto, los resultados del estudio confirman que la masculinidad como concepto se expresa a través de ciertas ideologías y prácticas socialmente aceptadas y hay por lo menos tres desarrollos teóricos importantes concernientes a la masculinidad. Cuando se aplican, cada una de estas teorías puede ser individualmente una razón para la violencia contra las mujeres. El nexo entre la masculinidad y la violencia contra las mujeres es muy próximo y la masculinidad parece ser una fuerza muy dominante para perpetuar la violencia contra las mujeres. Sin embargo, otros estudios empíricos más amplios sobre la masculinidad y su relación con la violencia contra las mujeres pueden traer nuevas aportaciones al tema.

Palabras clave: Masculinidad, teoría psicoanálitca, teoría del rol sexual, masculinidad hegemónica, violencia contra las mujeres 


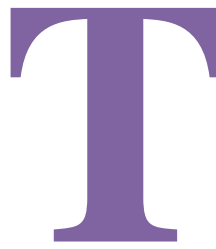

he word masculinity is derived from the Middle English masculine and Latin masulinus, which means 'male', 'of masculine gender', or 'male person'. It was used in these contexts since the late fourteenth-century. Later on, from the seventeenth century onwards it was further began to be used as 'appropriate' qualities of the male sex such as 'powerfulness', 'physicality', 'manliness' and 'virility' which have been extended in referring to traits or characteristics traditionally thought of suitable for men and showing maleness, manliness or manhood (Mangan \& Walvin, 1987 cited in Hearn, 2007, p. 390). Accordingly, in traditional understanding masculinity is associated with dominance, aggression, assertiveness, self-assurance and male characteristics like household head and breadwinner (Boonzaier \& Rey, 2003, p. 1020). Although such traits were treated as the constituents of an idealised version of masculinity, there was no absolute and concrete definition or standard of what is being meant by men and what standards are to be followed by men to be treated as real masculine. Until today, the notion regarding masculinity has always been subject to change and varies within and across cultures, social groupings and classes. There is nothing like modern masculinity or a set of determined standard of masculinity (Whitehead, 2002, p. 15-16), and what is being meant by masculine is likely to vary among various racial, ethnic, religious groups, social classes, age groups as well as among people with different sexual orientation and by geographical region (Fischer \& Good, 1998, p. 372).

The meanings of masculinity are constantly being changed, and its implications are always subject to be proved, and once proved, it is again questioned and has to be proved again (Kimmel, 1994, p. 122). Masculinity is in no way a fixed entity embedded only in body or personality traits of individuals, it is rather accomplished in social action and differs according to gender relations in a particular social and cultural setting (Connell \& Messerschmidit, 2005, p. 835). It is evident that a growing body of research on masculinity is now available, but the conceptual ambiguity of the term still exists till date (Good, Borst, \& Wallace, 1994, p. 3). The term masculinity is being used in a variety of ways, and it is one of those terms, which are endlessly being debated. It is applied to be defined as values, ideologies, experiences and meanings that are natural for a man or required 
for being real or a proper man in a particular cultural context (Flood, 2002, p. 204). 'Real man' is seen as being able to gratify his sexual needs and controls women. Moreover, he is the protector of women and children; he is the provider, the head of household (Abbott, 2000, p. 1312) and the breadwinner in the family (Boonzaier \& Rey, 2003, p. 1020). However, it does not necessarily mean that there are certain human qualities, which are inevitably or inherently masculine since maleness or masculinity might mean different things and might have different meanings to different people and groups (Hoffman, Hattie, \& Borders, 2005, p. 76). In general, masculinity refers to certain physical, behavioural and attitudinal qualities that are essential to be a man in a particular historical and cultural context (Mason-Grant, 2000, p. 322). It contains multiple images, behaviours, identities and views, which are often competing, and contradictory and the meanings of masculinity vary in the particular context, culture and time (Cornwall \& Lindisfarne, 1994, p. 12).

Masculinity is also a reaction against passivity, powerlessness and repression against all desires and traits that are treated negatively in a particular society (Kaufman, 1987, p. 11). The notion 'woman' has been produced as the negative version of masculinity (Hollway, 1996 cited in Alvesson \& Billing, 1997, p. 84). Women are passive and dominated by men are the typical portrayals of femininity (Horowitz \& Kaufman, 1987, p. 86). More specifically, traits, meanings, images and values that are associated with women are defined as femininity. Masculinity is viewed as the antithesis of femininity. This notion of the antithesis of femininity is at the heart of contemporary and historical conceptions of manhood (Kimmel, 1994, p. 126). In these circumstances, the discussion of masculinity cannot be done in isolation rather it should and must be in accordance or comparison with femininity (Alvesson \& Billing, 1997, p. 84). It is, indeed, challenging to escape from using the term 'men', 'male' and 'masculinity' and 'women', 'female' and 'femininity' without developing a binary notion of gender (Threadgold, 1990 cited in Cornwall \& Lindisfarne, 1994, p. 12). The concept masculinity is meaningless without its feminine counterpart; both the concepts are supplementary and complementary of one another (Ahmed, 2006, p. 15). The concept masculinity does not exist without in contrast with femininity; therefore, culture, which does not treat or speak of the relation between men and women, does not have a concept of 
masculinity. The concept is, thus, inherently relational with femininity (Connell, 2002, p. 31). More specifically, gender relates to the classification of being masculine and feminine and also refers to how these are socially constructed and sustained (Reid \& Wormald, 1982 cited in Walczak, 1988, p. 26). Therefore, the term masculinity is fundamental to understanding gender relations and has significant social and political implications.

Customarily, masculinity symbolises how to become a man and how to become a master of women, and it is often stated that being violent is an accepted and dominant way of being a man. Thus, perpetrating violence is a method of demonstrating manhood or masculinity (Hearn, 1998, p. 37). Men, both in the past and present, have committed majority of the violent acts of the world. Therefore, violence, albeit violence against women, is meant to be a masculine act because there is very little femininity expressed in acts of violence (Edwards, 2006, p. 39). Masculinity, thus, provides the symbolic meaning of violence (Welzer-Lang, cited in Blanchet, Biswas, \& Lucky, 2001, p. 11). Nonetheless, men commit most violence against women, but it does not necessarily mean that all men are violent (Connell, 2000 cited in DeKeseredy \& Schwartz, 2005, p. 356).

Under the contextual premises mentioned above, this paper primarily makes a rigorous attempt to clarify the concept 'masculinity', and analyse as well as synchronise its key theoretical underpinnings. In addition to that, it also examines the possible interconnections between masculinity and violence against women. In preparing this paper, a rigorous review of the literature was sought, and researchers' own reflexive understanding was used in analysing the key problems and issues. This research answered two questions, firstly; what are the major theoretical developments about masculinity, and secondly; how these theoretical issues are associated with violence against women? The following analysis aims to provide directives for empirical researchers to undertake future project about masculinity and violence against women on a wider scale.

\section{Dominant Theories on Masculinity}

There are many different explanations and ideas ascribed to the term 'masculinity'. These statements and ideas are stemmed from many thoughts and insights of various scholars. In the following sections, the fundamental 
theoretical notions related to masculinity have been discussed, and some criticisms have also been produced.

\section{Freud's Psychoanalytical Theory}

The first attempt to make a scientific inquiry into masculinity on both possible and necessary senses was made by the great Austrian medical psychologist Sigmund Freud (1856-1939) in the late nineteenth century. Though Freud nowhere wrote a regular discussion of masculinity, it is one of his recurring themes. His idea regarding masculinity came in the initial statement of psychoanalytical principles, methods and declarations (Connell, 1995, p. 8). Psychoanalytical approach has predominantly focused on the nature and relations of masculinity and femininity by giving emphasis on sexuality, even though its insights have been extended to the analysis of numerous conceptions and phenomena such as; sexual difference, dream life, neurosis, hysteria, perversion and also the existence of the unconscious (Lafrance, 2004, p. 642-43).

Sigmund Freud developed the idea regarding masculinity and femininity in his psychoanalysis by exploring the attachment of boys and girls to their bodies and their relationships with their parents (Flanagan, 1982, p. 64); and particularly with a set of social experiences (Horowitz \& Kaufman, 1987, p. 86). Further to note, though fundamentally Freud conceives the idea 'Anatomy is Destiny', but he also holds the presupposition that everything is determined and shaped through a person's interface with social world (Ferrell, 2000, p. 404). Beginning with the body, Freud states that at the age of around four, boys and girls naturally explore their bodies. At this stage, boys start playing with their penises and girls with their clitorises. However, the autoerotic activity for both boys and girls is the same, and owing to this uniformity initially there is no possibility of a distinction between the two sexes (Flanagan, 1982, p. 64). They do not seem to have significant experiences of sexual desires or gratifications at this stage (Horowitz \& Kaufman, 1987, p. 86). Moreover, Freud describes the body as 'polymorphosuly perverse', capable of experiencing pleasure from any sensations (Ferrell, 2000, p. 404). His 'oedipus complex' (a concept based on the Greek myth of Oedipus) is instrumental to elucidate how a 'polymorphosuly perverse' is taken to the social world and to have a 
separate sexual identity (Ferrell, 2000, p. 404). Oedipus complex is the basis of his psychoanalysis and provides a very complex emotional state for a child that involves the desire for one parent and hatred for another. Freud believed little boys and girls develop a strong sense of attraction towards parents of their opposite sex and a strong jealously of their same sex parents (Flanagan, 1982, p. 64). For a boy, his father stands in his path as a source of fear. In his eyes, his father is bigger, stronger and more sexually potent. The fear father is the fear of 'castration' (loss of penis), and due to that fear, the boy puts aside his identification/sexual desire with his mother, which in turn, makes him capable of sexual union with a mother like replacement; i.e., with another woman. More specifically, through an oedipal state, a boy strives for a secure identity for himself as a man and becomes both masculine and heterosexual at the same time. According to this model, masculinity is inextricably tied to sexuality that resembles the sexuality of his father, which is often oppressive, possessive and punitive (Kimmel, 1994, p. 126-127). The fear of castration is powerful because patriarchal and heterosexual society creates a norm that without possessing a penis one cannot be powerful, active or a lover of a woman (Horowitz \& Kaufman, 1987, p. 86). Therefore, for Freud, the whole process is a formative moment in masculinity, and it produces a dynamics in a formative relationship (Connell, 1995, p. 9).

Freud, on the other hand, observes that the farewell to oedipal love for mother helps the boy to move beyond the triangular of family drama and develop a romantic attachment to more appropriate female objects. However, a girl, on the other hand, relinquishes her love towards her mother when she realises that she lacks a penis. This state is known as 'penis envy'. Due to 'penis envy' a girl starts feeling antipathy towards her mother and attraction towards her father (Lafrance, 2004, p. 643-44). Father takes the mother's place in a girl's eye because she knows that the father has what she wants or what she lacks; that is a penis. Such biologically destined genitals make a greater impact on all subsequent psycho-social developments. For example, girls feel highly wounded for not having penis and boys feel highly superior for having a penis (Flanagan, 1982, p. 66-67). It is further to note that, according to Freud, masculinity at a very early stage is a natural state for both the sexes but a girl retreats from masculinity to femininity once she discovers that she has no penis and it leads her to a 
very fateful and unhappy situation (Person, 2009, p. 5). However, Freud notes that the feminine condition is established in her only when her wish of having a penis is replaced by the desire of having a child (Friedan, 1963, p. 103). As a matter of fact, 'penis envy' paves the way to typical femininity for a girl while 'castration anxiety' opens the way to traditional masculinity for a boy (Bem, 1993, p. 58).

In feminist theory, the importance psychoanalysis developed by Freud is of great significance since his theory explains the structure of individual or self through the operation of sexual differences (Ferrell, 2000, p. 403). Freud's analysis of masculinity was remarkable since it opened a new window and provided a guiding concept for development of masculinity (Connell, 1995, p. 9). Although as a theory it is both paradoxical and controversial, but it was the first serious attempt and the most interesting one for explaining and analysing masculinity (Connell, 1994, p. 11; Kilmartin, 2004, p. 319). Judith Mitchell (1997 cited in Gardiner, 2002, p. 195) puts a value to psychoanalysis in the sense that it adequately explains the nature of masculinity, femininity, heterosexuality and the social organisation of gender. Its masculine biases should not be seen as a reason for rejecting it totally rather it should be treated as a cause for rethinking the concept and how to make best use of it in development of feminist scholarship (Brennan, 1992; Flax, 1990; Kofman, 1980; Schneider, 1980 cited in Whitford, 2000, p. 1686).

The theory of Freud on psychoanalysis was criticised in many ways by feminist scholars. For example; Thomas (2007, p. 515) finds psychoanalytic accounts as prolonged, complicated, imprecise and incomplete. Irigaray (1985 cited in Ferrell, 2000, p. 404) states that Freud failed to answer the riddle of female desire because he has focused or understood on only one sex (male); other is the negation or absence of this sex, and he has defined women as castrated men. Simone de Beauvoir (1949 cited in Gardiner, 2002, p. 195) criticises Freud for viewing men as only human and women as mutilated men and relegating them to the state of other concerning male self. Kate Millett (1969 cited in Bem, 1993, p. 61) also criticises him for producing confusions. Whitford (2000, p. 1686) states that psychoanalytical theory fosters the desire of men and its central concepts explicitly or implicitly consider women as inferior. Many feminists, therefore, - accuse Freud for coercingwomen in a double standard 
such as the typical woman is inferior, passive, masochistic and narcissistic but the woman who lacks these traits are neurotic, abnormal and masculine. These are undoubtedly negative traits attached with women (Gardiner, 2002, p. 195). According to Friedan (1963, p. 93), Freud is the prisoner of his own culture because much of his findings represent only the characteristics of certain middle-class European men and women. Radical feminists, on the other hand, view psychoanalysis as the agent of patriarchal culture and find its sexism contributing to violence against women (Gardiner, 2002, p. 195). According to Judith Butler (1990 cited in Whitford, 2000, p. 1686), psychoanalytical theory divides men and women by biologically determined sex, and in so doing, it has reinforced male primacy and made heterosexuality a cultural norm. Nevertheless, there is no evidence that the original gender is masculine as proposed by Freud. It is rather feminine proposed by Stroller (1968), and innate (both masculine \& feminine) offered by Hornby $(1924,1925,1932,1933)$ \& Jones (1948/1927, 1948/1933, 1984/1935 cited in Person, 2009, p. 5 \&10). Moreover, Hornby (1924 cited in Friedman \& Downey, 2002, p. 93) mainly blames Freudfor his misleading interpretation and states women do not feel inadequate because they do not have penis-these biologically determined feminine attributes are virtues, not defects. She further states that men are envious because of women's reproductive capacity.

Despite having many criticisms of Freud's theory of psychoanalysis, it still maintains a strong position in contemporary feminist theory (Gardiner, 2002, p. 199). Freud's psychoanalysis has at least provided a language to define women from an androcentric perspective (Bem, 1993, p. 62), and the distinction between a boy and a girl provides a symbolic meaning of masculinity and femininity (Giddens, 2001, p. 110). Lafrance (2004, p. 644), on the other hand, has provided some critical assumptions based on Freud's theory such as i) men's bodies are perfect and complete; ii) the presence and absence of male phallus/penis determines one's gender identity, sexual development and psychic formation, and iii) masculinity and femininity are based on patriarchal and heterosexual conception of normal sexuality-the only acceptable way of organising sexual congress. There is, thus, no way to fully discard Freud's theory of psychoanalysis in understanding masculinity. His analysis has heralded the theoretical development of the concept in the academia. 


\section{Sex Role Theory}

A significant attempt in social science in understanding masculinity has been due to the idea of 'sex role', a concept connected to the notion of sex differences. Being a man or being a woman is attached to one's sex-the 'sex role'. There are always two roles: male role and female role (Connell, 1995, p. 21-22). The term 'sex role' not only refers to societal norms regarding appropriate characteristics and behaviours for men and women (Serafica \& Rose, 1982, p. 11) but also indicates the social processes and practices associated with masculinity and femininity. The terms 'gender role' and 'sex role' are used interchangeably concerning internalisation of what is masculinity or femininity (Prentice, 2000, p. 223). Notably, the term 'sex role' is used more commonly and frequently than the term 'gender role' (Beere, 1990, p. 21; Turner, 1995, p. 1). Theoretically, the role is a process of learning, a process of socialisation and internalisation through which female character is produced by socialisation into female roles and male character into the male role (Connell, 1987, p. 48-49). Therefore, the relation between socialisation and sex role theory is very close, and the process of socialisation is often called 'role learning', 'role acquisition' or 'sex role socialisation' (Connell, 1987, p. 192). The basic idea of gender role theory is that in a given society men and women are socialised to learn different duties, responsibilities and rules of behaviour (Spence, Helmreich \& Sawin, 1980 cited in Martin \& Finn, 2010, p. 7). It is true that an individual is socialised in many different ways through different agents throughout his/her life such as school, neighbourhood, community, peergroup, workplace, religious institution but family is the most important institution to attain required roles for developing masculine and feminine personality characteristics (Maccoby, 1992 cited in Adams \& Coltrane, 2005, p. 233).

Sex role has been a critical theory in the study of masculinity and femininity since the 1970s. Over the years a good number of theories have given positive inputs in developing sex role theory, but it has its origin in 'Family, Socialisation and Interaction Process' of the work of sociologists Parsons \& Balles (1956). They have argued men and women are suited to different types of roles, and men are assigned to more instrumental roles while women take on more expressive roles. These roles are 
complementary to each other and are required to meet the functions of society. They have suggested that men and women have an obligation to be socialised to 'appropriate' roles (Pease, 2007, p. 554). Sex role theory suggests that a father must maintain authority in the family. His instrumental role model must demonstrate technical and executive expertise while mother's expressive model provides moral support to the family (Parsons \& Balles, 1956-, p. 51). The expressive model includes skills required to be emotional, sensitive, aware of other's feeling, interdependent and tender whereas instrumental model includes skills needed to be aggressive, independent, unemotional, assertive, strong and so on (Sahoo, 2004, p. 203). According to sex role principles, a mother maintains family's sense of taste and decorum. Any deviation from this role pattern may result in sex role dysfunction. If the socialisation or internalisation is perfect, a male child becomes an 'adequate technical performer' and 'cooperator' and a female child becomes a 'willing and accommodative person' (Parsons \& Balles, 1956/2002, p. 51). Focusing predominantly on socialisation process the early proponents of sex role theory have provided a level of understating on the course of being acquiring masculine and feminine roles in family and society.

In 1976, Brannon proposed an essential model called 'Blueprint of Masculinity' which is globally recognised as a role model on how to become masculine or how to attain certain necessary qualities of manhood. This model proposes four themes, which are treated as the benchmark for measuring masculinity (Kimmel, 1994, p. 126). The first idea of the model is No Sissy Stuff. This refers to the concept that a man should never do anything that is treated to be feminine. Masculinity is the complete rejection of femininity (Kimmel, 1994, p. 125; Pease, 2007, p. 554). This approach belongs to the idea that men and women are two different as well as opposite sexes (Furby, 1983 cited in Kahn, 2009, p. 56). This theme speaks of the stigma associated with feminine characteristics and qualities. Female features and qualities are highly objectionable or threatening to the role of masculinity (Harris, 1995, p. 133). The second theme is The Big Wheel. It refers to the idea that men must feel that they are in charge of the situations (Kahn, 2009, p. 56). Masculinity includes dominance and power over others, and it is expressed in success, wealth and status (Kimmel, 1994, p. 125). For example; masculinity correctly is represented in 
acquiring success and status in the breadwinning role (Pease, 2007, p. 554). The third theme is The Sturdy Oak. According to this theme, masculinity is an expression of toughness, confidence and self-reliance. This theme encourages boys to be tough and self-confident (Harris, 1995, p. 133-134). It also provokes that to be a man one has to shun all emotions and remain calm in crisis to show self-reliance (Franklin, 1988 cited in Kahn, 2009, p. 56; Kimmel, 1994, p. 125). The last theme is Give'em Hell. The sole idea of this theme is that to be masculine one has to be courageous and a risk taker (Kimmel, 1994, p. 126). A man subscribes to this issue in a variety of ways to attain masculine ideal (Kahn, 2009, p. 57). For example; this theme encourages a man to be aggressive, violent and daring in showing his masculinity (Harris, 1995, p. 133-134). Sex role theorists argue that these sorts of typical masculine notions are imposed on boys from birth and are reinforced through a different agent of socialisation (Farrell, 1975 cited in Pease, 2007, p. 554).

Inge Broverman who is one of the pioneers of sex role study in the early days of women's movement mentions that sex role definitions are implicitly incorporated into the self-image or self-concepts of both males and females (Mackie, 1980, p. 121). Sex role definitions point out the existence of highly consensus norms and values associated with the process of differentiation of men and women (Broverman et al., 1970 cited in Sahoo, 2004, p. 203). By researching male and female characteristics and their desirability Broverman et al. (1972 cited in Walczak, 1988, p. 29) find masculine traits are more desirable than female characters. It means that people expect men and women to behave very differently and they value male behaviour more highly as shown in the following manner. 
Table 1

Sex Roles

a) Masculine pole is more desirable

\begin{tabular}{ll} 
Feminine & Masculine \\
\hline Not at all aggressive & Very aggressive \\
Not at all independent & Very independent \\
Very emotional & Not at all emotional \\
Does not hide emotion at all & Almost always hides emotions \\
Very subjective & Very objective \\
Very easily influenced & Not at all easily influenced \\
Very submissive & Very dominant \\
Dislikes maths \& science very much & Likes maths \& science very much \\
Very excitable in a minor crisis & Not at all excitable in a minor crisis \\
Very passive & Very active \\
Not at all competitive & Very competitive \\
Very illogical & Very logical \\
Very home-oriented & Very worldly \\
Not at all skilled in business & Very skilled in business \\
Very sneaky & Very direct \\
Does not know the way of the world & Knows the way of the world \\
Feelings easily hurt & Feelings not easily hurt \\
Not at all adventurous & Very adventurous \\
Has difficulty making decisions & Can make decisions very easily \\
Cries very easily & Never crises \\
Almost never acts as a leader & Almost always acts as a leader \\
Not at all self-confident & Very self-confident \\
Very uncomfortable about being & Not at all uncomfortable about \\
aggressive & being aggressive \\
Not at all ambitious & Very ambitious \\
Unable to separate feelings from & Easily able to separate feelings from \\
ideas & ideas \\
Very dependen & Not at all dependent \\
Very conceited about appearance & Never conceited about appearance \\
Thinks women are always superior & Thinks men are always superior to \\
to men & men \\
Does not talk freely about sex with & Talk freely about sex with men \\
men & \\
\hline & \\
\hline
\end{tabular}


Table 1

Continued

b) Feminine pole is more desirable

\begin{tabular}{ll}
\hline$\underline{\text { Feminine }}$ & $\underline{\text { Masculine }}$ \\
\hline Does not use harsh language at all & Uses very harsh language \\
Very talkative & Not at all talkative \\
Very tactful & Very blunt \\
Very gentle & Very rough \\
Very aware of feeling of others & Not at all aware of feeling of others \\
Very religious & Not at all religious \\
Very interested in own appearance & Not at all interested in own appearance \\
Very neat in habits & Very sloppy in habits \\
Very quiet & Very loud \\
Very strong need for security & Very little need for security \\
Enjoys art \& literature & Does not enjoy art \& literature at all \\
Easily expresses tender feelings & Does not express tender feelings at all \\
\hline
\end{tabular}

Source: Broverman et al., 1972 cited in Nicholson, 1984, p. 8.

The traits on women particularly describe how women in a given society behave and prescribe how they should behave (Haslett \& Lipman, 1997 cited in Gillem, Sehgal, \& Forcet, 2000, p. 55). The social norms regarding expected behaviours of women push them into a double jeopardy. Women are blamed having not feminine qualities if their conduct is found similar with those specified for men, and if they adopt prescribed behaviour that is meant to be feminine, they are inadequate in comparison with the behaviour prescribed for men (Gillem et al., 2000, p. 55).

In the 1970's, Sandra Bem has identified some characteristics, which are considered desirable according to a person's sex. Interestingly, most men and women tend to choose and describe characteristics or traits, which are either masculine or feminine for them. However, individuals also find themselves as possessing both types of characteristics or traits equally depending on the situational appropriateness. People who have both types of characters might be androgynous (Bem, 1974, p. 155). Following this idea, Sandra Bem, has developed a new kind of inventory scale-the Bem Sex-Role Inventory (BSRI) that is founded on a conception of the sex-typed 
person of someone who has internalised society's desirable standard of being masculine and feminine and also a list of neutral attributes equally desirable in a male or a female (Bem, 1974, p. 156).

Table 2

Items on Masculinity, Femininity and Social Desirability Scale of the BSRI

\begin{tabular}{|c|c|c|}
\hline Masculine items & Feminine items & Natural items \\
\hline 49 Acts as leader & 11 Affectionate & 51 Adaptable \\
\hline 46 Aggressive & 6 Cheerful & 36 Conceited \\
\hline 58 Ambitious & 50 Childlike & 9 Conscientious \\
\hline 22 Analytical & 32 Compassionate & 60 Conventional \\
\hline 13 Assertive & 53 Does not use harsh language & 45 Friendly \\
\hline 10 Athletic & 35 Eager to soothe hurt feeling & 15 Нарpy \\
\hline 55 Competitive & 20 Feminine & 3 Helpful \\
\hline 4 Depends on own belief & 14 Flatterable & 48 Inefficient \\
\hline 37 Dominant & 59 Gentle & 24 Jealous \\
\hline 19 Forceful & 47 Gullible & 39 Likable \\
\hline 25 Has leadership abilities & 56 Loves children & 6 Moody \\
\hline 7 Independent & 17 Loyal & 21 Reliable \\
\hline 52 Individualistic & 26 Sensitive to the needs of others & 30 Secretive \\
\hline 31 Makes decisions easily & 8 Shy & 33 Sincere \\
\hline 40 Masculine & 38 Soft spoken & 42 Solemn \\
\hline 1 Self-reliant & 23 Sympathetic & 57 Tactful \\
\hline 34 Self-sufficient & 44 Tender & 12 Theatrical \\
\hline 16 Strong personality & 29 Understanding & 27 Truthful \\
\hline 43 Willing to take a stand & 41 Warm & 18 Unpredictable \\
\hline 28 Willing to take risks & 2 Yielding & 54 Unsystematic \\
\hline
\end{tabular}

Note: The number preceding each item reflects the position of each adjective as it appears in the inventory.

Source: Bem, 1974, p. 156.

Twenty items reflect the definition of masculinity; twenty reflect the definition of femininity and twenty are the fillers. Bem's instrument was the first attempt to specifically provide independent measures of an individual's masculinity and femininity (Lenney, 1991), and it has produced relatively enduring definitions of masculinity and femininity. 
Bem's findings are being used widely in empirical studies on the nature of masculinity and femininity (Choi \& Fuqua, 2003, p. 872).

Though sex role theory is an essential one to help to differentiate traits between masculinity and femininity, it is not free from criticism. It was criticised by the feminists for its reliance on biological determinism and its incapability of conceptualisation of gender relations existing in social institutions and reproduced in interpersonal interactions (Smith, 1987, and West \& Zimmerman, 1987 cited in Allen, Lloyd, \& Few, 2009, p. 8). According to Connell (1987, p. 53-54), sex role theory is abstract in nature as well as it is unable to reflect a complete picture of the differences and relationships between men and women since it lacks a distinct method to theorise gender historically and socially. It lacks in the theorisation of power, social interests or social dynamism. Moreover, in sex role theory, roles are viewed as static and unchanging, and these are more prescriptive (Prentice, 2000, p. 223). Yet, the fact is that roles of an individual are always changeable, and an individual can easily switch his roles in response to various needs and demands. Moreover, the existing assumption of a normative standard of masculinity is seemingly improper and inadequate. The approach, therefore, has been overtaken by other theoretical approaches of masculinity (Pease, 2007, p. 555). Even though it is a very influential theory which identifies or separates the notions and norms regarding masculinity and femininity in gender relations, and in turn, maintains stout relationships in the perpetuation of violence against women.

\section{Theory of Hegemonic Masculinity}

Raewyn Connell, an Australian sociologist, is the most influential contemporary theorist of masculinity study and research. In her work, 'Gender and power: Society, the person and sexual politics' (1987), the concept of hegemonic masculinity was constructed. The idea of different forms of masculinities was central as well as crucial to an understanding of how patriarchal social order works (Connell, 1987, p. 183). Connell argues that there are different forms of masculinities, but one form is culturally esteemed above all other. Connell has termed that as hegemonic masculinity or dominant form of masculinity. By using this term, she has developed a process of stratifying masculinities (McCormack, 2012, p. 37). 
Specifically speaking, Connell rejects the conceptual singularity of masculinity and views masculinity as masculinities. Her hegemonic masculinity is largely symbolic, legitimate and ideal type of masculinity within the multiplicity of masculinities. This typical and lawful form of masculinity emerges and develops within a particular socio-cultural milieu and acts as a benchmark, which all men must follow in maintaining their gender order (Howson, 2006, p. 3). However, it is also true that hegemonic masculinity is not something, which is fixed or constant. It is a masculinity that occupies the hegemonic position in a given society and pattern of gender relations, and it is always contestable (Connell, 1995, p. 76). It is to note that Connell in some of her influential texts $(1987 ; 1995 ; 2000)$ has developed and devised a broad and interdisciplinary theoretical framework for analysing masculinity (Ashe, 2007, p. 143).

Connell selected the idea of hegemony from Antonio Gramsci's (1978) analysis of class relations in Italy. Gramsci's analysis is based on the cultural dynamics by which a group claims and sustains a leading position in social life (Connell, 1995, p. 77). According to Gramsci, this position is primarily obtained by consent rather than force and also suggests that force may be used if a group does not give permission either actively or passively (Messerschmidt, 1993, p. 82). Hegemony, in fact, refers to a form of dominance, which legitimates and sustains the position of the ruling class over the ruled (Wong, 2000, p. 241). Hegemonic masculinity is defined as a gendered practice that provides explanations of the legitimacy of patriarchy and ensures the dominant position of men and the subordination of women (Connell, 1995, p. 77). It is, thus, a vital tool for feminist analysis of patriarchy since it specifically reflects gender issue (Wong, 2000, p. 241). Connell further argues that the gender dynamics of the idea of hegemony is employed to explain how certain ideals of masculinity justify the unequal relations between men and women in a patriarchal culture (Ashe, 2007, p. 146).

Hegemonic masculinity is indubitably the normative pattern of masculinity and nurturing of this kind of masculinity is the most dignified way of being a man (Connell \& Messerschmidt, 2005, p. 832). Connell (1995, p. 78) has identified some important features of hegemonic masculinity such as i) hegemony relates to cultural dominance in the society. It represents the dominance of heterosexual men and subordination 
of homosexual men, and ii) the majority of men gain from hegemonic masculinity as they benefit from the patriarchal dividend. The patriarchal dividend is regarded as a form of gender practice that entails taking general advantage from the overall subordination of women. Notably, other authors have also used the term hegemonic masculinity. Kimmel (1994, p. 125) opines that hegemonic masculinity refers to a man in power, a man with power and a man of power. The definition of this type of masculinity lies in culture, and that culture promotes of maintaining the power of some men over other men and men over women. Lorber (1998 cited in Flood, 2002, p. 207) sates men who are economically prosperous, racially superior and apparently heterosexual are the true representatives of hegemonic masculinity. Donaldson (1993 cited in Howson, 2006, p. 3) has defined hegemonic masculinity as culturally idealised form of masculinity. It is centrally connected with the institutions of male dominance, not all men practice it, though most men take benefit from it. It is pseudo-natural, tough, contradictory, crisis-prone, wealthy and socially sustained and it often excludes working-class, gay and black-men. Hegemonic masculinity apparently highlights and promotes the practices of authority, control, competitive individualism, independence, aggressiveness, and develops the capacity for condoning violence (Connell, 1990, 1992, and, Segal, 1990 cited in Messerschmidt, 1993, p. 82). Therefore, men who do not conform to the hegemonic definition of masculinity are treated to be feminine (Yeung, Stombler, \& Wharton, 2010, p. 149). Hegemonic masculinity fits the contemporary needs of the social and gender order (Duffy \& Momirov, 1997, p. 124), and as it is the idealised form of masculinity as it is culturally honoured, glorified and sustained (Connell, 1990, 1992 cited in Messerschmidt, 1993, p. 82).

Beginning from the mid-1980s, the concept of hegemonic masculinity has been widely used in various ways, and it has also been an issue of debate and criticism (Connell \& Messerschmidt, 2005, p. 832). Mike Donaldson (1993 cited in Hearn, 2004, p. 58) has marked that the concept of hegemony is unclear and contradictory, often vague and ambiguous as it is unable to answer the pertinent questions regarding issues and practices of hegemony (Hearn, 2004, p. 58). The concept is viewed as 'over simplified' since it lacks in producing a subtle relationship between sex and gender. The idea of hegemonic masculinity has particularly come to be negatively 
associated with men, and it refuses to recognise anything positive with men (Collier, 1998 cited in Dowd, 2010, p. 53). It also assumes that as men have the power, they cannot have virtue on their side (Seidler, 200, p. 52). Hegemonic masculine ideals and practices play a role in maintaining the current gender order and in so doing it generates barriers to gender equality (Davis, 1997, p. 84).

Even in the facet of criticism, the concept hegemonic masculinity provides rich resources for analysis of issues related to subordination and domination of women including violence against women through the lens of feminist theory and practice. Galdas (2009, p. 70) regards hegemonic masculinity as the most honoured, desired and culturally authoritative form of masculinity in particular given context. It stands as the standard for all other men to follow and also against which other men are measured. Koeing (2007, p. 28) states men who have control and power perform hegemonic masculinity and men who lack power and control perform complicit or subordinated masculinity. Consequently, a hegemonic form of masculinity is the superior form of masculinity.

\section{Revisiting Theories \& Interconnecting with Violence against Women}

The theoretical and conceptual discussions above provide indications to highlight how masculinity is linked to violence against women. Recognising masculinity or maleness as a resource, feminist researchers have argued that men use this resource through perpetuating violence against women to reproduce and maintain their relative status and authority over women (Adler, 1997, p. 436). Alternatively, various forms of violence against women are the manifestations of a dynamic form of masculinity that separate men's activity from femininity (Kaufman, 1997, p. 41). In short, masculinity is what is made, and violence against women is a resource of making masculinity (Crosset, 2000, p. 155). However, both Connell (1995, p. 83) and Messerschmidt (1993, p. 45) have suggested not to assume that all men use violence to maintain authority over women. It is also to note that besides presuming violence there are other means of showing masculinity (Connell, 2000 cited in DeKeseredy \& Schwartz, 2005, p. 356). Freud's psychoanalytical theory is significantly relevant to violence against women. The male phallus is the tool and the primary source of power in 
heterosexual relationship towards subordination of and control over women. The actual use of the phallus is to threaten women (Stanly, 2000, p. 1535). A man takes pride in his phallus, and this pride demonstrates his assertiveness, aggression and strength through the means of speaking, extending, thrusting, and penetrating. More specifically, the phallus is characterised by self-display, haughty reserve, and an instrument of aggression, recklessness, misogyny, and an excessive need to display one's potency (Diamond, 2006, p. 1101). Haddon (1998 cited in Cossins: 2000, p. 119) states that the key characteristics of masculine sexual ideals are; potent, penetrating, outward thrusting, initiating, forging ahead into virgin territory, opening the way, sword line, able to cut through, able to clear or differentiate, goal-oriented, to the point, focused, directive, effective, aimed, hitting the mark, strong, erect. As a matter of fact, a man uses this powerful tool in a heterosexual relationship to show his manhood in many forms of violence against women such as marital rape, sex without wife's consent, sex as and when he desires, impregnating wife against her desire and obnoxious sexual behaviours. Sexual performance and virility are central to the construction of masculine sexual performances. Conversely, impotence is a matter of shame for men and a great thereat to masculinity. In such circumstances heterosexual men often engage in different types of violence to overcome feelings of shame and dishonour (Higgins, 2006, p. 88). Many forms of violence are perpetuated in heterosexual relations to demonstrate or retain masculinity. For example; Messerschmidt (1993, p. 151) states that marital rape is a specific type of patriarchal masculinity in heterosexual relations targeted towards domination and control of women's sexuality. Hong (2000, p. 272) states that the norm of heterosexuality is to have sex more often with a female partner and this is how the masculinity is also ensured. On the other hand, wife-beating is common not only for her failure of performing essential obligations rightly but also for her challenge to his dominance and control. In such a situation wife-beating is an important tool through which a man tries to re-establish his masculinity (Messerschmidt, 1993, p. 145 \& 148). Wenbeirg (1982, p. 16) also comments that the motivation for all male violence is related to attempts of men to reinforce their heterosexual masculinity.

The relation between sex role theory and violence against women is very strong. Many of the traits or constructs developed in different orientations 
of sex role theory have direct linkages with acts of violence against women. The sex role theory was theorised to reveal the source of gender oppression and men's domination over women. It has significant implications for feminist theory since it has put particular emphasis on the realities of structural inequalities between men and women (Newton, 2002, p. 185), and on socialising men to be the oppressor and to use force to maintain domination if deemed necessary (Brownridge \& Halli, 2001, p. 33). Feminist sociologists point out that the prevailing notion of masculinity and femininity as developed by sex role theory tends not to separate males and females but also infuses into them the framework of conflict and violence (Mackie, 1991 cited in Duffy \& Momirov, 1997, p. 133). The socialisation process of assigning sex role behaviour (masculine or feminine) contributes greatly to violence against women. Women survivors of violence are socialised more rigidly to female sex roles (feminine) stereotypes, and violent men are more rigidly socialised to male sex roles (masculine) expectations (Walker, 2000, p. x). Socialisation prepares women to be the legitimate victims and men to be the potential offenders (Weis \& Borges, 1973 cited in Scully \& Marolla, 2005, p. 17). When these roles are challenged, a crisis in masculine identity may emerge, and a man may use violence against women in response to the perceived loss of, and attack on his masculine identity (Shefer \& Ruiters, 1998, and, Simpson, 1992 cited in Boonzaier \& Rey, 2003, p. 1020). Hence, every construct developed from sex role theory provides fundamental meanings of masculinity as well as agreeably internment flexible interface with violence against women.

Hegemonic masculinity stands as a compelling ideology that provides justifications that patriarchy is legitimised and must be maintained (Levy, 2007, p. 254). In feminist theory, women's subordination under patriarchal structure is viewed as the cause of violence against women. Therefore, the relation between hegemonic masculinity and violence against women is very close. The practice of hegemonic masculinity attempts to control women's subjectivity so that women provide sexual, practical, maternal and emotional services to men (Hird, 2002, p. 83). Men enjoy the material, physical and symbolic benefits of the subordination of women by practising hegemonic masculinity-the 'ideal-type' masculinity (Levy, 2007, p. 254). In patriarchal culture, men enjoy full control over women because patriarchy gives them the power and authority to do so. Connell (1995, p. 
83) views that men use violence against women to sustain and retain their dominance, control and power. In addition to that, men who condone violence are unlikely to view them as deviant acts which are entirely justified; they are exercising a right which stems from the authority by an ideology of supremacy or hegemony. Therefore, the connection between hegemonic masculinity and violence is very intimate.

It is also important to note that the perceived crisis in masculinity also leads to domestic violence against women. Crisis in masculinity is something which is disrupted or in the process of transformation within a system of gender relations (Connell, 1995, p. 85). Kimmel (2003 cited in Ikeda, 2007, p. 116) opines the displacement of men from their traditional roles, responsibilities and power is viewed as the crisis in masculinity. As a matter of fact, deviation from the prescriptions given in each of the theories reflects the crisis in masculinity. The frustration from such a displacement or crisis might lead to the propagation of many forms of violence. Intensifying poverty and inequality, increasing unemployment, changing livelihood system, empowerment of women and greater control over resources by women and ideology have contributed to the crisis of normative masculinity (Batliwala, 1994 cited in Amuyunzu-Nyamongo \& Francis, 2006, p. 219). Over the years, men have been facing a gradual erosion of both actual male power and symbolic power of the traditional concept of masculinity (Pfeil, 1995 cited in Robinson: 1997, p. 90). It is evident that women have substantially been empowered and taken up more instrumental roles for household activities which act as contributing factor towards pushing men's masculinity in crisis. In that case, men may strive to reassert their masculinity turning to violence means (Amuyunzu-Nyamongo \& Francis, 2006, p. 220). Lindorfer, (2007, p. 32) also opines that due to macro social changes men at times may lose their position as the sole breadwinner, their status and role in the household and community may be undermined. Men, therefore, take violence against women as resort to reinstate their masculinity, on the other hand, traditional male characteristics, roles and responsibilities are also perceived to be linked to social problems and are not viewed as positive characteristics of manhood or masculinity (Gilbert, 1992, and, Levant, 1997 cited in Kahn, 2009, p. 198). MacInees (2001 cited in Kahn, 2009, p. 198) also views that traits like courageous, heroism, independent, rational, virility, strong etc. were once 
claimed as masculine virtues; but these have turned into masculine vices like destructive, aggression, abusive and detachment. Apparently, this is a tense situation for normative masculinity, as such, a crisis in masculinity. If women take advantage over gender relations or doubt about men's perceived superiority and act against men's dominant role, it is very likely that men will consider their masculinity to be in a state of crisis. In such a crisis men will make attempts to restore or reinstate their masculinity (Connell, 1995, p. 84). No doubt, violence against women is the most powerful tool for men to restore their destroyed masculinity as the crisis in masculinity brings the risk of losing everything for them (Yekani, 2011, p. 25).

\section{Conclusion}

In this paper, attempts have been made to focus on the whole gamut of masculinity and its linkage to violence against women. As the constructs of masculinity vary over time, space and culture, it is challenging to have a unified standard of masculinity. Nonetheless, it is a very relevant analytical tool of gender relations. Masculinity is exactly the opposite notion of femininity. The analysis of masculinity is only perfect when it is compared to femininity. Though the concept masculinity has some meanings and has the certain set of traits but its dominant theoretical sources such as psychoanalysis, sex-role and hegemony provide abundant elements of analysing the concept. Psychoanalytical theory gives a biological framework for showing one's masculinity; sex role theory socialises on how to internalise or practice masculinity and theory of hegemony provides the basis on how to become the perfect and powerful man. The theoretical notions of masculinity further confirm that they are closely associated with the practice of violence against women. Violence is an effective means to dominate women. Men practice violence not only to demonstrate his masculinity but also to safeguard it. If a man feels that his masculinity is in danger or crisis, he may also be violent to reinstate that. Nonetheless, masculinity enables and encourages men to dominate and control women. To become masculine, at times, a man might take up the role of oppressor. Perpetuating violence against women is a demonstration of masculinity in gender relations. 
Being a review research, it has certain limitations, which can be compensated by empirical research. It would be interesting to see a country specific broader survey of masculinity, which might include understanding of the construction of masculinity and the implications of masculinity theories in understanding violence against women. Finally, it is strongly suggested that masculinity is a concept that demands systematic and categorical understanding particularly about violence against women so that effective measures can be taken up to bring changes amongst men, and to establish a balanced and mutually respectful society.

\section{References}

Abbott, P. (2000). Masculinity. In C. Kramerae \& D. Spender (Ed.), International encyclopaedia of women (pp. 1312-1313). New York: Routledge.

Adams, M., \& Coltrane, S. (2005). Boys and men in families. In M.S. Kimmel, J. Hearn \& R. Connell (Eds.). Handbook on studies on men and masculinities (pp. 230-248). Thousand Oaks: Sage Publications,. Adler, C. (1997). Violence, gender and social change. In L. L. O’Toole \& J. R. Schiffman (Eds.), Gender violence: interdisciplinary perspectives (pp. 435-442). New York: New York University Press.

Ahmed, D. S. (2006). Gender and Islamic spirituality: A Psychological view of 'low' fundamentalism, In L. Ouzgane (Ed.), Islamic masculinities (pp. 11-34). London: Zed Books.

Allen, K. R., Lloyd, S. A., \& Few, A. L. (2009). Reclaiming feminist theory, method and praxis for family studies. In S. A. LIoyd, A. L. Few \& K. R. Allen (Eds.), Handbook of feminist family studies (pp. 3-19). Thousand Oaks: Sage Publications.

Alvesson, M., \& Billing, Y. D. (1997). Understanding gender and organisations. London: Sage publications.

Amuyunzu-Nyamongo, M., \& Francis, P. (2006). Collapsing livelihoods and crisis of masculinity in rural Kenya. In I. Bannon \& M. C. Corriea (Eds.), The other half of gender (pp. 219-244). Washington DC: The World Bank.

Ashe, F. (2007). The new politics of masculinity: Men, power and resistance. Oxon: Routledge. 
Beere, C. A. (1990). Gender roles: A handbook of tastes and measures. Connecticut: Greenwood Publishing.

Bem, S. (1974). The measurement of psychological androgyny. Journal of Consulting and Clinical Psychology, 42(2), 155-162. Retrieved from https://www.ncbi.nlm.nih.gov/pubmed/4823550

Bem, S. (1993). The lenses of gender: Transforming the debate on sexual inequality. New Haven: Yale University Press.

Blanchet, T., Biswas, H., \& Lucky, M. A. (2001). Constructions of masculinities and violence against women. Dhaka: CARE.

Boonzaier, F., \& Rey, C. d. L. (2003). He is a man, and I'm a woman:

Cultural constructions of masculinity and femininity in South African women's narratives of violence. Violence against Women, 9(8), 1003-1029. doi:10.1177/1077801203255133

Brannon, R. (1976). The male sex role: Our culture's blueprint for manhood and what it's done for us lately. In D. David \& R. Brannon (Eds.), The forty-nine percent majority: The male sex role (pp. 1-49). Reading, MA: Addison-Wesley.

Brownridge, D. A., \& Halli, S. S. (2001). Explaining violence against women in Canada. Lanham: Lexington Books.

Connell, R.W. (1987). Gender and power: Society, the person and sexual politics. Stanford CA: Stanford University Press.

Connell, R.W. (1994). Psychoanalysis on masculinity. In H. Brod \& M. Kaufman (Eds.), Theorising masculinities (pp. 11-38). London: Sage Publications.

Connell, R.W. (1995). Masculinities. Berkley: University of California Press.

Connell, R.W. (2000). Relations among masculinities. In M. Kimmel \& A. Aronson (Eds.), Men and masculinities: A social, cultural and historical encyclopaedia (pp. 508-510). Santa Barbara, CA: ABCCLIO.

Connell, R.W. (2001). The social organisation of masculinity. In S. M. Whitehead \& Frank J. Barrett (Eds.), The masculinities reader (pp. 30-50). Cambridge: Polity.

Connell, R.W. (2002). Gender. Cambridge: Polity Press. 
Connell, R.W, \& Messerschmidit, J.W. (2005). Hegemonic masculinities:

Rethinking the concept. Gender and Society, 19(6), 829-859.

doi:10.1177/0891243205278639

Cornwall, A., \& Lindisfarne, N. (1994). Dislocating masculinity: Gender,

power and anthropology. In A. Cornwall \& N. Lindisfarne (Eds.),

Dislocating masculinity: Comparative ethnographies (pp.11-47).

London: Routledge.

Cossins, A. (2000). Masculinities, sexualities and child sexual abuse. The Hague: Kluwer Law International.

Choi, N., \& Fuqua, D. R. (2003). The structure of BEM sex role inventory:

A summery report of 23 validation studies. Educational and

Psychological Measurement, 63(5), 872-887.

doi:10.1177/0013164403258235

Crosset, T. (2000). Athletic affiliation and violence against women: Toward a structural prevention project. In J. McKay, M. A. Messner \& D. F. Sabo (Eds.), Masculinities, gender relations and sports (pp. 147161). Thousand Oaks: Sage Publications.

Davis, L. R. (1997). The swimsuit issue and sport: Hegemonic masculinity in sports illustrated. New York: State University of New York Press.

DeKeseredy, W. S., \& Schwartz, M. D. (2005). Masculinities and interpersonal violence. In M. S. Kimmel, J. Hearn \& R. W. Connell (Eds.), Handbook of studies on men and masculinities (pp. 353-366). London: Sage Publications.

Dowd, N. E. (2010). The man question: Male subordination and privilege. New York: New York University Press.

Diamond, M. J. (2006). Masculinity unravelled: The roots of male gender identity and the shifting of male ego ideals throughout life. Journal of the American Psychoanalytical Association, 54(4), 1099-1130. doi:10.1177/00030651060540040601

Duffy, A., \& Momirov, J. (1997). Family violence: A Canadian introduction. Toronto: Lorimer.

Edwards, T. (2006). Culture of masculinity. London: Routledge.

Ferrell, R. (2000). Psychoanalysis. In L. Code (Ed.), Encyclopaedia of feminist theories (pp. 403-406). London: Routledge.

Fischer, A. R., \& Good, G. E. (1998). New directions for the study of gender role attitudes: A cluster analytical investigation of masculine 
identities. Psychology of Women Quarterly, 22(3), 371-384. doi:10.1111/j.1471-6402.1998.tb00163.x

Flanagan Jr, O. J. (1982). Freud: Masculinity, femininity and the philosophy of the mind. In M. Vetterling-Braggin (Ed.), "Femininity", "masculinity" and "androgyny": A modern philosophical discussion (pp. 60-76). Totowa: N.J: Rowman and Littefield Publication Inc.

Flood, M. (2002). Between men and masculinity: An assessment of the term "masculinity" in research scholarship on men. In S. Pearce \& V. Muller (Eds.), Manning the next Millennium: studies in masculinities (pp. 203-213). Bentley: Black Swan Press.

Friedan, B. (1963). The feminist mystique. Harmondsworth: Penguin Books.

Friedman, R. C., \& Downey, J. I. (2002). Sexual orientation and psychodynamics psychotherapy: Sexual science and clinical practice. New York: Colombia University Press.

Galdas, P. M. (2009). Men, masculinities and help-seeking behaviour. In A. Broom \& P. Tovey (Eds.) Men's health, body, identity and social context (pp. 63-77). Chichester: Willey-Blackwell.

Gardiner, J. K. (2002). Feminism and psychoanalysis. In E. Erwin (Ed.), The Freud encyclopaedia: Theory, therapy and culture (pp. 195199). Routledge: New York,.

Giddens, A. (2001). Sociology. Cambridge: Polity.

Gillem, A. R., Sehgal, R., \& Forcet, S. (2000). Understanding prejudice and discrimination. In M. Biaggio and M. Hersen (Eds.), Issues in the psychology of women (pp. 55-70). New York: Plenum Publishers.

Good, G. E., Borst, T. S., \& Wallace, D. L. (1994). Masculinity research: A review and critique. Applied and Preventive Psychology, 3(1), 3-14. doi:10.1016/S0962-1849(05)80104-0

Gramsci, A. (1978). Selections from political writings 1921-1926. New York: International Publishers.

Harris, I. M. (1995). Messages men hear: Constructing masculinities. Bristol: Taylor and Francis.

Hearn, J. (1998). The violences of men: How men talk about and how agencies respond to men's violence to women. London, UK: SAGE.

Hearn, J. (2004). From hegemonic masculinity to the hegemony of men. Feminist Theory, 5(1), 49-72. doi:10.1177/1464700104040813 
Hearn, J. (2007). Masculinity/Masculinities. In M. Flood, J. K. Gardiner, B. Pease \& K. Pringle (Eds.), International encyclopaedia of men and masculinities (pp. 390-394). London: Routledge.

Higgins, D. (2006). Narcissism, the adonis complex, and the pursuit of the ideal. In C. Kendall \& W. Martino (Eds.), Gendered outcastes and sexual outlaws (pp. 79-100). Binghamton, NY: The Haworth Press Inc.

Hird, M. J. (2002). Endangering violence. Ashgate: Aldershot.

Hoffman, R. M., Hattie, J. A., \& Borders, L. D. (2005). Personal definitions of masculinity \& femininity as an aspect of gender self-concept. Journal of Humanistic Counselling, Education and Development, 44(1), 66-83. doi:10.1002/j.2164-490X.2005.tb00057.x

Hong, L. (2000). Toward a transformed approach of prevention: Breaking the link between masculinity and violence. Journal of American College Health, 48(6), 269-279. doi:10.1080/07448480009596268 Horowitz, G., \& Kaufman, M. (1987). Male sexuality: Toward a theory of liberation. In M. Kaufman (Ed.), Beyond patriarchy: Essays by men on pleasure, power and change (pp. 81-102). Toronto: Oxford University Press.

Howson, R. (2006). Challenging hegemonic masculinity. Oxon: Routledge. Ikeda, S. (2007). Masculinity and masculinism under globalisation: Reflections on Canadian case. In M. G. Cohen \& J. Brodie (Eds.), Remapping gender in new global order (pp.111-130). Abingdon: Routledge.

Kahn, J. S. (2009). An introduction to masculinities. Chichester: WileyBlackwell.

Kaufman, M. (1987). The construction of masculinity and the triad of men's violence. In M. Kaufman (Ed.), Beyond patriarchy: Essays by men on pleasure, power and change (pp. 1-29). Toronto: Oxford University Press.

Kaufman, M. (1997). The construction of masculinity and the triad of men's violence. In L. L O'Toole \& J. R. Schiffman (Eds.), Gender violence: Interdisciplinary perspectives (pp. 30-51). New York University Press: New York. 
Kilmartin, C. (2004). Freud, Sigmund. In M. Kimmel \& A. Aronson (Eds.), Men and masculinities-A social, cultural \& historical encyclopaedia (pp, 319-321). Santa Barbara, CA: ABC-CLOI, Inc.

Kimmel, M. S. (1994). Masculinity as homophobia-fear, shame and silence in the construction of gender identity. In H. Brod \& M. Kaufman (Eds.), Theorising masculinities (pp. 119-141). Thousand Oaks: Sage Publications.

Koenig, N. K. (2007). An analysis of the construction of masculinity in the "says her" feature of maxim magazine (Masters dissertation). The University of Louisville, Kentucky.

Lafrance, M. R. (2004). Psychoanalysis. In M. Kimmel \& A. Aronson (Eds.), Men and masculinities: A social, cultural and historical encyclopaedia (pp. 642-644), Santa Barbara CA: ABC-CLIO Inc.

Lenney, E. (1991). Sex roles: The measurement of masculinity, femininity, and androgyny. In J. P. Robinson, P. R. Shaver \& L. S. Wrightsman (Eds.), Measures of personality and social psychological attitudes (pp. 573-660). San Diego, CA: Academic Press.

Levy, B. (2008) Women and violence. Berkeley, Seal Press.

Lindorfer, S. (2007). Sharing the pains of the bitter hearts. London: Transaction Publishers.

Mackie, M. (1980). The impact of sex stereotypes upon adult self imagery. Social Psychology Quarterly, 43(1), 121-145. Retrieved from http://www.jstor.org/stable/3033755

Mahalik, J. R. (2000). Gender role strain. In M. Kimmel \& A. Aronson (Eds.), Men and masculinities: A social, cultural and historical encyclopaedia (pp. 350-351). Santa Barbara, CA: ABC-CLIO.

Martin, H., \& Finn, S. E. (2010). Masculinity and femininity. Minneapolis: University of Minnesota Press.

Mason-Grant (2004). Masculinity. In L. Code (Ed.), Encyclopaedia of feminist theories (pp. 322-323). London: Routledge.

McCormark, M. (2012). The declining significance of homophobia: How teenage boys are redefining masculinity and heterosexuality. Oxford: Oxford University Press.

Messerschmidt, J. W. (1993). Masculinities and crime: Critique and reconceptualisation of theory. Maryland: Rowman and Littlefield Publishers. 
Newton, J. (2002). Masculinity studies: The longed for profeminist movement for academic men. In J. K. Gardiner (Ed.), Masculinity studies and feminist theory (pp. 161-175). New York: Columbia University Press.

Nicholson, J. (1980). Men and women: how different are they? New York: Oxford University Press.

Parsons, T., \& Bales, R. F. (1956). Family, socialisation and interaction process. London: Routledge.

Pease, B. (2007). Sex role theory. In M. Flood, J. K. Gardiner, B. Pease, and K. Pringle (Eds.) International encyclopaedia of men and masculinities (pp. 554-555). London: Routledge,

Person, E. S. (2009). Masculinities, plural. In B. Reies \& R. Grossmark (Eds.), Heterosexual masculinities: Contemporary perspectives (pp. 1-21). New York: Routledge.

Prentice, S. L. (2000). Gender roles. In L. Code (Ed.) Encyclopaedia of feminist theories (pp, 222-223). London: Routledge.

Robinson, S. (2007). Crisis in masculinity. In M. Flood, J. K. Gardiner, B. Pease, \& K. Pringle (Eds.), International encyclopaedia on men and masculinities (pp. 90-91). London: Routledge.

Sahoo, F. M. (2004). Sex roles in transition. Delhi: Kalpaz Publications. Scully, D., \& Marolla, J. (2005). Riding the bull at gilley's: Convicted rapists describe the rewards of rape. In C. M. Renzendtti \& R. K. Bergen (Eds.), Violence against women (pp. 15-30). Lanham: Rowman and Littlefield Publishers.

Seidler, V. J. (2006). Transforming masculinities. Oxon: Routledge.

Serafica, F. C., \& Rose, S. (1982). Parents' sex role attitudes and children's concept of femininity and masculinity. In J. Downing (Ed.), Sex role attitude and cultural change (pp. 11-24). Hingham, M A: D. Reidel Publishing Company.

Stanly, L. (2000). Phallocentrism. In C. Kramerae \& D. Spender (Eds.), International encyclopaedia of women (pp. 1535.). New York: Routledge.

Thomas, C. (2007). Psychoanalysis. In M. Flood, J. K. Gardiner, B. Pease $\&$ K. Pringle (Eds.), International encyclopaedia on men and masculinities (pp. 512-515). London: Routledge.

Turner, P. (1995). Sex, gender and identity. Leicester: Wiley-Blackwell. 
Walczak, Y. (1988). He and she: Men in eighties. London: Routledge.

Walker, L. E. A. (2000). The battered woman syndrome. New York:

Springer.

Wenbeirg, J. (1982). How homophobia affects all boys and men. Teaching Sexual Ethics Newsletter, 2, 10-17.

Whitehead, S. M. (2002). Men and masculinities-Key themes and new directions. Cambridge: Polity Press.

Whitford, M. (2000). Psychoanalysis. In C. Kramarae \& D. Speder (Eds.), International encyclopaedia of women (pp. 1684-1687). New York: Routledge.

Wong, Y. (2004). Hegemony. In L. Code (Ed.), Encyclopaedia of feminist theories (pp. 241-242). London: Routledge.

Yekani, E. H. (2011). The privilege of crisis. Frankfruit: Campus.

Yeung, King-T., Stombler, M., \& Wharton, R. (2010). Making men in gay fraternities: Resting and reproducing multiple dimensions of hegemonic masculinity. In S. R. Harper \& F. Hariss III (Eds.), College men and masculinities (pp., 148-171). San Francisco: John Willy and Sons.

Anisur Rahman Khan, Assistant Professor \& Chairperson, Department of Sociology, East West University, Dhaka, Bangladesh.

Shahriar Khandaker, Senior Lecturer, Department of Social Relations, East West University, Dhaka, Bangladesh.

Contact Address: Direct correspondence to Anisur Rahman Khan, Department of Sociology, East West University, A/2, Jahurul Islam Avenue Jahurul Islam City, Aftabnagar Dhaka-1212, Bangladesh, email:arkhan@ewubd.edu 


\section{Hipatia Press \\ www.hipatiapress.com}

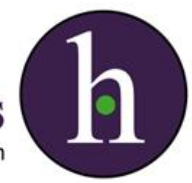

Instructions for authors, subscriptions and further details:

http://mcs.hipatiapress.com

\section{Masculinity and Rhythmic Gymnastics. An Exploration on the Transgression of Gender Order in Sport}

Joaquín Piedra ${ }^{1}$

1) University of Sevilla, Spain

Date of publication: October $21^{\text {st }}, 2017$

Edition period: February 2018 - June 2018

To cite this article: Piedra, J. (2017). Masculinity and Rhythmic

Gymnastics. An Exploration on the Transgression of Gender Order in Sport. Masculinities and Social Change,6(3), 288-303. doi:

10.17583/MCS.2017.2733

To link this article: http://doi.org/10.17583/MCS.2017.2733

PLEASE SCROLL DOWN FOR ARTICLE

The terms and conditions of use are related to the Open Journal System and to Creative Commons Attribution License (CC-BY). 
MCS - Masculinities and Social Change Vol. 6 No. 3 October 2017 pp. 288-303

\section{Masculinity and Rhythmic \\ Gymnastics. An Exploration on the Transgression of Gender Order in Sport}

Joaquín Piedra

University of Sevilla

\section{Abstract}

Patriarchal dominance in our Western society has oppressed not only women but it has also isolated many men who did not comply with hegemonic masculinity patterns. The aim of this study is to know and analyse the experiences of a group of boys who practise rhythmic gymnastics, traditionally linked to the feminine reality. Within this interpretative paradigm, eight interviews have been done to gymnasts (819 years old), who practise rhythmic gymnastics in a competitive or amateur level. The boys express the familiar support that they have had when deciding to practise a traditionally feminine sport. However, many gymnasts have been insulted or mocked by other boys. Therefore, it is important to work with families in order to eliminate stereotypes and prejudices in this masculine discipline in the future. Similarly, rhythmic gymnastics must be promoted among boys, since a higher presence of boys in clubs would ease their reception among girls.

Keywords: masculinity, gender identity, gymnastics, youth. 
MCS - Masculinities and Social Change Vol. 6 No. 3 October 2017 pp. 288-303

\section{Masculinidad y Gimnasia Rítmica. Una Exploración de la Transgresión del Orden de Género en el Deporte}

Joaquín Piedra

Universidad de Sevilla

\section{Resumen}

El dominio patriarcal en nuestra sociedad occidental ha oprimido no solo a las mujeres, también ha marginado a muchos hombres que no cumplían con los patrones de la masculinidad hegemónica. El objetivo de este estudio es conocer y analizar las experiencias de un grupo de chicos que practican gimnasia rítmica, tradicionalmente vinculado a lo femenino. Dentro del paradigma interpretativo, se han realizado ocho entrevistas a gimnastas de entre 8 y 19 años que practican gimnasia rítmica a nivel de competición o de exhibición. Los chicos expresan el apoyo familiar que han tenido al introducirse en un deporte tradicionalmente femenino. Sin embargo, muchos de los gimnastas han experimentado alguna vez insultos o bromas por parte de otros chicos. Por ello, parece importante para el futuro trabajar con las familias buscando derribar estereotipos y prejuicios ante esta disciplina masculina. Igualmente se debe promover la gimnasia rítmica entre los niños, pues una mayor presencia de chicos en los clubes facilitaría su acomodo entre las chicas.

Palabras clave: masculinidad, identidad de género, gimnasia, juventud 


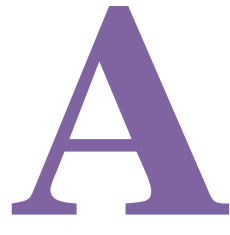

ll over history, some practises (fighting, hunting, leadership, sport...) have been left for the masculine group. Conversely, women were left for other less aggressive activities, more social and linked to the artistic component. Hence, the distribution of roles and tasks has been clearly separated among men and women. Within this rigid social structure, physical activity and sport has been (and is) a modulating context that helps to create and teach gender identities (masculine and feminine) as natural and complementary among them. This construction of identities has provoked the construction of body values linked to the masculine and feminine component (Barbero, 2003). In this social order, masculinity has been built in opposition to femininity, and femininity is built in opposition to masculinity (Connell, 1995). Thus, the physical culture of our society has historically shaped some boundaries for boys and girls (Lamont \& Molnár, 2002). This dual visible separation has made many boys and girls who do not feel identified with the patterns that are established for their body and behaviour model (masculine or feminine) be in a situation of inner fight between what they are and what they have to be, which has enabled the perpetuation of masculine-privilege dynamics (Steinfeldt, Miller, \& David, 2016).

For men, masculinity has been traditionally built on the basis of a model of an aggressive, competitive, heterosexual and strong man. The boys who comply with the masculine stereotype gain more social capital and reach a dominating position in comparison with other boys who do not feel identified or act in a different way from those stereotypes, being separated (Silva, 2013). This position of superiority was named hegemonic masculinity (Connell, 1995; Connell \& Messerschmidt, 2005) and increases the pressure among the rest of boys for imitating it. This dominance situation is accepted and reproduced by an important part of society. The other ways of developing and understanding masculinity are dominated by this hegemonic view, discriminating those men who run away from the "normal" situation. Sport, with its male-centred and heteronormative view, contributes to enhance and promote, in many cases in a subtle and hidden way, this social dominance, increasing even more the boundaries for boys.

Fortunately, the interest towards the situation of masculinities in sport has an extensive academic history. From its beginning, three decades ago, 
research has focused on understanding better the development of the masculine identity within sport speech and practise (Messner, 1992; Messner \& Sabo, 1990; Pronger, 1990). Nowadays, the studies on masculinity in sport remark the clear function of competitive sport as an oppression tool for many boys (Tischler \& McCaughtry, 2011), isolating other types of non-hegemonic masculinities (Atencio \& Koca, 2011; Parker \& Curtner-Smith, 2012; Silva, Botelho-Gomes, \& Goellner, 2012). Within the different forms of oppression and aggression used, homophobia is a recurring one. Nonetheless, as Morrow and Gill (2003) or Silva et al. (2012) state, the relationship between masculinity and sexual orientation cannot be denied, being homosexuality, in many cases, the boundary of accepted masculinity.

This hegemonic masculinity variable made Anderson (2009) pose the concept of homohysteria (the fear of being homosexualised) and build, around it, the theory of inclusive masculinity. According to this theory, cultures can be classified depending on the existing homohysteria levels. A society with a high level of homohysteria makes boys avoid any association with homosexuality, which relegates them to the origin of masculinity hierarchy. Heterosexuality is never challenged in a homophobic atmosphere. Anderson notes that, among men, homohysteria is typically manifested by fleeing from feminized behavior, including physical contact with other men or showing signs of affection and emotion, while also maintaining homophobic discourse. Homohysteria helps to explain how gendered patterns of behavior - both within and outside of sport settings play out in relation to shifting levels of social homophobia, particularly highlighting how homophobia can affect the behavior of individuals who may not necessarily be homophobic themselves.

The scarce studies in Spain in the sport context (Piedra, 2015) prove the different possibilities, from a clear and overwhelming rejection to an acceptance of gays and lesbians. However, in general Spanish culture show evidence of pseudo-inclusivity, and it can be identified as a "politically correct" departure from the rejection of sexual diversity (and thus the negative implication of "being homophobic") alongside a concurrent refusal of acceptance of sexual minorities, shaped perhaps by the heteronormativity of Spain's traditionally conservative political and religious history (Piedra, García-Pérez, \& Channon, 2017). This points out that our society is behind 
if we compare it to others in which there is an atmosphere of increased tolerance. In American and British societies tolerance and inclusivity appear to be deeply rooted, but heteronormativity and heterosexism still persist (Anderson \& McCormack, 2016).

In this situation, in Spain those male athletes who do not comply with the valid characteristics have several options. They can adapt themselves to this masculinity orthodoxy, denying themselves, or they can give up sport, looking for other less hostile activities with their true masculine identity. Fortunately, there is a third group of boys who decide to cross boundaries from the established patriarchal system, showing publicly their heterodox masculinity or looking for sport practises that do not match their gender (Barker-Ruchti, Grahn, \& Lindgren, 2016). This is the case of boys who decide to practise rhythmic gymnastics since this is one of the few sports that tradition has kept for women in an exclusive way, Burstyn (1999) regards them as hyperfeminine. Accordingly, it fosters the features that society considers feminine: flexibility, coordination, balance, thinness and emotions (Hargreaves, 1994; Krane, Choi, Baird, Aimar, \& Kauer, 2004). The boys who decide to practise this sport withstand the "norm" in a double way: they show an identity far from hegemonic masculinity and they do it in a traditionally feminine sport. As Chimot and Louveau (2010) remark, a boy who practises rhythmic gymnastics needs to dare a disputing conflict: he needs to reach a good level of feminine technical expression that is traditionally identified with rhythmic gymnastics, and he also needs to keep his identity as a "true man".

As we have seen, rhythmic gymnastics (formerly known as modern gymnastics) was born as an attempt to highlight traditionally feminine skills. In spite of that, some boys have become part of this scope. Some federations of countries as France or Japan recognise the masculine competition. In Spain, masculine participation was regulated from 2005 in the open category, and the masculine category was recognised as such in 2009, only in its individual category. All in all, masculine participation in Spain, as in other countries, is still a minority, being $1 \%$ of male federated gymnasts. Stereotypes, prejudices and tradition are still the main barriers for the development of this discipline among boys.

This paper uses the context of the Spanish competitive rhythmic gymnastics, which exists in both male and female formats as an entry point 
into these issues. Specifically, it explores what experiences male participants have and how currently masculinities 'do' in what is seen as a 'feminized' arena, and the implications this throws up for practice, policy and future research.

\section{Method}

With the aim of understanding the experience of the boys who practise rhythmic gymnastics and the way that they develop and explain their masculine identity, we use, within the interpretative paradigm, an approach of symbolic interactionism. Symbolic interactionism helps to illuminate how human beings define their experiences and give meaning to their identities, behaviors, realities, and social interactions (Hewitt \& Shulman, 2011). Because of that, eight semi-structured interviews have been done between March 2016 and May 2016 with a length that range from 25 up to 40 minutes.

The sample is made by eight boys whose age range from 8 to 19 years old, from few months to 7 years of experience in rhythmic gymnastics. The reduced size of this sample is due to the limited presence of boys who practise this discipline, there were only 49 masculine licences in our country in 2015. Furthermore, the sample is varied since there are competitive gymnasts and amateurs (belonging to clubs). All gymnasts study and train at the same time. Similarly, the sample has a wide range of experiences, the most veteran individual competes in senior category in the Spanish National Championship and the youngest ones are in the initial competitive levels.

The sampling technique was convenience (Sparkes \& Smith, 2014) through the different clubs who have male gymnasts. After contacting the clubs, the project was presented to families, asking for their agreement with the interview. After the authorization and signature of the parents' informed consent, a date was fixed for the interview. The place of the interview was chosen by the parents, in the club facilities or in their own houses. For its development, the research project has been passed by the Ethical Research Committee of "Junta de Andalucía". The gymnasts are given pseudonyms in the analysis and interview questions are italicized in order to ease its reading. 
The interviews were recorded and transcribed literally for its later analysis with the support of the computer program Atlas.ti. Data analysis was conducted using thematic analysis (Braun \& Clarke, 2006). This is an analytic method in qualitative research for identifying, analyzing and reporting themes across a data-set. Themes were selected if they captured something important about the research question. An inductive and deductive approach, where themes are identified producing descriptions and explanations of particular phenomena (Sparkes \& Smith, 2014).

\section{'Well, Open your Legs': Boys' Experiences with Rhythmic Gymnastics}

Pleasure, interests and motivations are developed at a great extent in childhood and adolescence. In this evolution, familiar environment as well as acquaintances both play a crucial part to promote or hinder some interests. In the case of sport, success is clearly related to athletes' familiar support (Chimot \& Louveau, 2010; Coté, 1999). This has been reflected by most of the interviewed boys, who allude to their parents' support when they began to practise rhythmical gymnastics.

Quite well, I was fully supported because they thought it was a good idea and it was a boy sport, it would be less habitual and it could be exciting then. (Pablo, 11 years old)

Well, they said: “it is ok, if you like it you can try and if you don't like it after some time you can always quit”. (Julián, 13 years old)

I was supported from the very first moment and I have had no problem at all. (Mario, 19 years old)

This familiar support (mainly mothers) has led boys not only to begin practising this discipline, but in most of the cases it implies some trips to competitions or exhibitions and families are the ones who bear these costs. Expenses are sometimes high since the athletic wear is especially expensive. Nonetheless, parents' support has not always been parallel to that of brothers or other relatives, especially male elder ones (Chimot \& Louveau, 2010). Even though these initial doubts are acknowledged, these are later transformed into support. 


\section{MCS - Masculinities and Social Change, 6(3) 295}

My brother was a bit surprised but he then understood me. (Pablo, 11 years old)

At the beginning, they were surprised and laughed, 'where are you going?' because it was a girl sport and you are a boy in rhythmic gymnastics ... but, after some years, they have seen that I am successful and they take it seriously... (Mario, 19 years old)

The same happens with friends. The close circle, initially surprised, has shown their support.

Some people find weird me doing that but, in the end, there has been no problem at all. (Julián, 13 years old)

I usually get on well with everybody, so I have had no problem at all. (Carlos, 18 years old)

They did not say anything, the only thing was "do you practise rhythmic gymnastics?" My answer is yes and they say "well, open your legs" (Antonio, 11 years old)

However, it is also true that many gymnasts have been insulted or mocked by other boys. Miguel (11 years old) and Esteban ( 8 years old) admit that some boys of their age, at school or in their area, made remarks because of practising rhythmic gymnastics.

These stigmatization situations are frequent for boys who participate in other 'feminine' activities as Bemiller (2005) points out in her study with male cheers. This sport structure, as limited for boys who decide to practise traditionally feminine sport activities, show the still existing power of patriarchy in our society, which in many cases challenges these boys' sexuality because of running away from the standard. In most of the cases, boys confirm these experiences in their lives and, in the case of Julián (13 years old), pressure was so high that he decided to quit for some time.

My friends, my school friends, they were a bit surprised and it was weird for them so they didn't accept it [...] so I, with my girlfriend, did some gymnastics in the courtyard, they started to talk about me when I was not with them. (Pablo, 11 years old) 
Had you thought of give up?

Sometimes I did, but then I thought, "Well, what do I care what these guys say?" As a ... [Laughs] ... I do not care. (Julián, 13 years old)

However, these experiences that have affected them, more or less, are not as frequent when we refer to their beginnings in the club. Joining an already created club is a difficult experience, especially when you are the only boy among so many girls. Experiences told by boys are, in all cases, positive and new circles of girlfriends and teammates were created for them. In the few cases in which the initial reception was more sceptical, it would have been easier if the club had had previous experience with other boys, as in the case of some of the visited clubs.

Have you ever felt alone?

Sometimes I have. When we were asked to create groups or pairs, there was nobody who wanted to be with me and I was alone. But, after some time, some girls became my friends and at the end we were together. (Julián, 13 years old)

Hence, the social, economic or institutional difficulties do not mean a reduction of their motivation. The case of Julián (13 years old) can be meaningful since he gave up this practise for a year because of the other boys' remarks and insults. Because of his family support, he began to practise it again and had to fight against the regional federation to enter competitions, collecting signatures and putting pressure so that he was finally accepted.

...when they [regional federation] let us compete they did it because people protested. Not because they said, "Well, here's a guy who wants to do it, so we are going to leave him". No, but because my mother, my friends and all protested so that I could do it, and if not, probably I still could not... (Julián, 13 years old)

In spite of their youth, all these experiences indicate a strong personality and character in all the boys who have been interviewed, together with an unconditional support on behalf of their parents. 


\section{'That's Typical from Girls': Masculine Identity among Gymnasts}

When we go deeper into the subject of identity, nearly all of the interviewed boys admit that rhythmic gymnastics is a girl's sport and they assume it as well as society does. In fact, many boys emphasize the reduced adaptation of the Scoring Code regarding expressivity, choreography and dance to masculine features. In that sense, Bozanic and Miletic's study (2011) on the technical differences among boys and girls highlights the lack of expressivity among boys. This is so noticeable that even boys themselves admit that they do not want to do those movements because they are 'typical from girls', assuming that by doing them they could be identified as girls.

Are those movements always the same for boys and always the same for girls? Are they common, the same for both or do you do different things?

Different things

Would you like to do the same than girls do? Or don't you like those movements?

[Refuses]

Don't you like them? And could you do them?

No, I wouldn't

Wouldn't you like to do them?

No

But could you do it with your body, right?

But I wouldn't

Why not?

Because it's typical from a girl (Vicente, 10 years old)

This problem is not exclusive from rhythmic gymnastics or boys. Krane's study (2001) with women athletes points out that patriarchal and heterosexist society privileges traditional forms of femininity over those which challenge the standards. For that reason, it seems logical that boys assume as improper for them some activities that are socially classified as for girls and boys want to move away from that femininity (Anderson, 2005a). As Priyadharshini and Pressland (2016) point out, tradition, sport structure, social pressure and gender status of some sports are in many cases an obstacle for the change of mind. Doing the opposite could mean discredit, mockery or rejection from an important part of society. 
Nonetheless, this idea contradicts the conclusions reached by Peterson and Anderson (2012) in their studies with male dancers. In this work, researchers indicate that participating in 'feminine' activities is something frequent and it is no longer a subversive activity.

In spite of the fact that everyone recognises that rhythmic gymnastics is less masculine, they all believe that there are different forms of masculinity in which they can fit. For that reason, they value their experience in this discipline as positive, they want to keep practising it and improving. In this sense, Anderson's work (2005b) in another 'feminized' activity as cheerleading indicates that there are two forms of masculinity, a more inclusive one and a more orthodox one which can include different forms of masculinity. This situation is somehow projected by participant boys when differentiating sport from masculine identities.

Yes, it [rhythmic gymnastics] is scarcely masculine but this does not make the person who practises it scarcely masculine. (Carlos, 18 years old)

How would you describe a man, a very masculine boy?

For example, someone who pretends to be cool and strong would join a football team even though he doesn't like it. He would do so just to prove that playing football means being a hard boy. This has got nothing to do because someone who practises rhythmic gymnastics can be harder and more masculine than him. Nothing to do with the sport that he likes. (Pablo, 11 years old)

There are different opinions then while they all recognise themselves as masculine. There are still some boys who have rooted some ideas related to closed roles 'boyish' or 'girlish' preventing both groups from choosing freely those actions, tasks and behaviors that are not typical from their gender.

\section{Conclusions}

Through various interviews we have explored the experiences of boys who practise rhythmic gymnastics, as well as the influences that those experiences have in the construction of their identities. Results show a firm motivation of these boys towards practising a sport that they admit being 
feminine but they feel strongly attracted by. This determination adds to the parents' support to their children. This support does not only imply accepting their children's decision but also encouraging them to continue when they are criticised and supporting them when they need help from institutions.

The families configuration and their view of sport are determining in transmitting the choice of activities of their children, transmitting an intergenerational habitus within these families (McDonald, Rodger, Ziviani, Jenkins, Batch, \& Jones, 2004; Quarmby, 2010). Normally, in the face of more artistic and emotional practices, boys feel closer to their mothers, than boys who do traditionally male sports who are more attached to their fathers (Mennesson, Bertrand, \& Court, 2017). Accordingly, it is important to work with families to eliminate stereotypes and prejudices towards this masculine discipline in the future. This task must be focused on men (parents or brothers) since they usually show rejection, by that clearly rupture the transmission of the paternal sports capital.

Regarding the construction of masculine identity within rhythmic gymnastics, boys show a contradiction in their own statements. While they admit that rhythmic gymnastics is a feminine discipline and they consider themselves masculine, they claim that doing some things would imply (even for themselves) being feminine. There are still limitations and prejudices for boys and girls since some actions and roles are perpetuated as typical from boys or girls.

Finally, we should urge state or regional gymnastics federations to help boys in rhythmic gymnastics. The lack of competitions, the scarce number of clubs with masculine presence and the lack of publicity campaigns contribute to making the spread of this sport among boys even more difficult. We believe, and this is confirmed by Kamberidou, Tsopani, Dallas and Patsantaras's study (2009), that there is a majority of people who support boy participation in rhythmic gymnastics competitions. However, the tradition supported by a minority avoids the acceptance of the presence of boys and it still subjugates many boys from a practise free of prejudices. 
300 Piedra-Masculinity and rhythmic gymnastics

\section{References}

Anderson, E. (2005a). In the game: Gay athletes and the cult of masculinity. Albany, NY: SUNY Press.

Anderson, E. (2005b). Orthodox and inclusive masculinity: Competing masculinities among heterosexual men in a feminized terrain. Sociological Perspectives, 48, 337-355. doi:10.1525/sop.2005.48.3.337

Anderson, E. (2009). Inclusive masculinity: the changing nature of masculinities. New York, NY: Routledge.

Anderson, E., \& McCormack, M. (2016). Inclusive masculinity theory: Overview, reflection and refinement. Journal of Gender Studies, Online First, 1-15. doi:10.1080/09589236.2016.1245605

Atencio, M., \& Koca, C. (2011). Gendered communities of practice and the construction of masculinities in Turkish physical education. Gender and Education, 23, 59-72. Retrieved from http://www.tandfonline.com/doi/abs/10.1080/09540250903519444

Barbero, J.I. (2003). La educación física y el deporte como dispositivos normalizadores de la heterosexualidad. [Physical education and sports as normalizing mechanisms of heterosexuality]. In O. Guasch and O. Viñuales (eds.), Sexualidades: diversidad y control social (pp. 355-377). Barcelona: Bellaterra.

Barker-Ruchti, N., Grahn, K., \& Lindgren E.C. (2016). Shifting, crossing and transforming gender boundaries in physical cultures. Sport in Society, 19, 615-625. doi:10.1080/17430437.2015.1073942

Bemiller, M. (2005). Men who cheers. Sociological Focus, 38, 205-222. doi:10.1080/00380237.2005.10571266

Bozanic, A., \& Miletic, D. (2011). Differences between the sexes in technical mastery of rhythmic gymnastics. Journal of Sports Sciences, 29, 337-343. doi:10.1080/02640414.2010.529453

Braun, V., \& Clarke, V. (2006). Using thematic analysis in psychology. Qualitative Research in Psychology, 3(2), 77-101. Retrieved from http://www.tandfonline.com/doi/abs/10.1191/1478088706qp063oa

Burstyn, V. (1999). The rites of men. Manhood, politics, and the culture of sport. Toronto: University of Toronto Press. 
Chimot, C., \& Louveau, C. (2010). Becoming a man while playing a female sport: The construction of masculine identity in boys doing rhythmic gymnastics. International Review for the Sociology of Sport, 45(4), 436-456. doi:10.1177/1012690210373844

Connell, R.W. (1995). Masculinities. Cambridge, UK: Polity Press.

Connell, R.W., \& Messerschmidt, J.W. (2005). Hegemonic Masculinity:

Rethinking the concept. Gender \& Society, 19(6), 829-859.

doi:10.1177/0891243205278639

Côté, J. (1999). The influence of the family in the development of talent in sport. The Sport Psychologist, 13(4), 395-417.

doi:10.1123/tsp.13.4.395

Hargreaves, J. (1994). Sporting females: critical issues in the history and sociology of women's sports. London, UK: Routledge.

Hewitt, J.P., \& Shulman, D. (2011). Self and society: A symbolic interactionist social psychology (11th ed.). Needham Heights, MA: Allyn and Bacon

Kamberidou, I., Tsopani, D., Dallas, G., \& Patsantaras, N. (2009). A question of identity and equality in sports: men's participation in men's rhythmic gymnastics. Nebula 6.4 a Journal of Multidisciplinary Scholarship Australia, 6, 220-237. Retrieved from http://www.nobleworld.biz/images/Kamberidou_et_al.pdf

Krane, V. (2001). We Can Be Athletic and Feminine, But Do We Want To? Challenging Hegemonic Femininity in Women's Sport. Quest, 53(1), 115-133. doi:10.1080/00336297.2001.10491733

Krane, V., Choi, P., Baird, S.M., Aimar, C.M., \& Kauer, K.J. (2004).

Living the Paradox: female athletes negotiate femininity and muscularity. Sex Roles, 50(5-6), 315-329.

doi:10.1023/B:SERS.00000188

Lamont, M., \& Molnár, V. (2002). The study of boundaries in the social sciences. Annual Review of Sociology, 28,167-195. doi:10.1146/annurev.soc.28.110601.141107

McDonald, D., Rodger, S., Ziviani, J., Jenkins, D., Batch, J., \& Jones, J. (2004). Physical activity as a dimension of family life for lower primary school children. Sport, Education and Society, 9(3), 307325. doi:10.1080/13573320412331302412 
Mennesson, C., Bertrand, J., \& Court, M. (2017). Boys who don't like sports: family lifestyle and transmission of dispositions. Sport, Education and Society, Online First, 1-14. doi:10.1080/13573322.2017.1361397

Messner, M.A. (1992). Power at play: sports and the problem of masculinity. Boston, MA: Beacon Press.

Messner, M.A., \& Sabo, D.F. (1990). Sport, Men and the Gender Order: Critical Feminist Perspectives. Champaign, IL: Human Kinetics Press.

Morrow, R.G., \& Gill, D.L. (2003). Perceptions of homophobia and heterosexism in Physical Education. Research Quarterly for Exercise and Sport, 74(2), 205-214. doi:10.1080/02701367.2003.10609082

Peterson, G.T., \& Anderson, E. (2012). The Performance of Softer Masculinities on the University Dance Floor. Journal of Men's Studies, 20(1), 3-15. doi:10.3149/jms.2001.3

Pronger, B. (1990). The arena of masculinity: sports, homosexuality, and the meaning of the sex. New York: St. Martin's Press.

Parker, M.B., \& Curtner-Smith, M.D. (2012). Sport education: a panacea for hegemonic masculinity in physical education or more of the same? Sport, Education and Society, 17(4), 479-496. doi:10.1080/13573322.2011.608945

Piedra, J. (2015). Gays y lesbianas en el deporte: discurso de jóvenes universitarios españoles en torno a su aceptación. [Gays and lesbians in sport: University students' speech about their acceptance]. Movimento, 21(4), 1067-1081. Retrieved from http://www.redalyc.org/html/1153/115343227017/

Piedra, J., García-Pérez, R., \& Channon, A. (2017). Between Homohysteria and Inclusivity: Tolerance towards sexual diversity in sport. Sexuality \& Culture, Online First, 1-22. doi:10.1007/s12119-017-9434-x

Priyadharshini, E., \& Pressland, A. (2016). Doing femininities and masculinities in a 'feminized' sporting arena: the case of mixed-sex cheerleading. Sport in Society, 19(8-9), 1234-1248.

doi:10.1080/17430437.2015.1096253

Quarmby, T., \& Dagkas, S. (2010). Children's engagement in leisure time physical activity: Exploring family structure as a determinant. Leisure Studies, 29(1), 53-66. doi:10.1080/02614360903242560 
Silva, P. (2013). Construyendo el puzle de la masculinidad en la educación física portuguesa" [Building the puzzles of masculinities in Portuguese PE]. In J. Piedra (ed.), Géneros, Masculinidades y Diversidad (pp. 133-159). Barcelona: Octaedro.

Silva, P., Botelho-Gomes, P., \& Goellner, S.V. (2012). Masculinities and sport: the emphasis on hegemonic masculinity in Portuguese physical education classes. International Journal of Qualitative Studies in Education, 25(3), 269-291. doi:10.1080/09518398.2010.529846

Sparkes, A., \& Smith, B. (2014). Qualitative research methods in sport, exercise and health. London, UK: Routledge.

Steinfeldt, J.A., Miller, I.K., \& David, J.L. (2016). Masculinities in sport: Incorporating heterogeneity into hegemony. In Y.J. Wong, and S.R. Wester (eds.), APA Handbook of Men and Masculinities (pp. 659681). Washington, DC: APA.

Tischler, A., \& McCaughtry, N. (2011). PE is not for me: when boys' masculinities are threatened. Research Quarterly for Exercise and Sport, 82(1), 37-48. doi:10.1080/02701367.2011.10599720

Joaquín Piedra, Department of Physical Education and Sports, Universidad de Sevilla, Seville, Spain

Contact Address: Direct correspondence to Joaquín Piedra, Department of Physical Education and Sports, Universidad de Sevilla, C/ Pirotecnia, s/n, 41013 Sevilla, Spain, email: jpiedra@us.es 
Instructions for authors, subscriptions and further details:

\section{http://mcs.hipatiapress.com}

\section{Misogyny Online. A Short (and Brutish) History}

Beatriz Villarejo $^{1}$

1) Universidad de Deusto, Spain

Date of publication: October $21^{\text {th }}, 2017$

Edition period: October 2017-June 2018

To cite this article: Villarejo, B. (2017). Misogyny Online. A Short (and Brutish) History [Review of the book]. Masculinities and Social Change 6(3), 304-305. doi:10.17583/MCS.2017.3033

To link this article: http://dx.doi.org/10.4471/MCS.2017.3033

\section{PLEASE SCROLL DOWN FOR ARTICLE}

The terms and conditions of use are related to the Open Journal System and to Creative Commons Attribution License (CC-BY). 


\section{Reviews (I)}

Jane, E. (2017). Misogyny Online A Short (and Brutish) History. London: SAGE Swifts. ISBN: 978-1473916005

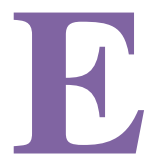

mma Jane empieza el libro utilizando una expresión que muchas mujeres se están encontrando cuando acceden al ciberespacio: Get the Fuck Out. Un ejemplo de la existencia de una misoginia que a menudo no está encontrando rechazo social para poder ser erradicada. De hecho, Jane acuña el término genderhate para referirse a como las mujeres sufren situaciones de odio y acoso en las redes. Uno de los ejemplos que se utiliza en este caso es la comunidad de juegos virtuales GamerGaters, que para la autora es especialmente misógina y que en ocasiones promueve actitudes violentas hacia la mujer. Esta comunidad tiene como principal objetivo crear una campaña contra el feminismo moderno a través del acoso.

En el primer capítulo 1 del libro titulado The rise of Rapeglish se hace un breve repaso a cómo se ha ido extendiendo el odio hacia la mujer en el ciberespacio desde la década de los noventa a hasta la actualidad. Para ello se pone de manifiesto uno de los datos más relevantes que recoge un informe de las Naciones Unidas en el que se expone que alrededor del $73 \%$ de las mujeres en todo el mundo están expuestas a algún tipo de violencia online. De la multitud de ejemplos que Jane expone en su libro, destacamos a continuación una de los comentarios con los que se encontró en los 90': What is your agenda? Girl power? To hate men? Or just being your stupid self. You should have a good arse fuck lasting two hours every day.

El capítulo 2, What it is so, se centra en la dimensión etiológica sobre el odio hacia la mujer que se articula en Internet. En este sentido, se especifica que se trata de un odio estructural que influencia el discurso también en los espacios virtuales. La autora expone dos razones por las cuales este odio se

2017 Hipatia Press

ISSN: 2014-3605

DOI: $10.17583 / \mathrm{MCS} .2017 .3033$ 
perpetúa: 1) por la necesidad de algunos hombres de mantener sus privilegios a escala social, y por ende, en internet; 2) y por la legislación laxa y la débil normativa existente en el espacio virtual sobre los ataques y el acoso.

En el capítulo 3, Hitting Home, se exponen las consecuencias de estas prácticas misóginas en internet aprovechando la literatura científica sobre filosofía. En este sentido se explica cómo se utiliza la coerción para argumentar por qué las mujeres se van de internet para evitar acosos, y como esta acción se fundamenta en una elección racional y no en una decisión libre. También se subraya cómo se articulan prácticas misóginas que intentan silenciar la voz de las mujeres, sobretodo de los colectivos feministas, en dichos espacios virtuales.

En los capítulos 4 y 5 se expone la forma en que las mujeres están constantemente siendo culpadas en internet por sus actuaciones mientras que los hombres son eximidos y excusados la mayoría de las veces. De hecho, la autora argumenta que la anteriormente mencionada culpabilización de la mujer es un mecanismo también para excusar a los hombres.

Finalmente, en las conclusiones se presentan algunas propuestas de mejora para acabar con la problemática sobre la que versa el libro. Sobre todo, la autora afirma que la misoginia on-line no puede esperar que se finalice la off-line, debe ser una actuación paralela desde un posicionamiento feminista firme.

Beatriz Villarejo, Universidad de Deusto. beatriz.villarejo@deusto.es 
Instructions for authors, subscriptions and further details:

\section{http://mcs.hipatiapress.com}

\section{Masculinity and Aspiration in the Era of Neoliberal Education}

Garazi Lopez de Aguileta ${ }^{1}$

1) Universidad de Barcelona, Spain

Date of publication: October $21^{\text {th }}, 2017$

Edition period: October 2017-June 2018

To cite this article: Lopez de Aguileta, G. (2017). Masculinity and Aspiration in the Era of Neoliberal Education [Review of the book]. Masculinities and Social Change 6(3), 306-307. doi:

10.17583/MCS.2017.3019

To link this article: http://dx.doi.org/10.4471/MCS.2017.3019

PLEASE SCROLL DOWN FOR ARTICLE

The terms and conditions of use are related to the Open Journal System and to Creative Commons Attribution License (CC-BY). 


\section{Reviews (II)}

Stahl, G., Derrick-Nelson, J., \& Wallace, O. (Eds.). (2017). Masculinity and Aspiration in the Era of Neoliberal Education. New York: Routledge. ISBN: 978-1-138-12303-8

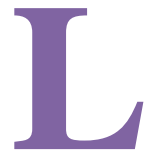

a crisis financiera de los últimos años ha hecho replantear muchos análisis sobre el devenir del neoliberalismo en occidente. El libro que presentamos en esta review es un ejemplo de ello pero en este caso a través del estudio de las aspiraciones educativas de los varones. De este modo, el libro empieza planteando un objetivo claro e importante en este contexto: entender qué factores sociales, culturales y especiales han influenciando la construcción de la masculinidad.

Los editores del libro nos dejan claro que estamos ante un libro que podríamos situar en la literatura reciente de sociología de las masculinidades que adopta cierta importancia en la década de los 90 con autores relevantes como Kimmel y Mac an Ghaill. De hecho, en los últimos años, pero también en sus inicios, los men's studies han tenido un fuerte lazo con la sociología realizando estudios trascendentes sobre las identidades de género y los procesos de construcción de la masculinidad.

El libro, a partir de diferentes capítulos, plantea qué efectos está teniendo el neoliberalismo en el ámbito educativo y los discursos sobre las aspiraciones y su repercusión en los chicos escolarizados. En este sentido, en la introducción se pone de manifiesto que las políticas de austeridad que se han implementado desde diferentes países han influenciado los comportamientos en diferentes ámbitos de socialización, como por ejemplo los centros escolares. En este sentido, se observa que ha aumentado la competitividad en las relaciones sociales y ello ha tenido un efecto directo en la reproducción de las imágenes tradicionales de masculinidad. Por ejemplo,

2017 Hipatia Press

ISSN: 2014-3605

DOI: $10.17583 / \mathrm{MCS} .2017 .3019$ 
en el libro se especifica que la existencia del "achievement ideology" es un planteamiento que fortalece la reproducción de estas imágenes.

A lo largo de los diferentes capítulos se ilustran diferentes exámenes sobre como la institución escolar influencia las aspiraciones de los niños y chicos. Por ejemplo, hay un grupo de capítulos que se centran en el Reino Unido poniendo de manifiesto la dificultad de este país en promover la movilidad social y los fallidos esfuerzos de las políticas sociales y educativas para conseguirlo. Respecto a la construcción de la masculinidad, los autores expresan la persistencia de un machismo en la socialización de género que perpetúa unos esquemas de género muy arcaicos.

En otros tres capítulos se realiza el mismo análisis por en este caso en Estados Unidos al que se critica ferozmente por convertirse en la bandera principal del neoliberalismo. De hecho, se ironiza en el libro sobre el llamado sueño americano porque se percibe como un discurso que no favorece una socialización diferente. En estos capítulos también se habla de las aspiraciones de los chicos que provienen de familias latinas o afroamericanas. Se insiste en la diferenciación existente de estos colectivos con el colectivo hegemónico.

Por último, quería señalar los análisis efectuados sobre la masculinidad y las aspiraciones en países como China y Australia. De hecho, resulta interesante e innovador el enfoque expuesto sobre el gigante asiático ya que en él se subraya la permanencia de un discurso de los "failing boys" muy perjudicial para los chicos de este país. De alguna forma, legitima la existencia de "chicos de segunda" que no poseen las mismas expectativas ni consiguen los mismos objetivos que los chicos que obtienen buenos resultados académicos.

Garazi López de Aguileta, Universidad de Barcelona. garazi.jaussi@gmail.com 